\title{
Modelling chemical signalling cascades as stochastic reaction diffusion systems
}

\author{
$\mathrm{PhD}$ Thesis
}

in partial fulfilment of the requirements for the degree Doctor of Philosophy $(\mathrm{PhD}) / \mathrm{Dr}$. rer. nat. in the Neuroscience Program at the Georg August University Göttingen,

Faculty of Biology

submitted by

\section{Garrit Jentsch}

born in

Henstedt-Ulzburg 


\section{Contents}

1 Introductus $\quad 5$

1.1 Chemical signalling . . . . . . . . . . . . . . . . 5

1.2 A short history of Monte Carlo Methods in chemical signalling . . . . . . 8

1.3 Overview and objective of this work . . . . . . . . . . . . . . . . . 11

2 Main Theme: Stochastic methods in signalling 13

2.1 Introducing the Chemical Master Equation . . . . . . . . . . . . . . . . 13

2.2 Operator Descriptions of the Chemical Master Equation: Single Compartment . . . . . . . . . . . . . . . . . . . 17

2.2.1 Functional Integral Representation . . . . . . . . . . . . . . 21

2.2.2 Rate equations and noise induced effects . . . . . . . . . . . . 23

2.2 .3 Noise induced effects . . . . . . . . . . . . . . . . . . 25

2.3 Spatially Inhomogeneous Reaction Systems . . . . . . . . . . . . . . . 28

2.4 Monte Carlo Algorithms . . . . . . . . . . . . . . . . . . . . . . . . . . . 31

2.4.1 Direct Method . . . . . . . . . . . . . . . . . . . 32

2.4.2 Next Subvolume Method . . . . . . . . . . . . . . . . . . . . 34

2.4.3 Logarithmic Classes Method . . . . . . . . . . . . . . . . . . . . 36

3 Variation 1: Comparing the efficiency of the different approaches $\quad 39$

3.1 Introduction . . . . . . . . . . . . . . . . . . . . . . 39

3.2 Comparison . . . . . . . . . . . . . . . . . . . . . . . . 40

3.2.1 Autocatalytic waves . . . . . . . . . . . . . . . . . . . . . 40

3.2.2 Localised spots . . . . . . . . . . . . . . . . . . . . . . . . . . . . . . . . . . . . . . . . . . . . . . . .

3.3 Discussion . . . . . . . . . . . . . . . . . . . . . . . . . . . . 43

3.3.1 Analysis of the different Monte Carlo methods . . . . . . . . . . 43

3.3.2 Improved version of the Logarithmic Classes Method . . . . . . . 45

4 Variation 2: Monte Carlo Simulation of lateral signal propagation $\quad 49$

4.1 Introduction . . . . . . . . . . . . . . . . . . . . . . . . . . . 49

4.2 Lateral signal propagation . . . . . . . . . . . . . . . . . . . . 51

4.2 .1 Experimental finding . . . . . . . . . . . . . . . 51

4.2 .2 Current Model . . . . . . . . . . . . . . . . . . . 53

4.3 Extending the current model . . . . . . . . . . . . . . . . 56

4.3.1 Mathematical modelling . . . . . . . . . . . . 56 
4.3 .2 Computational Modelling . . . . . . . . . . . . . 60

4.4 Molecular crowding . . . . . . . . . . . . . . . . . . . . . . . . . 68

4.4.1 Lateral signal propagation in the presence of molecular crowding . 68

4.4.2 Chemical wave fronts in an inhomogeneous medium . . . . . . . 71

5 Variation 3: Representing space revisited $\quad 77$

5.1 Aim and Background . . . . . . . . . . . . . . . . . . . . 77

5.2 Transformed Grid Monte Carlo . . . . . . . . . . . . . . . . . . . . . . 79

5.2.1 Cylindrical coordinates . . . . . . . . . . . . . . . . . . . . . . . 79

5.2.2 Spherical coordinates . . . . . . . . . . . . . . . . . . 80

5.2.3 Combing different geometries for the modelling of realistic biological structures . . . . . . . . . . . . . . . . . . . . 81

5.2.4 General case . . . . . . . . . . . . . . . . . . . . 85

5.3 Finite Volume Monte Carlo . . . . . . . . . . . . . . . . . . . . . . . . 90

5.4 Eukaryotic Chemotaxis . . . . . . . . . . . . . . . . . . . . . . . . 93

5.4 .1 Gradient Sensing . . . . . . . . . . . . . . . . . . . . . . . . 93

5.4.2 Computational Modelling of Gradient Sensing . . . . . . . . . . 95

5.5 Discussion . . . . . . . . . . . . . . . . . . . . . . 100

6 Coda: Conclusion and outlook 101

6.1 Conclusion . . . . . . . . . . . . . . . . . . . . . . . . . . . . 101

6.2 Outlook . . . . . . . . . . . . . . . . . . . . . . 102

$\begin{array}{ll}\text { Bibliography } & 103\end{array}$ 


\section{Introductus}

in which we learn about the biological basics of chemical signalling cascades and in which we stretch that chemical signalling cascades have to be understood as spatially resolved stochastic processes.

1.1 Chemical signalling ................ 5

1.2 A short history of Monte Carlo Methods in chemical signalling .................. . . 8

1.3 Overview and objective of this work . . . . . . . . . 11

\subsection{Chemical signalling}

A prerequisite for cells to live together in a coordinate manner within a single organism is their ability to communicate with one another [Alberts et al., 1994]. Often, cell-cell communication is achieved with the help of specific extracellular molecules which are synthesised and released by signalling cells. If these substances bind to complementary receptors within their target cells, they are capable of activating so-called signalling cascades. The term signalling cascade, as well as the synonymously used term signal transduction pathway, refers to the intracellular biochemical reactions by which a target cell is capable of responding to external stimuli. Ultimately such a response leads to a change in cellular behaviour which may manifest itself in an altered metabolism, proliferation, or differentiation. Overall, the fact that cellular events are precisely regulated by chemical signal transduction cascades is quite remarkable because signalling cascades are inherently noisy. In general, this sort of noise arises because the local concentrations of the key molecular species involved in a chemical reaction cascade are often very low. In the literature this sort of noise is often referred to as intrinsic noise. However, apart from this intrinsic noise cells also have to cope with extrinsic noise as they normally live in a noisy environment.

Although some exceptions such as the steroid hormone signalling pathway exist, chemical signalling cascades are normally activated by receptor proteins within the plasma membrane of the cell. These receptors act as signal transducers converting the extracellular into an intracellular signal. Basically this transduction step is achieved by means of a conformational change within the receptor which is caused by the binding of a ligand. 
Overall three main classes of signal transduction within cell surface receptors are known to exist. The simplest class comprises receptors linked to ion channels, which open or close their pore upon stimulation. G-Protein coupled receptors, which make up the second class, release their intracellular G-Protein upon a conformational change. This protein can subsequently interact with other intracellular proteins resulting for instance in the amplification of the incoming signal by the production of so-called second messengers. Finally, enzyme linked receptors either exhibit an intrinsic enzyme activity or alternatively are associated with an enzyme. Most commonly these enzymes are kinases that phosphorylate their target molecules if the receptor is in its active state. Generally protein phosphorylation and its counterpart protein dephosphorylation which is mediated by so-called phosphatase molecules, play a prominent role in chemical signalling. Through cycles of phosphorylation and dephosphorylation reactions a signal can be propagated from the cell surface to the nucleus. This is often the destination of a signal transduction pathway, because a sustained change in cellular behaviour can be achieved by a change in gene expression.

Although the interactions within signalling cascades are highly specific, chemical signalling can not be regarded as a linear chain of reactions, where one reaction is simply executed after another until a response is finally triggered. Instead, incoming information is processed and analysed with respect to the internal state of the cell. Overall a signal transduction pathway is therefore better understood as a small part of a much larger signalling network. Furthermore when studying signalling networks, it has become apparent within the past few years that spatial and temporal dynamics play a crucial role in specifying the behavioural response to an external signal [Kholodenko, 2006]. For instance, for the mitogen activated protein kinase (MAPK) cascade it is known that the same repertoire of signalling molecules can give rise to different behaviours. If this signal transduction pathway is transiently stimulated by the epidermal growth factor (EGF), this results in cell proliferation, whereas cell differentiation will be caused by a sustained stimulation of nerve growth factor (NGF) [Murphy et al., 2002].

Investigating how the processing of chemical information is brought about within biological cells is the cornerstone of the emerging field of systems biology [Kirschner, 2005]. With the advance of new experimental techniques such as sequencing, microarray analysis, mass spectrometry, and a wide range of optical methods which allow for the observation of signalling cascades in action, recent years have witnessed a tremendous increase in quantitative data about biological systems. Today, entire genomes have been sequenced, protein structures have been analysed, and metabolic pathways have been characterised. When it comes to chemical signalling, entire wiring diagrams can be drawn showing which constituents of a cascade interact with one another. However, the sheer presence of an overwhelming amount of data does not necessarily imply that we also have a deeper understanding of the processes within a cell. After all, the aim is to understand how different molecular constituents interact in space and time within a dynamic environment in order to reliably regulate the cell's behaviour. This task clearly calls for mathemat- 
ical modelling. Overall, we require that every mathematical model has to account for the noise that is omnipresent in biological systems. Moreover, signalling cascades have to be spatially resolved. Both aspects are obviously neglected when treating chemical signalling as simple deterministic wiring diagrams. However, these requirements make an analytical mathematical treatment of chemical signalling almost impossible. In fact, analytically tractable mathematical models have mostly been proposed and used for the analysis of prototypical motifs found within signalling networks. Common examples include the study of protein phosphorylation cycles and the role played by positive and negative feedback [Tyson et al., 2003]. As opposed to that, the mathematical analysis of real world signal transduction pathways, which one for instance encounters when investigating which disfunctioning of a signalling cascade leads to cancer, is analytically infeasible. Instead, numerical tools have been developed. Today, computer simulations have established themselves as valuable tools for pursuing the aim of understanding the intracellular signalling machinery.

Overall, a multitude of different computational approaches are available when it comes to simulating the temporal dynamics of chemical signalling. The most common approach is based on numerically integrating the differential equations describing the change of the involved molecular concentrations with respect to time. When pursing this approach, one implicitly assumes that molecular concentrations are continuous functions of time. However, this is only valid in the limit of having large numbers of molecules. Its application is bound to fail when dealing with many in vivo processes, for which the total number of molecules of a certain species can become very small within particular spatial regions. If this is the case, stochastic effects have to be taken into account [Rao et al., 2002]. A prominent example is given by the modelling of signalling cascades responsible for the regulation of synaptic efficiency within the dendritic spine. Here the underlying reaction volume is so small that the key regulator proteins will only be present in a few copy numbers. In such a case reactants have to be accounted for by discrete numbers of particles. On this level of description chemical reactions are no longer deterministic in nature but occur stochastically so that their time of occurrence as well as their exact order can no longer be predicted [Bhalla, 2004]. Another example in which stochasticity severely affects the overall behaviour of a biological system is genetic transcription and the subsequent translation of mRNA into protein. Thattai and van Oudenaarden [2001] developed a simple analytic model which showed that the production of proteins in eukaryotic cells has to occur in random bursts. In a subsequent experimental study, they could further shown that the number of proteins produced in each burst as well as the time in between bursts is highly variable and that the exact statistics depend on the involved reaction rates as predicted by their theory [Ozbudak et al., 2002]. Hence, protein concentrations exhibit a great deal of variability such that a population of isogenetic cells can display phenotypic variations [Elowitz et al., 2002]. Clearly such a behaviour can not be explained with a deterministic approach in which the proteins are synthesised with constant given rates.

Furthermore, when modelling chemical signalling, it is also important to spatially resolve 
the medium in which the chemical reactions take place. Even prokaryotes, which are normally modelled as simple homogeneous reaction volumes, are spatially organised [Moraru and Loew, 2005]. For instance, the receptors mediating the chemotactic response in $E$. coli are organised into clusters which ultimately increases the reliability of the response [Sourjik, 2004]. That prokaryotic cells are highly spatially organised is immediately obvious as they contain different compartments which define regions of specialised functionality. Furthermore prokaryotic cells are also spatially organised on the subcompartment scale. Recent years have for example witnessed an increased interests in concentration gradients of molecules involved in chemical signalling [Kholodenko, 2006]. In the context of prokaryotic chemotaxis such gradients are extremely important. It has been shown that the movement of a cell depends on the uneven distribution of some key molecules along the plasma membrane, which is established by the so-called gradient sensing signalling cascade. This reaction diffusion network senses and amplifies an external gradient field and thereby provides the information into which direction the cell has to move [Janetopoulos et al., 2004].

Overall, complex in vivo reaction systems show non-trivial spatial reaction patterns that need to be accounted for in the modelling process. It is therefore highly advisable to use a stochastic simulation method which correctly accounts for the underlying geometry of the reaction medium. In the next section we will therefore briefly resketch the history which lead to the most common stochastic approaches that exist today for simulating cellular regulatory pathways in a stochastic manner.

\subsection{A short history of Monte Carlo Methods in chemical signalling}

Stochastic computational methods are generally referred to as Monte Carlo techniques as they utilise (pseudo) random numbers. The first such method was the Metropolis algorithm which was invented for calculating the free energy of a system of atoms [Metropolis et al., 1953]. As opposed to such a static Monte Carlo simulation method used for the calculation of state variables, the stochastic techniques used when simulating biological reaction systems belong to the overall class of kinetic or dynamic Monte Carlo algorithms [Fichthorn and Weinberg, 1991]. These algorithms are used for simulating the temporal evolution of Markov processes of which the dynamics are known to obey given transition rates. Therefore, these computer methods are applicable to all sorts of different physical problems ranging from spin glasses to polymer dynamics. Due to this almost the same algorithms have been reinvented a couple of times within different areas of application.

When modelling chemical signalling in a stochastic way, a mesoscopic level of description is often useful and appropriate. Reactants are individually accounted for in the sense that the state of a system is completely determined by counting how many particles 
belong to a particular chemical species. The distribution of particles of the different chemical species is changed by chemical reactions, which randomly occur with a given probability. The first two algorithms for simulating such mesoscopic chemical reaction systems were proposed by D. Gillespie in 1976 and are termed the Direct Method and the First Reaction Method [Gillespie, 1976]. These algorithms are capable of simulating chemical reaction systems in which the molecules are homogeneously distributed in space. However, the real breakthrough and application of these algorithm to the field of chemical signalling did not come until the late 1990s, when it was shown that the outcome of signal transduction pathways depends in a nontrivial way on the underlying level of noise within a cell. Consequently questions were asked addressing for instance the problem of how cells can reliably respond to external stimuli, if the underlying signal transduction machinery is subject to noise. Clearly, computational studies became a means for answering these questions and first examples employing the Gillepie algorithm include for instance [Arkin et al., 1998], in which it was shown how cellular decisions are influenced by a stochastic environment.

However, with the application of the Gillespie algorithm to real world problems, two limitations became immediately obvious. First of all, the original algorithms proposed by Gillespie are computationally inefficient as they directly scale with the number of chemical reactions being simulated. This limitation is intensified by the general problem arising within stochastic simulations that the more particles there are within the medium, the more often a reaction channel fires within a certain time interval. This behaviour arises because the reaction rates increase with the number of particles in the system. The second problem of the algorithm lies in its non-consideration of space. This has to be regarded as a major drawback, since for most chemical signalling cascades the shape and size of the underlying reaction volume are of great importance. In fact neglecting space may lead to the violation of the underlying assumption that molecules are in spatial equilibrium.

For the first problem better algorithms such as the Next Reaction Method were proposed. With this algorithm a great gain in computational efficacy could be obtained by using more sophisticated data structures [Gibson and Bruck, 2000]. In most scenarios, this algorithm proved to be a lot faster than the original two algorithms proposed by Gillespie. Another attempt to increase computational efficacy was suggested by Gillespie in 2001, when he proposed the so-called Tau-Leap method [Gillespie, 2001]. Instead of just picking a single reaction and updating the reaction rates after each step, this method works by determining how often a reaction will fire within a given time interval. In order for the algorithm to produce meaningful results, the length of the time interval has to be chosen in such a way that the change in the underlying rates is small compared to some given error. Hence the increase in speed within this method comes with a loss in accuracy.

For the latter problem of representing space within stochastic simulations of chemical reaction systems, different solution strategies have been proposed [Lemerle et al., 2005]. One such strategy abandons the mesoscopic simulation approach all together. Instead, a more detailed description of the underlying system is employed by representing each 
chemical reactant individually meaning that it now has a specific position within the reaction medium associated to it. Spatial dynamics are then accounted for in that particles perform random walks. If two particles encounter each other, a chemical reaction may occur with a certain probability. Within the literature this approach is known as the Brownian dynamics approach. For the first time it has been employed within the MCell environment, which was developed for the modelling of cellular signalling at the synapse [Stiles and Bartol, 2001]. Although MCell could in principle be used for simulating intracellular chemical dynamics, the emphasis clearly lies on extracellular dynamics; i.e. it has mainly been used to model diffusion of ligand molecules within the synaptic cleft as well as for the subsequent interaction of these molecules with ion-channels and transporter molecules at the postsynaptic side. Compared to MCell, Smoldyn is a Brownian dynamics simulator which was developed for the modelling of intracellular chemical signalling [Andrews and Bray, 2004]. It has been successfully applied to the modelling of signalling cascades within bacterial chemotaxis [Lipkow et al., 2005].

However, space can also be easily incorporated within the Gillepie algorithm. For this task the overall reaction volume has to be divided into a set of subvolumes. This has to be done in such a way that the size of the subvolumes is sufficiently small so that the particles will always be uniformly distributed within the boundaries of each subvolume. In case that this so-called well stirred criterion holds true, the Gillepie algorithm can again be used for the simulation of chemical reactions within the subvolumes. In addition one has to account for the diffusive movement of the particles by incorporating jump processes which exchange particles between adjacent subvolumes. So far this approach, which is also referred to as voxelisation in the literature, has been utilised by different groups. [Stundzia and Lumsden, 1996] used a straightforward implementation of the Gillespie algorithm to model the propagation of a calcium wave within a cell. In contrast to that, [Elf and Ehrenberg, 2004] based their algorithm for the modelling of bistable chemical systems on the Next Reaction method.

Another stochastic approach for simulating chemical signalling which for the sake of completeness should be mentioned here is the Stochsim algorithm developed by MortonFirth in 1998 [Morton-Firth and Bray, 1998]. Like the Gillespie algorithm it is capable of modelling chemical signalling in spatially equilibrated systems. However, the way by which this is achieved is somewhat different from the Gillepie method. Within Stochsim molecules are represented as individual data objects and the algorithm works by testing in each time step whether a chemical reaction can occur between a randomly chosen pair of particles. This somewhat different approach comes with the advantage that multistate molecules can be easily represented within Stochsim. However, the fixed time step of the method has to be viewed as a disadvantage, since the algorithm's accuracy and efficacy heavily depend on the choice of the time step. Nevertheless the algorithm was successfully applied to elucidate certain problems within bacterial chemotaxis.

Today several simulation environments exist that allow for the deterministic and stochas- 
tic modelling of chemical signalling. Often these programs are downloadable from the world wide web. For a recent review on non stochastic simulation environments one should consult [Alves et al., 2006]. To mention but a few of the stochastic environments currently available, the Brownian dynamics simulators Smoldyn (http://www.pdn.cam.ac.uk/groups/compcell/Smoldyn.html) [Andrews and Bray, 2004] and MCell (http://www.mcell.cnl.salk.edu) [Stiles and Bartol, 2001] should be mentioned as well as MesoRD (http://mesord.sourceforge.net/) [Hattne et al., 2005], and the SmartCell program package (http://smartcell.embl.de) [Ander et al., 2004] as two examples for mesoscopic stochastic simulation environments.

\subsection{Overview and objective of this work}

Chemical signalling cascades have to be regarded as spatially resolved stochastic processes. Hence they function differently than deterministicly working control circuits. However, this is the picture that is generally suggested by depicting chemical signalling cascades as simple wiring diagrams showing which molecular species interact with one another. Overall, the main goal of this thesis is therefore to develop and apply stochastic spatial methods which allow for a more realistic modelling of chemical signal transduction cascades.

Chapter 2 comprises the fundamentals of modelling reaction diffusion systems in a mesoscopic stochastic fashion. In particular, we will show how a reaction diffusion system can be modelled by a Markovian process which is completely characterised by the so-called reaction diffusion master equation. Furthermore we will present an operator description of this equation. This part of the thesis is based on the works of Cardy and Täuber [1998] who employed field theoretic and renormalisation methods in order to fully describing the effects of noise in simple reaction diffusion systems. We extend their method such that it is possible to describe arbitrary reaction networks and account for diffusion in arbitrary geometries at the same time. Furthermore we show that this formulation allows for a systematic small noise expansion and provides an easy approach for quantifying the effects induced by external noise. Note, that this approach is quite different than a standard Langevin approach which simply adds a noise term to the deterministic rate equation. Finally, we will end that chapter by presenting three different Monte Carlo algorithms which generate trajectories according to the chemical reaction diffusion master equation. Chapter 3 compares the computational efficacy of these three Monte Carlo algorithms. For the comparison we introduce two simple test scenarios, which differ in the extent with which the reactivities vary within the reaction medium. A detailed analysis of the algorithms will explain the difference in the algorithms' performances. Furthermore the analysis leads to an improved version of the logarithmic classes algorithm.

In the fourth chapter we will then investigate whether chemical wave fronts provide a reliable mechanism for propagating a signal over long distances. As a model system we will turn to the EGF receptor system, in which travelling fronts propagate a phosphorylation 
signal across the entire membrane. In particular, we will analytically compare a two dimensional with a three dimensional model. Furthermore we will numerically investigate which effects arise if one accounts for the noise. In this part, we will see that chemical noise can lead to the spontaneous phosphorylation of all the receptors. Finally, we will investigate how spatial noise caused by molecular crowding affects the overall properties of a chemical wave front.

Finally, in Chapter 5 we introduce two new methods with which one can represent the geometry of a reaction medium in a more realistic fashion than it is currently possible in stochastic simulation environments. These two methods are the Transformed Grid Monte Carlo method and the Finite Volume Monte Carlo method. To demonstrate the feasibility of our approach we derive a minimal model of the gradient sensing reaction network in Dictyostelium discoideum and show that the outcome of this signalling cascade depends on the underlying geometry. 


\section{Main Theme: Stochastic methods in signalling}

in which the underlying equation, the reaction diffusion master equation is presented and in which we show how it is employed for simulating chemical signal transduction. Furthermore we will introduce an operator description which is used to demonstrate that noise may lead to counter-intuitive effects which are not foreseen by a deterministic description.

2.1 Introducing the Chemical Master Equation . . . . . . . 13

2.2 Operator Descriptions of the Chemical Master Equation: Single Compartment . . . . . . . . . . . 17

2.2.1 Functional Integral Representation . . . . . . . . . . . 21

2.2.2 Rate equations and noise induced effects . . . . . . . 23

2.2 .3 Noise induced effects . . . . . . . . . . . . . . 25

2.3 Spatially Inhomogeneous Reaction Systems . . . . . . . 28

2.4 Monte Carlo Algorithms . . . . . . . . . . . . . . . . . . 31

2.4.1 Direct Method . . . . . . . . . . . . . . . 32

2.4.2 Next Subvolume Method . . . . . . . . . . . . . . . 34

2.4.3 Logarithmic Classes Method . . . . . . . . . . . . . . . 36

\subsection{Introducing the Chemical Master Equation}

When thinking about chemical signalling in physical terms the picture that immediately comes to mind is that of a reaction diffusion system. For our underlying model of chemical signalling we therefore assume that we have a set of molecules immersed in a solvent which can react with one another. Each particle has its own position and momentum and both solvents and reactants are governed by Newton's laws of motion. In the case of two particles colliding with one another they either perform an elastic collision or they undergo a chemical reaction. However, since the number of reactant molecules is greatly outnumbered by solvent molecules, collisions among solvents and reactants occur much more frequently than collisions among reactants. Hence, when simulating chemical signalling on this level of detail, one would most certainly account only implicitly for the 
solvent molecules in that they cause the reactants to perform a random walk which is correctly described by Brownian motion [Grabert, 1982]. This reasoning leads directly to the Brownian Dynamics approach which was already briefly described in section 1.2. However since the simulation of complex reaction systems according to this method is computationally very expensive as it scales with the number of particles in the system, we will now and for the rest of this thesis turn to a mesoscopic level of description.

If the underlying volume of a chemical reaction system is chosen sufficiently small, so that diffusion is fast enough to abolish spatial inhomogeneities inside the volume between successive reactions, the system is said to be well stirred. From a modelling perspective this means that diffusion does not have to be accounted for. In such a scenario one can describe the state of a biochemical system within the reaction volume $\Omega$ by a vector $\mathbf{n}=\left(n_{1}, \ldots, n_{M}\right)^{T}$, where $n_{i}$ denotes the number of particles of species $A_{i}, i \in 1, \ldots, M$. The system's state is updated whenever a chemical reaction $R_{\alpha}$ $(\alpha \in 1, \ldots, R)$ occurs. Each occurrence of reaction $R_{\alpha}$ changes the state from $\mathbf{n}$ to $\mathbf{n}+\boldsymbol{\Delta}_{\alpha}$. The $\boldsymbol{\Delta}_{\alpha}$ are the stoichiometric coefficients and for later convenience we split the $\boldsymbol{\Delta}_{\alpha}=\boldsymbol{\Delta}_{\alpha}^{+}-\boldsymbol{\Delta}_{\alpha}^{-}$, where the index \pm refers to an increase $(+)$or decrease $(-)$of particle numbers. Hence, the indices refer to the products and educts of a chemical reaction, such that the $\Delta_{\alpha}^{-}$are determined from the stoichiometric coefficients on the left hand side of a reaction and $\Delta_{\alpha}^{+}$from the right hand side. Apart from its stoichiometry a reaction $R_{\alpha}$ is further characterised by its propensity function $\lambda_{\alpha}(\mathbf{n}, t)$. This function is defined in such a way that $\lambda_{\alpha}(\mathbf{n}, t) d t$ gives the probability (to first order in $d t$ ) that reaction $\alpha$ occurs within the infinitesimal time interval $[t, t+d t]$. The probability of more than one reaction occurring in $d t$ is negligible as it is proportional to $d t^{2}$. Furthermore, in the absence of time dependent external fields, $\lambda_{\alpha}(\mathbf{n}, t)$ does not explicitly depend on time. A simple argument to fix the propensities on particle numbers makes use of the kinetic collision theory of reactions. For instance, for the reaction $A+B \rightarrow C$ the propensity function is given by

$$
\lambda\left(n_{A}, n_{B}\right)=k n_{A} n_{B},
$$

whereas the propensity function for the reaction $A+A \rightarrow B$ is given by

$$
\lambda\left(n_{a}\right)=k n_{a}\left(n_{a}-1\right) .
$$

In the two equations above $k$ denotes the second order mesoscopic rate constant of the respective reaction and $n_{a}$ and $n_{b}$ account for the number of particles of the molecular species $A$ and $B$. Overall the probability for the occurrence of a reaction is proportional to the number of distinct reactant pairs that can participate in a reaction. Note that the mesoscopic rate constant $k$ has units of (time) $)^{-1}$. Since second order macroscopic rate constants have the dimension (moles $/$ litre $)^{-1}(\text { time })^{-1}$, the relation between the two constants is given by

$$
k_{\text {macroscopic }} \sim k_{\text {mesoscopic }} \times A \times \Omega \times C,
$$


where $A$ is Avogadro's number, $\Omega$ denotes the volume of the reaction medium, and $C$ is a dimensionless constant. For the case that the reaction involves reactants of two different chemical species $C=1$, whereas $C=1 / 2$, if the reaction involves two molecules of the same type.

Starting from kinetic theory, Gillespie [1977] showed that the dynamics of a chemical reaction system are described by a continuous time Markov process with the transition rates given by the propensities. Thus, the probability of $P(\mathbf{n}, t)$ to find the system in state $\mathbf{n}$ at time $t$ obeys the chemical master equation.

$$
\frac{d P(\mathbf{n})}{d t}=\sum_{\alpha=1}^{R}\left[\lambda_{\alpha}\left(\mathbf{n}-\boldsymbol{\Delta}_{\mathbf{a}}\right) P\left(\mathbf{n}-\boldsymbol{\Delta}_{\mathbf{a}}\right)-\lambda_{\alpha}(\mathbf{n}) P(\mathbf{n})\right] .
$$

The master equation may be understood as a balance equation for the probability of finding the system in some state $\mathbf{n}$. The first term of the right hand side of equation (2.4) describes the gain in probability for systems to be in state $\mathbf{n}$ due to chemical reactions leading from other states $\mathbf{n}-\boldsymbol{\Delta}_{\mathbf{a}}$ into it. Accordingly the second term accounts for the loss in probability.

The chemical master equation contains a complete description of the stochastic reaction dynamics, including chemical noise which is due to the inherent stochasticity of single molecular reaction events. The discreteness of particle numbers is an essential ingredient of this type of noise. The strength of chemical noise can be quantitatively estimated in simple cases. As an example, consider the reaction scheme

$$
\begin{aligned}
& k_{+}: \quad \emptyset \rightarrow A \\
& k_{-}: \quad A \rightarrow \emptyset .
\end{aligned}
$$

The chemical Master equation corresponding to this reaction scheme is given by

$$
\begin{aligned}
\frac{d P(N, t)}{d t} & =J(N-1 \rightarrow N)-J(N \rightarrow N+1) \\
& =k_{+} P(N-1, t)+k_{-}(N+1) P(N+1, t)-\left(k_{+}+k_{-} N\right) P(N, t) .
\end{aligned}
$$

where $N$ denotes the number of $\mathrm{A}$ molecules and

$$
\begin{gathered}
J(N-1 \rightarrow N)=k_{+} P(N-1)-k_{-} N P(N) \\
J(N \rightarrow N+1)=k_{+} P(N)-k_{-}(N+1) P(N+1)
\end{gathered}
$$

are the net probability currents from $N-1$ to $N$ and from $N$ to $N+1$, respectively. To study the chemical noise in this simple case let us solve the Master equation under stationary conditions, i.e. for $d P / d t=0$. As no particles emerge from zero particle 
number or get lost at infinite particle number $J(N \rightarrow N+1)=J=0$ for all $N=$ $0,1,2, \ldots$ under stationary conditions. Thus

$$
k_{+} P_{\text {stat }}(N-1)-k_{-} N P_{\text {stat }}(N)=J=0
$$

or, equivalently

$$
P_{\text {stat }}(N)=\frac{k_{+}}{N k_{-}} P_{\text {stat }}(N-1)
$$

Iterating this equation we get

$$
P_{\text {stat }}(N)=\frac{1}{N !}\left(\frac{k_{+}}{k_{-}}\right)^{N} P_{\text {stat }}(0)
$$

and from the normalisation of probability $\sum_{N} P_{\text {stat }}(N)=1$ we get $P_{\text {stat }}(0)=\exp \left(-k_{+} / k_{-}\right)$. Thus, the particle numbers are distributed according to a Poisson law under stationary conditions. This implies

$$
\begin{gathered}
\langle N\rangle_{\text {stat }}=\frac{k_{+}}{k_{-}} \\
\sigma_{N}^{2}=\left\langle(N-\langle N\rangle)^{2}\right\rangle_{\text {stat }}=\frac{k_{+}}{k_{-}}
\end{gathered}
$$

If we follow several trajectories $N_{\alpha}(t), \quad \alpha=1, \ldots, M$ all starting from identical initial conditions, we get a typical spread in particle numbers around the expected mean value $\bar{N}=k_{+} / k_{-}$due to chemical noise, which can be characterised by

$$
\sqrt{\sigma_{N}^{2}} /\langle N\rangle=1 / \sqrt{\langle N\rangle}
$$

For $\bar{N}=5,10,20,50,200$ the corresponding typical spreads are $44.7 \%, 31.6 \%, 22.4 \%$, $14.1 \%$ and $7.1 \%$, respectively. Note, however, that the typical spread may not be the most conclusive measure of noise strength for the purposes of chemical signalling. Alternatively, consider the probability to get no particle at all (and therefore no further signalling!), $P(N=0)=\exp (-\bar{N})$. For $\bar{N}=5$ this takes on the value $\exp (-5)=0.0067$, so we have $\mathrm{a} \approx 0.7 \%$ chance to get no particle at all if we expect 5 particles to be present on average. For $\bar{N}=3,2,1$ this probability rises to $5 \%, 13.5 \%, 36.8 \%$, respectively. The triggering of many signal cascades depends on very low particle numbers, especially in the upstream regions of the cascade and in such cases, chemical noise can cause large fluctuations in the ultimate output controlled by the signalling cascade. 


\subsection{Operator Descriptions of the Chemical Master Equation: Single Compartment}

Let us now consider a general biochemical reaction system in a single well-stirred compartment with a dynamics described by the chemical master equation

$$
\frac{d P(\mathbf{n})}{d t}=\sum_{\alpha=1}^{R}\left[\lambda_{\alpha}\left(\mathbf{n}-\boldsymbol{\Delta}_{\mathbf{a}}\right) P\left(\mathbf{n}-\boldsymbol{\Delta}_{\mathbf{a}}\right)-\lambda_{\alpha}(\mathbf{n}) P(\mathbf{n})\right] .
$$

The rhs of the equation may be considered as the action of a linear operator on a discrete state space vector with components $P(\boldsymbol{n})$. Note that operators corresponding to chemical Master equations are composed from a few elementary operations:

a) the multiplication with $n_{i}^{\prime} \mathrm{s}$ or rather functions of the $n_{i}$ (the propensities)

b) shifts in particle numbers $\boldsymbol{n} \rightarrow \boldsymbol{n}+\boldsymbol{\Delta}_{\alpha}$.

If we want to exploit this point of view it is very convenient to introduce notations adapted to linear spaces. ${ }^{1}$ Therefore let us introduce a Dirac-style bra-ket notation, so that (abstract) state vectors are denoted by $|P\rangle$ and linear forms on the state space by $\langle\phi|$. In particular $\langle\boldsymbol{n} \mid P\rangle=P(\boldsymbol{n})$ are the components of $|P\rangle$ with respect to a base consisting of states, which represent non-fluctuating particle numbers. The corresponding state vectors $|\boldsymbol{n}\rangle$ form a base of mutually orthogonal vectors in the state space. We will make repeated use of the corresponding completeness relation $\sum_{\boldsymbol{n}}|\boldsymbol{n}\rangle\langle\boldsymbol{n}|=\mathbb{1}$. The basic operations a) and b) can be represented by operators

$$
\hat{n}_{i}\left|n_{1}, \cdots, n_{i}, \cdots n_{M}\right\rangle=n_{i}\left|n_{1}, \cdots, n_{i}, \cdots n_{M}\right\rangle
$$

and

$$
\hat{T}(\boldsymbol{\Delta})|\boldsymbol{n}\rangle=|\boldsymbol{n}+\boldsymbol{\Delta}\rangle
$$

and the Master equation may now be written in an abstract operator notation

$$
\partial_{t}|P\rangle=-\hat{H}|P\rangle=\sum_{\alpha=1}^{R}\left[\hat{T}\left(\boldsymbol{\Delta}_{\boldsymbol{\alpha}}\right)-1\right] \lambda_{\alpha}(\hat{\boldsymbol{n}})|P\rangle
$$

Note that $\left\langle\boldsymbol{n}\left|T\left(\boldsymbol{\Delta}_{\alpha}\right)\right| P\right\rangle=\left\langle\boldsymbol{n}-\boldsymbol{\Delta}_{\alpha} \mid P\right\rangle$ as long as all $n_{i}-\Delta_{\alpha, i}$ stay positive.

Further notice, that the shift operators may be written as products of elementary shift operators, which just change the particle numbers of a single species by \pm 1 :

$$
\begin{gathered}
\hat{T}_{i}^{+}\left|n_{1}, \cdots, n_{i}, \cdots n_{M}\right\rangle=\left|n_{1}, \cdots, n_{i}+1, \cdots n_{M}\right\rangle \\
\hat{T}_{i}^{-}\left|n_{1}, \cdots, n_{i}, \cdots n_{M}\right\rangle=\left|n_{1}, \cdots, n_{i}-1, \cdots n_{M}\right\rangle \text { for } n>0 .
\end{gathered}
$$

${ }^{1}$ The description presented here is a variant of the description put forward by [Doi, 1976] and [Peliti, 1984]. See [Cardy and Täuber, 1998] for a comprehensive review. 
so that $\hat{T}\left(\boldsymbol{\Delta}^{+}-\boldsymbol{\Delta}^{-}\right)=\prod_{i}\left(\hat{T}_{i}^{+}\right)^{\Delta_{i}^{+}}\left(\hat{T}_{i}^{-}\right)^{\Delta_{i}^{-}}$

To make further progress let us consider the generating function

$$
G(\boldsymbol{\varphi}, t):=\sum_{\boldsymbol{n}}\left(\prod_{i} \varphi_{i}^{n_{i}}\right) P(\boldsymbol{n}, t) .
$$

It can be used to obtain the expectation values of observables like moments $\left\langle n_{i}^{k}\right\rangle=$ $\sum_{\boldsymbol{n}} n_{i}^{k} P(\boldsymbol{n}, t)$ and correlation functions $\left\langle n_{i}^{k} n_{j}^{l} \cdots\right\rangle$ by differential operations at $\boldsymbol{\varphi}=1$ as follows,

$$
\left\langle n_{i}^{k} n_{j}^{l} \cdots\right\rangle=\left.\left(\varphi_{i} \frac{\partial}{\partial \varphi_{i}}\right)^{k}\left(\varphi_{j} \frac{\partial}{\partial \varphi_{j}}\right)^{l} \cdots G(\boldsymbol{\varphi}, t)\right|_{\boldsymbol{\varphi}=1} .
$$

Note that repeated differentiation at $\varphi=1$ generates

$$
\left\langle n_{i}\left(n_{i}-1\right)\left(n_{i}-2\right) \cdots\left(n_{i}-k\right)\right\rangle=\left.\left(\frac{\partial}{\partial \varphi_{i}}\right)^{k} G(\boldsymbol{\varphi}, t)\right|_{\boldsymbol{\varphi}=1} .
$$

Probabilities $P(\boldsymbol{n}, t)$ can also be directly obtained from $G(\boldsymbol{\varphi}, t)$ by differentiations with respect to the $\varphi_{i}$ 's at $\varphi=0$ :

$$
P(\boldsymbol{n}, t)=\left.\frac{1}{n_{1} ! n_{2} ! \cdots n_{M} !} \frac{\partial^{n_{1}}}{\partial \varphi_{1}^{n_{1}}} \cdots \frac{\partial^{n_{M}}}{\partial \varphi_{M}^{n_{M}}} G(\boldsymbol{\varphi}, t)\right|_{\boldsymbol{\varphi}=0}
$$

Note that the conservation of probability implies that

$$
G(\varphi=1, t)=1
$$

In the abstract bra-ket notation, the generating function corresponds to

$$
G(\varphi, t)=\langle\varphi \mid P\rangle
$$

where the state $|\varphi\rangle$ has components

$$
\langle\boldsymbol{n} \mid \boldsymbol{\varphi}\rangle=\prod_{i} \varphi_{i}^{n_{i}}
$$

To set up the evolution equation

$$
\partial_{t}\langle\boldsymbol{\varphi} \mid P\rangle=-\langle\boldsymbol{\varphi}|\hat{H}| P\rangle
$$

consider the following matrix elements of elementary shift operators $\hat{T}_{i}^{ \pm}$

$$
\begin{aligned}
\left\langle\varphi\left|\hat{T}_{i}^{+}\right| P\right\rangle & =\varphi_{i}\langle\varphi \mid P\rangle \\
\left\langle\varphi\left|\hat{T}_{i}^{-} \hat{n}_{i}\right| P\right\rangle & =\frac{\partial}{\partial \varphi_{i}}\langle\varphi \mid P\rangle
\end{aligned}
$$


whereas

$$
\left\langle\boldsymbol{\varphi}\left|\hat{n}_{i}\right| P\right\rangle=\varphi_{i} \frac{\partial}{\partial \varphi_{i}}\langle\varphi \mid P\rangle
$$

These relations are easily obtained by inserting the completeness relation $\sum_{n}|\boldsymbol{n}\rangle\langle\boldsymbol{n}|=\mathbb{1}$. Note that it is $\hat{T}_{i}^{-} \hat{n}_{i}$ that corresponds to a simple partial derivative. Thus it requires a bit of disentangling (or operator ordering) to get the evolution equation of the characteristic function as a partial differential equation, because the Master equation is naturally represented in terms of shift and particle number operators. Luckily, the problem is simple in the most important special case. Note that

$$
\left(\hat{T}_{i}^{-} \hat{n}_{i}\right)^{k}\left|\cdots n_{i} \cdots\right\rangle=n_{i}\left(n_{i}-1\right)\left(n_{i}-2\right) \cdots\left(n_{i}-k+1\right)\left|\cdots n_{i}-k \cdots\right\rangle
$$

Thus, if a reaction $R_{\alpha}$ involves destruction of $\mathrm{k}$ particles of species $i$ and if the propensity has the form $\lambda_{\alpha}(\boldsymbol{n})=n_{i}\left(n_{i}-1\right)\left(n_{i}-2\right) \cdots\left(n_{i}-k+1\right) \tilde{\lambda}_{\alpha}(\boldsymbol{n})$ (as it should on the basis of kinetic collision theory of reactions), then the corresponding part of the Master equation Hamiltonian is given by

$$
\left[\left(\hat{T}_{i}^{-}\right)^{k}-1\right] \lambda_{\alpha}(\hat{\boldsymbol{n}})=\left[1-\left(\hat{T}_{i}^{+}\right)^{k}\right]\left(\hat{T}_{i}^{-} \hat{n}_{i}\right)^{k} \tilde{\lambda}_{\alpha}(\hat{\boldsymbol{n}})
$$

which leads to a term

$$
\left[1-\varphi_{i}^{k}\right]\left(\frac{\partial}{\partial \varphi_{i}}\right)^{k} \tilde{\lambda}_{\alpha}\left(\varphi \partial_{\varphi}\right) G(\varphi)
$$

in the evolution equation of $G$. Here $\varphi \partial_{\varphi}$ is short hand notation for the set of all $\varphi_{i} \partial_{\varphi_{i}}$.

Restricting our considerations to this most important case, the Hamiltonian $\hat{H}$ takes on the form:

$$
\hat{H}=-\sum_{\alpha=1}^{R}\left[\prod_{i}\left(\hat{T}_{i}^{+}\right)^{\Delta_{\alpha . i}^{+}} \prod_{j}\left(\hat{T}_{j}^{-}\right)^{\Delta_{\alpha, j}^{-}-1}\right]\left[\prod_{k} n_{k}\left(n_{k}-1\right) \cdots\left(n_{k}-\Delta_{\alpha, k}^{+}\right)\right] \tilde{\lambda}_{\alpha}(\boldsymbol{n})
$$

which may be rewritten into the form

$$
\hat{H}=-\sum_{\alpha=1}^{R}\left[\prod_{i}\left(\hat{T}_{i}^{+}\right)^{\Delta_{\alpha, i}^{+}}-\prod_{j}\left(\hat{T}_{j}^{+}\right)^{\Delta_{\alpha, j}^{-}}\right]\left[\prod_{k}\left(\hat{T}_{k}^{-} \hat{n}_{k}\right)^{\Delta_{\alpha, k}^{-}}\right] \tilde{\lambda}_{\alpha}(\boldsymbol{n})
$$

From this form we may read off the evolution equation for $G=\langle\varphi \mid P\rangle$ :

$$
\partial_{t} G(\boldsymbol{\varphi})=\sum_{\alpha=1}^{R}\left[\prod_{i}\left(\varphi_{i}\right)^{\Delta_{\alpha . i}^{+}}-\prod_{i}\left(\varphi_{i}\right)^{\Delta_{\alpha . i}^{-}}\right]\left[\prod_{j}\left(\partial_{\varphi_{j}}\right)^{\Delta_{\alpha . j}^{-}}\right] \tilde{\lambda}_{\alpha}\left(\boldsymbol{\varphi} \partial_{\boldsymbol{\varphi}}\right) G(\boldsymbol{\varphi})
$$

and the corresponding Hamiltonian in $\varphi$-representation $H\left(\boldsymbol{\varphi}, \boldsymbol{\partial}_{\boldsymbol{\varphi}}\right)$. The conservation of probability (stated as $\partial_{t}\langle\boldsymbol{\varphi}=1 \mid P\rangle=-\langle\boldsymbol{\varphi}=1|\hat{H}| P\rangle=0$ requires that every admissible Hamiltonian has to vanish if we set all the $\varphi_{i}=1$

$$
H\left(\varphi=1, \partial_{\varphi}\right)=0 \text {. }
$$

Let us set up a few examples, which may serve as templates to construct the Hamiltonian for complex situations: 
Monomolecular decay

$$
\begin{aligned}
& k_{-}: \quad A \rightarrow \emptyset \\
& \hat{H} \hat{=}-k_{-}\left(1-\varphi_{A}\right) \partial_{\varphi_{A}}
\end{aligned}
$$

Spontaneous creation

$$
\begin{gathered}
k_{+}: \quad \emptyset \rightarrow A \\
\hat{H} \hat{=}-k_{+}\left(\varphi_{A}-1\right)
\end{gathered}
$$

Spontaneous creation of pairs

$$
\begin{gathered}
k_{+}: \quad \emptyset \rightarrow A+A \\
\hat{H} \hat{=}-k_{+}\left(\varphi_{A}^{2}-1\right)
\end{gathered}
$$

Dimer decay

$$
\begin{gathered}
k_{-}: \quad A+A \rightarrow \emptyset \\
\hat{H} \hat{=}-k_{-}\left(1-\varphi_{A}^{2}\right) \partial_{\varphi_{A}}^{2}
\end{gathered}
$$

Bimolecular decay

$$
\begin{gathered}
k_{-}: \quad A+B \rightarrow \emptyset \\
\hat{H} \stackrel{=}{-} k_{-}\left(1-\varphi_{A} \varphi_{B}\right) \partial_{\varphi_{A}} \partial_{\varphi_{B}}
\end{gathered}
$$

Monomolecular reaction

$$
\begin{gathered}
k: \quad A \rightarrow B \\
\hat{H}=\hat{=}-k\left(\varphi_{B}-\varphi_{A}\right) \partial_{\varphi_{A}}
\end{gathered}
$$

Bimolecular reaction

$$
\begin{gathered}
k: \quad A+B \rightarrow C \\
\hat{H} \bumpeq-k\left(\varphi_{C}-\varphi_{A} \varphi_{B}\right) \partial \varphi_{A} \partial \varphi_{B}
\end{gathered}
$$

Finally, as an example of a reaction system, we consider a standard enzymatic reaction. 


\section{Enzymatic reaction scheme}

$$
\begin{array}{ll}
k_{+}: & A+E \rightarrow A E \\
k_{-}: & A E \rightarrow A+E \\
k_{p}: & A E \rightarrow E+P
\end{array}
$$

The first reaction corresponds to

$$
\hat{H}_{1}=-k_{+}\left(\varphi_{A E}-\varphi_{A} \varphi_{E}\right) \partial_{\varphi_{A}} \partial_{\varphi_{E}}
$$

The second reaction implies the decay of $A E$ together with the creation of $A$ and $E$ :

$$
\hat{H}_{2}=-k_{-}\left(\varphi_{A} \varphi_{E}-\varphi_{A E}\right) \partial_{\varphi_{A E}}
$$

and the third reaction couples the vanishing of $A E$ to the creation of $E$ and $P$

$$
\hat{H}_{3}=-k_{p}\left(\varphi_{P} \varphi_{E}-\varphi_{A E}\right) \partial_{\varphi_{A E}}
$$

The complete Hamiltonian is the sum of the three parts $\hat{H}=\hat{H}_{1}+\hat{H}_{2}+\hat{H}_{3}$.

\subsubsection{Functional Integral Representation}

In this section we want to replace the operator description by an equivalent representation of $G(\phi, t)$ in terms of multiple, in fact infinitely many, integrals. This representation is a very convenient starting point for systematic approximations in a large variety of systems [Kleinert, 1993]. To free our notation from unnecessary complications we first consider a single species. The general case will be achieved by obvious addition of indices in the end result. We want to find a representation of

$$
G(\varphi, t)=\left\langle\varphi\left|e^{-\hat{H} t}\right| P_{0}\right\rangle
$$

where $\left|P_{0}\right\rangle$ is the initial probability state vector. For definiteness, we will choose either fixed particle numbers, i.e. $\left|P_{0}\right\rangle=\left|n_{0}\right\rangle$ or a Poisson distribution $P_{0}(n)=\left\langle n \mid P_{0}\right\rangle=$ $\exp (-\bar{n}) \bar{n}^{n} / n$ !. Note that the corresponding generating function is given by $G_{0}(\varphi)=$ $\exp (-\bar{n}) \sum_{n=0}^{\infty}(\varphi \bar{n})^{n} / n !=\exp [\bar{n}(\varphi-1)] .^{2}$

Let us introduce operators $\hat{\varphi}$ and $\hat{\eta}=-i \partial / \partial_{\varphi} .{ }^{3}$ The additional imaginary unit in the definition of $\hat{\eta}$ will make intermediary steps more comprehensive. Later on, it is possible to return to a definition without the factor of $i$. We will make extensive use of completeness relations corresponding to the eigenstates of $\hat{\varphi}$ and $\hat{\eta}$. Eigenstates of $\hat{\varphi}$ are

\footnotetext{
${ }^{2}$ For those acquainted with quantum mechanics it may be helpful to consider the evolution equation for $G(\varphi)$ as a Schrödinger equation in imaginary time and take over the well-known procedure to construct Feynman's path integral representation of quantum mechanics.

${ }^{3}$ In quantum mechanics they would correspond to the position and momentum operator, respectively.
} 
defined by $\hat{\varphi}|\varphi\rangle=\varphi|\varphi\rangle$, where $\varphi$ can be any real number. They obey the orthogonality and normalisation relations

$$
\left\langle\varphi_{1} \mid \varphi_{2}\right\rangle=\delta\left(\varphi_{1}-\varphi_{2}\right)
$$

The $|\varphi\rangle$ form a complete set of base vectors, the corresponding completeness relation in Dirac-style bra-ket notation takes on the form

$$
\mathbb{1}=\int|\varphi\rangle d \varphi\langle\varphi|
$$

It is easy to see now that the $\hat{\eta}$ eigenstates $|\eta\rangle$ correspond to plane waves in $|\varphi\rangle$ representation ${ }^{4}$.

$$
\langle\varphi \mid \eta\rangle=\frac{1}{\sqrt{2 \pi}} e^{i \varphi \eta}
$$

The $|\eta\rangle$ also form a complete, orthonormal set and the corresponding completeness relation is

$$
\mathbb{1}=\int|\eta\rangle d \eta\langle\eta|
$$

Before we start let us point out that the construction it will require the operator $\hat{H}$ to be ordered in such a way that all $\hat{\varphi}$ are found left from all $\hat{\eta}$ operators $^{5}$. So, should $\hat{H}$ not be given in this form, it first has to be reordered.

We start by writing the time evolution operator in Eq. (2.6) as a "time-sliced product"

$$
=\left\langle\varphi\left|\prod_{k=0}^{N} e^{-\hat{\mathbf{H}} \delta t}\right| G_{0}\right\rangle
$$

with a small time step $\delta t=t /(N+1)$. Let us now repeatedly introduce the completeness relation Eq.(2.7) in between the factors of the time sliced product

$$
=\int\left(\prod_{k=0}^{N} d \varphi_{k}\right)\left\langle\varphi \mid \varphi_{N}\right\rangle\left\langle\varphi_{N}\left|e^{-\hat{H} \delta t}\right| \varphi_{N-1}\right\rangle\left\langle\varphi_{N-1}\right| \cdots\left\langle\varphi_{1}\left|e^{-\hat{H} \delta t}\right| \varphi_{0}\right\rangle\left\langle\varphi_{0} \mid P_{0}\right\rangle
$$

As all $\hat{\varphi}$-operators are left from $\hat{\eta}$ - operators we have

$$
\left\langle\varphi_{k}\right| e^{-H(\hat{\varphi}, \hat{\eta}) \delta t}=\left\langle\varphi_{k}\right| e^{-H\left(\varphi_{k}, \hat{\eta}\right) \delta t}+O\left(\delta t^{2}\right)
$$

To get rid of the $\hat{\eta}$ operators, we again insert completeness relations this time corresponding to the $|\eta\rangle$ basis so that

$$
\left\langle\varphi_{k} \mid e^{-H\left(\varphi_{k}, \eta\right) \delta t} \eta_{k-1}\right\rangle=\left\langle\varphi_{k} \mid \eta_{k-1}\right\rangle e^{-H\left(\varphi_{k}, \eta_{k-1}\right) \delta t}
$$

\footnotetext{
${ }^{4}$ At this point we need the factor of $i$ in the definition of $\eta$. A definition without this imaginary factor would lead to so called coherent states as eigenstates. A derivation of the functional integral representation can also be given (see e.g. [Popov, 1981]) using these states, but it requires somewhat more insight into quantum mechanics

${ }^{5}$ Other operator ordering schemes are also possible (and lead to equivalent results), but the Hamiltonian always has to follow a definite operator ordering scheme
} 
Thus we get a product of factors of the form

$$
\left\langle\varphi \mid \eta_{N}\right\rangle\left\langle\eta_{N} \mid \varphi_{N}\right\rangle\left\langle\varphi_{N}\left|e^{-\delta t \hat{H}}\right| \eta_{N-1}\right\rangle \cdots\left\langle\eta_{1} \mid \varphi_{1}\right\rangle\left\langle\varphi_{1}\left|e^{-\delta t \hat{H}}\right| \eta_{0}\right\rangle\left\langle\eta_{0} \mid \varphi_{0}\right\rangle
$$

Apart from the ends, the repeated motif of this product is

$$
\left\langle\eta_{k} \mid \varphi_{k}\right\rangle\left\langle\varphi_{k}\left|e^{-\delta t \hat{H}}\right| \eta_{k-1}\right\rangle=\frac{1}{2 \pi} e^{-i \varphi_{k}\left(\eta_{k}-\eta_{k-1}\right)} e^{-\delta t H\left(\varphi_{k}, \eta_{k-1}\right)}
$$

Collecting all the factors we get an approximate representation of $G$ in terms of multiple integrals, which will become exact in the limit of infinitesimal time step, i.e. $N \rightarrow \infty$

$$
G(\varphi, t)=\lim _{N \rightarrow \infty} \int \prod_{k=0}^{N} \frac{d \varphi_{k} d \eta_{k}}{2 \pi} \exp (-S) G_{0}\left(\varphi_{0}\right)
$$

with

$$
S=\sum_{k=1}^{N} i \varphi_{k}\left(\eta_{k}-\eta_{k-1}\right)+H\left(\varphi_{k}, \eta_{k-1}\right) \delta t+i \eta_{0} \varphi_{0}-i \eta_{M} \varphi-\bar{n}\left(\varphi_{0}-1\right)
$$

for a Poissonian initial state. Finally we may change back to the operator $\partial_{\varphi}$ of our stochastic problem by putting $\eta \rightarrow-i \eta^{6}$. In the continuum limit, the stochastic action $S$ may then be written in the form:

$$
S=\int_{0}^{t_{f}} d t[\varphi(t) \dot{\eta(t)}+H(\varphi, \eta)]+\varphi(0)(\eta(0)-\bar{n})-\eta\left(t_{f}\right) \varphi
$$

Note that we may also write the action in the equivalent form

$$
S=\int_{0}^{t_{f}} d t[-\eta(t) \dot{\varphi(t)}+H(\varphi, \eta)]+\eta\left(t_{f}\right)\left(\varphi\left(t_{f}\right)-\varphi\right)-\varphi(0) \bar{n}
$$

which corresponds to a partial integration of the first term of the action.

As promised, the generalisation to more species is straightforward:

$$
S=\int_{0}^{t_{f}} d t\left[\boldsymbol{\varphi}(t) \cdot \dot{\boldsymbol{\eta}}+H(\boldsymbol{\varphi}, \boldsymbol{\eta})+\boldsymbol{\varphi}(0) \cdot(\boldsymbol{\eta}(0)-\overline{\boldsymbol{n}})-\boldsymbol{\eta}\left(t_{f}\right) \cdot \boldsymbol{\varphi}\right.
$$

\subsubsection{Rate equations and noise induced effects}

A first important application of the functional integral representation is the precise definition of rate equations emerging from a stochastic formulation and a systematic small noise

\footnotetext{
${ }^{6}$ The convergence of the functional integral has to be assured by proper rotations in the complex planes of $\varphi_{k}$ and $\eta_{k}$ variables anyhow.
} 
expansion. If fluctuations become small (compared to the typical values of $S$ ), the functional integral is dominated by contributions near the saddle point trajectory $\left(\boldsymbol{\varphi}_{c}(t), \boldsymbol{\eta}_{c}(t)\right)$ of $S$. The saddle point approximation of the functional integral is well-studied and corresponds to the semiclassical approximation in quantum mechanics. The equations of motion determining the saddle point trajectories are obtained from $\delta S /\left.\delta \varphi_{i}(t)\right|_{c}=0$ and $\delta S /\left.\delta \eta(t)\right|_{c}=0$ :

$$
\begin{aligned}
\dot{\varphi}_{c, i} & =\frac{\partial H}{\partial \eta_{c, i}} \\
\dot{\eta}_{c, i} & =-\frac{\partial H}{\partial \varphi_{c, i}}
\end{aligned}
$$

These equations have to be supplemented by boundary conditions (see equation (2.12) and equation (2.13))

$$
\begin{gathered}
\eta_{c, i}(0)=\bar{n}_{i} \\
\varphi_{c, i}\left(t_{f}\right)=\varphi_{i},
\end{gathered}
$$

where $\varphi_{i}$ are the variables of the generating function $G\left(\boldsymbol{\varphi}, t_{f}\right)$, which we want to calculate.

Note that the system of equations of motion may be considered as a system of Hamilton equations of a classical mechanical system with a Hamiltonian $H$. Below we will make use of this analogy, identifying coordinates with $\varphi_{c, i}$ and momenta with $-\eta_{c, i}$. Note furthermore, that the quantity $E=H\left(\boldsymbol{\varphi}_{c}(t), \boldsymbol{\eta}_{c}(t)\right.$ ("energy" in classical mechanics) is a constant of motion.

Given $S_{c}=S\left[\boldsymbol{\varphi}_{\boldsymbol{c}}, \boldsymbol{\eta}_{c}\right]$, the saddle point approximation implies that

$$
G(\boldsymbol{\varphi}, t) \approx C \exp \left[-S_{c}(\boldsymbol{\varphi})\right]
$$

7 Thus the average particle numbers are approximated by

$$
\left\langle n_{i}\right\rangle=\left.\varphi_{i} \frac{\partial G(\boldsymbol{\varphi}, t)}{\partial \varphi_{i}}\right|_{\varphi=1} \approx-\left.\varphi_{i} \frac{\partial S_{c}(\boldsymbol{\varphi})}{\partial \varphi_{i}}\right|_{\varphi=1}=\eta_{c, i} .
$$

The latter equality holds because it is a well-known result of classical mechanics that on the classical trajectory the derivative of the classical action with respect to coordinates $\left(\varphi_{i}\right)$ gives the momenta $\left(-\eta_{i}\right)$ (see textbooks on classical mechanics, for example [Landau and Lifschitz, 1997]). Given this relation, we want to find a closed equation of motion for $\left\langle n_{i}\right\rangle_{c}=\eta_{c, i}$ from equation (2.14). Note that due to the structure of the Hamiltonian, in particular of the terms $\left[\varphi_{k}^{\Delta^{+}}-\varphi_{i}^{\Delta^{-}}\right] \eta_{i}^{\Delta^{-}}$, the derivative $\partial H / \partial \eta_{i}$ will always vanish at $\varphi_{i}=1$. Thus there is always a classical solution

$$
\boldsymbol{\varphi}_{c}(t)=1 \quad \text { tor all } \quad 0 \leq t \leq t_{f}
$$

\footnotetext{
${ }^{7}$ The constant $C=\exp (-S(1))$ has to guarantee the conservation of probability.
} 
and the remaining equation

$$
\dot{\eta}_{i}=-\left.\frac{\partial H}{\partial \varphi_{i}}\right|_{\varphi=1}
$$

is the rate equation, emerging from the stochastic description in the limit of small noise.

An important thing to note here is that the rate equation is determined by the Hamiltonian in its normally ordered form. In these cases, the Hamiltonian has to be reordered, before the rate equations can be read off and this may lead to surprising, counterintuitive additional terms.

\subsubsection{Noise induced effects}

Signals, which are detected by receptors of the biological cell always carry a certain amount of (extrinsic) noise. A signal may consist of chemical as well as physical stimuli (like light, heat, or electro-chemical signals further downstream the signalling cascade), which influence the propensities of reactions in the signalling cascade. Here, we want to demonstrate, that the functional integral description of chemical reactions is very useful in detecting noise induced effects, which sometimes appear to be counter-intuitive, because they improve the cellular response instead of blurring or disrupting it. Such noise-induced effects are well-known in systems described by Langevin and Fokker-Planck equations (see for instance [Garcia-Ojalvo et al., 1992] or [Santos and Sancho, 1999]) ${ }^{8}$, but are only rarely studied in the context of more complicated chemical reactions. To be specific, let us consider the following simple reaction system

$$
\begin{gathered}
k_{1}: A \rightarrow 2 A \\
k_{2}: A+A \rightarrow \emptyset \\
k_{3}: A \rightarrow \emptyset
\end{gathered}
$$

The Hamiltonian of this system is given by

$$
H_{0}=-k_{1}\left(\varphi^{2}-\varphi\right) \eta-k_{2}\left(1-\varphi^{2}\right) \eta-k_{3}(1-\varphi) \eta
$$

and the rate equation is

$$
\dot{\eta}=-\left.\frac{\partial H}{\partial \varphi}\right|_{\varphi=1}=\left(-k_{3}+k_{1}\right) \eta-2 k_{2} \eta^{2}
$$

The stationary states of this equation are given by

$$
\eta_{1}=0
$$

\footnotetext{
${ }^{8}$ In a biological context the constructive nature of noise is best known from the study of stochastic resonance, which accounts for the phenomenon that correctly tuned fluctuations lead to the enhancement of an external signal [Gammaitoni et al., 1998].
} 
and

$$
\eta_{2}=\frac{k_{1}-k_{3}}{k_{2}} \text { for } k_{1}>k_{3}
$$

The latter state may considered as the active response of the system to signals. Every one of the three propensities could in principle be modified by some signal, depending on the receptor mechanism. Let us first consider the case, where $k_{3}=k+\xi(t)$ and $\xi(t)$ is Gaussian noise with a very short correlation time $\tau_{\xi}$ as a typical simple model of environmental noise ${ }^{9}$. In the limit $\tau_{\xi} \rightarrow 0$ we get the idealised model of Gaussian white noise, completely characterised by the average

$$
<\xi(t) \xi\left(t^{\prime}\right)>_{\xi}=C_{0} \delta\left(t-t^{\prime}\right)
$$

Averages of observables over internal and external noise may be obtained from the averaged generating function $\left\langle G(\varphi)>_{\xi}\right.$. Let us therefore consider the $\xi$-average within the functional integral representation. There is a subtle point here, which becomes more obvious when considering the averaging of the time sliced product. In our case it takes on the form

$$
\prod_{k=1}^{N} \exp \left[-\hat{H}_{0} \delta t-\Delta w_{k}(1-\hat{\varphi}) \hat{\eta}\right]
$$

with

$$
\Delta w_{k}=\int_{t_{k-1}}^{t_{k}} \xi(t) d t
$$

$\hat{H}_{0}$ denotes the noise free part of the Hamiltonian. The $\Delta w_{k}$ are independently distributed Gaussian stochastic variables with zero average $\left\langle\Delta w_{k}\right\rangle=0$ and variance

$$
<\left(\Delta w_{k}\right)^{2}>_{\xi}=C_{0} \delta t,
$$

in accordance with Eq.(2.17), so that each factor of the time sliced product can be averaged separately. After inserting the completeness relations, we evaluate the factors

$$
<\left\langle\varphi_{k}\left|\exp \left[-\hat{H}_{0} \delta t-\Delta w_{k}(1-\hat{\varphi}) \hat{\eta}\right]\right| \eta_{k-1}\right\rangle>_{\xi}
$$

by expanding the exponential neglecting terms of $O\left(\delta t^{2}\right)$. Note, however, that this expansion now requires to keep terms of $O\left(\Delta w_{k}^{2}\right)$, which after averaging become terms of $O(\delta t)$. Thus we get

$$
\exp \left[-\hat{H}_{0} \delta t+\Delta w_{k}(1-\hat{\varphi}) \hat{\eta}\right]=-\left[1+\hat{H}_{0} \delta t-\frac{C_{0}}{2}(1-\hat{\varphi}) \hat{\eta}(1-\hat{\varphi}) \hat{\eta} \delta t\right]+O\left(\delta t^{2}\right)
$$

The result of the averaging may be considered as an addition to the Hamiltonian $\hat{H}_{0}$. Note, however, that the additional term is not ordered in such a way that all the $\hat{\varphi}$

\footnotetext{
${ }^{9}$ Note that an increase in signal corresponds to a decrease in the degradation rate $k_{3}$.
} 
operators are on the left to all the $\hat{\eta}$ operators. Thus we have to reorder this term before we can actually evaluate the expression Eq.(2.19). The correctly ordered Hamiltonian looks as follows:

$$
\hat{H}=\hat{H}_{0}+\frac{C_{0}}{2}(1-\hat{\varphi}) \hat{\eta}-\frac{C_{0}}{2}(1-\hat{\varphi})^{2} \hat{\eta}^{2} .
$$

The second term on the r.h.s. of this equation corresponds to a renormalisation (shift) of the net rate of $A$-production and it will show up in the rate equation by the replacement

$$
\left(-k_{3}+k_{1}\right) \rightarrow\left(-k_{3}+k_{1}+\frac{C_{0}}{2}\right)
$$

The $3^{r} d$ term on the r.h.s. will not show up in the rate equation and may be considered as a modification of the internal chemical noise. It is somewhat counter-intuitive that the effect of the noise is to enhance the signal (i.e. lead to a decrease of the degradation rate).

Such noise induced effects have been widely studied using Langevin equations. When using this formalism, it is well-known that the renormalisation of rates appears and leads to qualitative changes in the system dynamics for spatially extended systems. ${ }^{10}$ Examples include noise-induced phase transitions, noise-induced front propagation and noise induced generation of patterns. A survey of such phenomena is given in [Sancho and Garcia-Ojalvo, 2000].

The added value of the present approach is not only that it makes it easy to transcribe many of the results obtained for Langevin equations to chemical reaction-diffusion systems described by Master equations. It also gives direct and easy access to noise induced renormalisation due to more complex couplings between the signal and the receptor. The general recipe to establish additional terms due to noisy external signals should be clear by now. If we can split the Hamiltonian into a noise-free part and a coupling of extrinsic (Gaussian white) noise to the system as follows

$$
\hat{H}=\hat{H}_{0}+\xi(t) \hat{H}_{1}
$$

and then repeat the above steps we end up with a Hamiltonian

$$
\tilde{H}=\hat{H}_{0}-\frac{C_{0}}{2} \hat{H}_{1}^{2}=\hat{H}_{0}+\Delta \hat{H}_{\text {noise }}
$$

after averaging over $\xi$. Finally we have to reorder $\hat{H}_{1}^{2}$ appropriately, bringing all $\eta$ operators to the right.

Let us illustrate the procedure for the other two propensities of our reaction system. If external noise couples to the autocatalytic reaction $k_{1} \rightarrow k_{1}+\xi(t)$ we get

$$
\Delta \hat{H}_{\text {noise }}=-\frac{C_{0}}{2} \hat{\varphi}(\hat{\varphi}-1) \hat{\eta} \hat{\varphi}(\hat{\varphi}-1) \hat{\eta}
$$

\footnotetext{
${ }^{10}$ For single compartment systems, the presence of noise prevents an actual phase transition and the modified noise counteracts the renormalisation effect for long times.
} 
which after reordering takes on the form

$$
\Delta \hat{H}_{\text {noise }}=-\frac{C_{0}}{2}\left[\hat{\varphi}(\hat{\varphi}-1)(2 \hat{\varphi}-1) \hat{\eta}+\hat{\varphi}^{2}(1-\hat{\varphi})^{2} \hat{\eta}^{2}\right]
$$

Replacing the operators by numbers and differentiating with respect to $\varphi$ at $\varphi=1$ we get the additional terms in the rate equation. Here

$$
\left.\Delta \dot{\eta}\right|_{\text {noise }}=\frac{C_{0}}{2} \eta
$$

i.e. the same renormalisation as induced by $k_{3}$ noise. (Note, however, that the additionally induced terms look completely different in these two cases, indicating a different renormalisation of intrinsic noise)

Finally, if the extrinsic noise couples by $k_{2} \rightarrow k_{2}+\xi(t)$ we have

$$
\Delta \hat{H}_{\text {noise }}=-\frac{C_{0}}{2}\left(1-\hat{\varphi}^{2}\right) \hat{\eta}\left(1-\hat{\varphi}^{2}\right) \hat{\eta} .
$$

Reordering this term we get

$$
\Delta \hat{H}_{\text {noise }}=-\frac{C_{0}}{2}\left[\left(1-\hat{\varphi}^{2}\right)^{2} \hat{\eta}^{4}-4\left(1-\hat{\varphi}^{2}\right) \hat{\varphi} \hat{\eta}^{3}-2\left(1-\hat{\varphi}^{2}\right) \hat{\eta}^{2}\right]
$$

and this leads to

$$
\left.\Delta \dot{\eta}\right|_{\text {noise }}=\frac{C_{0}}{2}\left[8 \eta^{3}+4 \eta^{2}\right]
$$

Note that these terms change the global dynamical behaviour of the system qualitatively. The $\eta^{3}$-term leads to an inevitable instability and a run-away behaviour, whereas the $\eta^{2}$ term shifts the binary decay towards global instability. Thus the system is structurally unstable against noise in the binary decay term. This is also a somewhat surprising result, which shows that certain receptor mechanisms require further elements of the biochemical network to stabilise it against extrinsic noise.

\subsection{Spatially Inhomogeneous Reaction Systems}

The above derivation of the chemical master equation heavily relies on the assumption that the propensity functions only depend on the current state of the system. In particular this means that the spatial distribution of molecules within the reaction medium equilibrates on a much faster timescale than the timescale on which chemical reactions occur. However, since the stirring of the medium depends on the diffusive transport of the particles, this condition is not necessarily met when studying chemical signalling. For cytosolic proteins the diffusion coefficients typically lie in a range between 10 and $50 \mu^{2} \mathrm{~s}^{-1}$. Since the average distance covered by diffusion is proportional to the square root of time. i.e.

$$
\left\langle L^{2}\right\rangle \propto D t
$$


it takes a protein approximately 2 to $10 \mathrm{~s}$ to cover a distance of $10 \mu \mathrm{m}$, which is a realistic length scale for eukaryotic cells. Hence, a simulation method, which totally neglects space would only be justified, if the time in between any two reaction would be on the same order of time. Clearly this is not the case for biological system in which typical rate constants vary in a range from 0.1 to $100 \mathrm{~s}^{-1}$.

That the cellular environment does not constitute a homogeneous reaction medium becomes even more obvious, if one also bears in mind, that diffusion is highly restricted in biological cells due to molecular crowding and constraints imposed by geometry [Elowitz et al., 1999]. It is therefore obvious that the underlying geometry needs to be accounted for when modelling chemical signalling. Within the mesoscopic approach presented here this is accomplished by subdividing the overall reaction volume $\Omega$ into $\mathrm{N}$ non-overlapping, coupled, well-stirred reaction volumes $\Omega\left(\mathbf{r}_{\mathbf{k}}\right) k=1, \ldots, N$. The subvolumes are labelled by a representative point $\mathbf{r}_{\mathbf{k}}$ from their interior. In particular we assume for the time being that the virtual subvolumes are cubic in shape and that they are all of the same size. This restriction will be abandoned in Chapter 5, when we will cover the spatial modelling of chemical signalling cascades in greater detail. In order for our approach to produce physically meaningful results, it is now essential that the molecules are homogeneously distributed in each subvolume. Above, we have argued that this is the case if the typical time for a particle to cross a subvolume by three-dimensional diffusion is much smaller than the time in between two successive reactions. For cubic subvolumes of volume $\Omega\left(\mathbf{r}_{\mathbf{k}}\right)$ and side length $\Omega\left(\mathbf{r}_{\mathbf{k}}\right)^{1 / 3}$ we therefore have to require that

$$
\Omega\left(\mathbf{r}_{\mathbf{k}}\right)^{2 / 3} / D \ll \lambda^{-1} .
$$

Let us now extend the above formalism to this new situation, where we have a collection of well-stirred reaction subvolumes $\Omega\left(\boldsymbol{r}_{k}\right), k=1,2, \cdots$, which make up the total reaction volume $\Omega$. The state space of the system is spanned by the ket vectors

$$
\left|\boldsymbol{n}\left(\boldsymbol{r}_{1}\right), \boldsymbol{n}\left(\boldsymbol{r}_{2}\right), \cdots\right\rangle \text {. }
$$

Chemical reactions are considered as strictly local within each subvolume, i.e. the Master equation would take on the form

$$
\partial_{t}|P\rangle=-\hat{H}^{\text {react }}|P\rangle=-\sum_{k} \hat{H}_{k}^{\text {react }}|P\rangle
$$

(where $\hat{H}_{k}^{\text {react }}$ acts only on particle numbers in subvolume $k$ ) were it not for the thermal motion of molecules, which leads to incoming and outgoing particles in each subvolume via its boundaries. In the mesoscopic description we model this motion as a random hopping between adjacent subvolumes. The hopping probabilities have to be determined from the requirement that the molecular motion on scales large compared to subvolume 
sizes has to be diffusive. As noted above, we will in this chapter only consider the simple situation of cubic subvolumes of equal size, which fill up the reaction volume (a cube) with a regular cubic lattice. The representative points $\boldsymbol{r}_{k}$ are placed at the centre of the cubic subvolumes so that the $\boldsymbol{r}_{k}$ make up a simple cubic point lattice with lattice sites at $a\left(m_{1} \boldsymbol{e}_{1}+m_{2} \boldsymbol{e}_{2}+m_{3} \boldsymbol{e}_{3}\right)$ with integer number $m_{1}, m_{2}, m_{3}$. The hopping process may be described by a collection of first order chemical reactions. There is one reaction for every nearest neighbour to a lattice site. The neighbours of site $\boldsymbol{r}_{k}$ are located at $\boldsymbol{r}_{k}+a \boldsymbol{e}_{\mu}, \mu=1,2 \cdots, 6$, where the $\boldsymbol{e}_{\mu}$ correspond to $\pm \boldsymbol{e}_{1}, \pm \boldsymbol{e}_{2}, \pm \boldsymbol{e}_{3}$. The reactions $R_{i, k, \mu}$ are characterised by

$$
w_{i, k, \mu}: \quad A_{i}\left(\boldsymbol{r}_{k}\right) \rightarrow A_{i}\left(\boldsymbol{r}_{k}+\boldsymbol{e}_{\mu}\right)
$$

with propensities $w_{i, k, \mu}=K_{i, k, \mu} n_{i}\left(\boldsymbol{r}_{k}\right)$ which reflect the first order kinetics resulting from the independent molecular motions of individual molecules. The Hamiltonian corresponding to these monomolecular reactions takes on the form

$$
\hat{H}^{h o p}=-\sum_{i=1}^{M} \sum_{k} \sum_{\mu=1}^{6} K_{i, k, \mu}\left[\hat{\varphi}_{i}\left(\boldsymbol{r}_{k}+\boldsymbol{e}_{\mu}\right)-\hat{\varphi}_{i}\left(\boldsymbol{r}_{k}\right)\right] \hat{\eta}_{i}\left(\boldsymbol{r}_{k}\right)
$$

or equivalently

$$
\hat{H}^{h o p}=-\sum_{i=1}^{M} \sum_{k} \sum_{\mu=1}^{6} K_{i, k, \mu}\left[\hat{\eta}_{i}\left(\boldsymbol{r}_{k}+\boldsymbol{e}_{\mu}\right)-\hat{\eta}_{i}\left(\boldsymbol{r}_{k}\right)\right] \hat{\varphi}_{i}\left(\boldsymbol{r}_{k}\right)
$$

For long wavelength fluctuations in a homogeneous medium (for which $K_{i, k, \mu}=K_{i}$ ) we may pass to a (formal) continuum limit by the replacements

1.) from sum over $\boldsymbol{r}_{k}$ to integral $\sum_{\boldsymbol{r}_{k}} \rightarrow a^{3} \int d^{3} \boldsymbol{r}$

2.) from particle numbers to densities $\eta_{i}\left(\boldsymbol{r}_{k}\right) \rightarrow a^{3} \eta(\boldsymbol{r})$

3.) $\boldsymbol{\varphi}\left(\boldsymbol{r}_{k}\right) \rightarrow \boldsymbol{\varphi}(\boldsymbol{r})$.

The sum over lattice directions is a discretized version of the Laplace operator on the cubic lattice and we replace

$$
\frac{1}{a^{2}} \sum_{\mu}\left[\hat{\eta}_{i}\left(\boldsymbol{r}_{k}+\boldsymbol{e}_{\mu}\right)-\hat{\eta}_{i}\left(\boldsymbol{r}_{k}\right)\right] \rightarrow \Delta \hat{\eta}(\boldsymbol{r})+O\left(a^{2}\right)
$$

The reaction part of the Hamiltonian becomes an integral of a Hamiltonian density $\hat{h}^{\text {react}}$, so that the continuum limit of $\hat{H}$ takes on the form

$$
\hat{H}=-\sum_{i=1}^{M} \frac{K_{i}}{a^{2}} \int d^{3} \boldsymbol{r} \varphi(\boldsymbol{r}) \nabla^{2} \eta(\boldsymbol{r})+\int d^{3} \boldsymbol{r} \hat{h}^{r e a c t}(\boldsymbol{\varphi}(\boldsymbol{r}), \boldsymbol{\eta}(\boldsymbol{r}))
$$

To ensure diffusive motion on the long length-scales we identify

$$
\frac{K_{i}}{a^{2}} \rightarrow D_{i} \text { Diffusion constant of species i }
$$


in the continuum limit. The semiclassical evolution equations corresponding to this Hamiltonian takes on the form of a general system of reaction diffusion equations

$$
\partial_{t} \eta_{i}(\boldsymbol{r}, t)=D_{i} \nabla^{2} \eta_{i}(\boldsymbol{r}, t)-\left.\frac{\partial h^{\text {react }}}{\partial \varphi_{i}(\boldsymbol{r}, t)}\right|_{\boldsymbol{\varphi}=1}
$$

\subsection{Monte Carlo Algorithms}

In the following we will present three different algorithms for generating trajectories of stochastic processes whose dynamics are described by a master equation. In the present case the underlying master equation will of course be the reaction diffusion master equation from above. The algorithms to be presented are called the Direct Method (DM), the Next Subvolume Method (NSM), and the Logarithmic Classes Method (LCM). With the help of an ensemble of trajectories one is able to estimate the statistics of the observables. In the ideal case of the model capturing the essence of the underlying system, the generated trajectories correspond to observations that could in principle be made in real biological experiments.

All algorithms which we will use for simulating chemical signalling work by first determining the time at which the next event occurs. Subsequently they decide what kind of an event this will be, i.e. it is decided whether a reaction occurs or whether a particle is moved from one subvolume to another. The algorithms therefore belong to the general class of variable time step methods. Determining the time at which the next event will happen can be easily done because the times in between successive events are exponentially distributed. To see this, let us first consider the general case of a Markov process in which the probability of observing a transition from one state to another within the time interval $d \tau$ is given by the probability $r d \tau$. Consequently the probability for observing a state change exactly after $n+1$ time steps of length $d \tau$ is given by

$$
p=(1-r d \tau)^{n} r d \tau \text {. }
$$

If we now set $\tau=(n+1) d \tau$ and take the limit $d \tau \rightarrow 0$, while leaving $\tau$ fixed, it immediately follows that $n \rightarrow \infty$. This however means that the probability of a transition exactly after a time interval of length $\tau$ is given by

$$
p(\tau) d \tau=r \exp \{-r \tau\} d \tau .
$$

Hence, if we want to calculate the residence time of a system to be in some state, we need to draw an exponentially distributed random number with parameter $r$. In the present case of modelling reaction diffusion systems, the parameter $r$ will either refer to the reactivity of a subvolume $\gamma_{k}$, defined by the sum over all diffusion and reaction rates, i.e.

$$
\gamma_{k}=\sum_{i=1}^{M} \sum_{\mu=1}^{6} w_{i, k, \mu}+\sum_{\alpha} \lambda_{\alpha}^{k}
$$


or $r$ will refer to the overall reactivity of the system $\Gamma$, which in return is given by the sum over all the subvolumes' reactivities, i.e.

$$
\Gamma=\sum_{k} \gamma_{k}
$$

Notice, that the average waiting times in between two events in a subvolume $k$ or in the entire system are given by the inverse of $\gamma_{k}$ and $\Gamma$ respectively. This is simply due to the fact that the first moment of an exponential distribution with parameter $r$ is given by $1 / r$.

\subsubsection{Direct Method}

The Direct Method (DM) was originally proposed by D. Gillespie in 1976 for the simulation of well stirred, single compartment chemical reaction systems [Gillespie, 1976]. It can however easily be extended to systems with several well stirred subvolumes. As already indicated above the DM consists of the repetition of two steps. First it calculates how long the system will stay in the current state before a new state is selected in the second step. For calculating the time at which the next event will take place, it draws a random number $\tau$ from an exponential distribution with the global weight $\Gamma$ as a parameter. Subsequently it sets the system time $t$ to $t=t+\tau$. In the second step the algorithms then chooses the subvolume in which the event takes place. In particular the algorithm has to choose a subvolume $i$ such that the probability of selecting that subvolume is given by the ratio of its weight to the overall reactivity of the system. This task can easily be accomplished with the linear selection scheme. The general idea behind this method is given in Figure 2.1, in which the reactivities of each subvolume are visualised as subintervals on a line, which has a total length equal to the total reactivity $\Gamma$. In a implementation of the

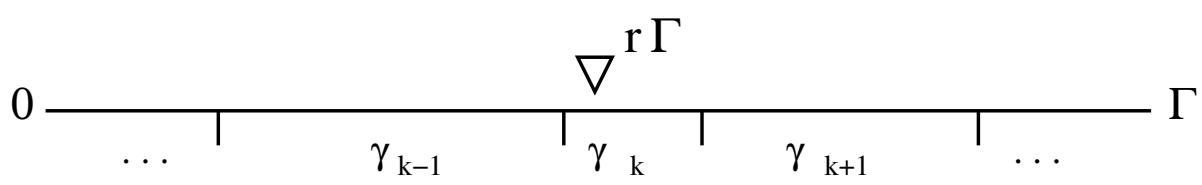

Figure 2.1: Selecting a subvolume with a probability proportional to is contribution to the overall weight is accomplished by multiplying the overall reactivity $\Gamma$ with a uniformly distributed random number $r$. Obviously the probability that the point $r \Gamma$ lies in the boundaries of intervals $\gamma_{k}$ is given by $\gamma_{k} / \Gamma$.

linear selection method one begins with multiplying the overall reactivity of the system $\Gamma$ with a random number $r$ which is uniformly drawn from the unit interval. This product is assigned to some variable $s=\Gamma r$. Subsequently the reactivities of the subvolumes $\gamma_{k}$ are subtracted one after another from $s$ until its value drops below 0 for the first time. The subvolume $k$ for which this is the case marks the reaction cell in which the next event will 
take place. From the graphical representation of the linear selection method displayed in Figure 2.1 it should also be clear that one can subtract the reactivities of the subvolumes in any order from $s$ because $r$ is a uniformly distributed random number. Choosing an event from that subvolume has again to be done in such a way that the probability of choosing a particular event is given by its contribution to the overall reactivity of the subvolume. Hence, the linear selection scheme is employed anew. Again, this involves the drawing of a uniformly distributed random number $r_{2}$ from the interval $[0,1]$, which this time has to be multiplied with the weight of the chosen subvolume $\gamma_{k}$, such that one has $w=r_{2} \gamma_{k}$. The individual propensities of the potential reaction and diffusion events are then subtracted until $w$ drops below 0 . The event $j$ for which this is the case marks the chosen event. After executing it, the weights of the affected subvolumes are updated, i.e. one has to recalculate the reactivity of one subvolume if a reaction event occurred and two reactivities have to be recalculated in case that a diffusion event occurred. Finally after having updated the global weight as well, the overall procedure is repeated until the stopping criteria is met. A recipe of the algorithm is given in the box on the next page. 


\section{Recipe Direct Method}

1. Initialisation: Calculate propensities of all reaction and diffusion events and calculate the weights of all subvolumes. Calculate global weight $\Gamma$.

2. Determine $\tau$ according to an exponential distribution with parameter $\Gamma$ and set global time $t$ to $t=t+\tau$.

3. Choose the subvolume in which the next event will take place with the help of a linear selection method:

- Draw a normalised uniformly distributed random number $r$ and set $X=$ $r * \Gamma$ and set $k=-1$.

- while $(\mathrm{X}>0)$

$* k=k+1$

* $X=X-\gamma_{k}$

4. Choose which event will take place within subvolume $i$ with the help of a linear selection method:

- Draw a normalised uniformly distributed random number $r_{2}$ and set $Y=$ $r_{2} * \gamma_{k}$ and set $j=-1$.

- while $(\mathrm{Y}>0)$

$* j=j+1$

$* Y=Y-x_{j}$ (with $x_{j}$ denoting either the propensity of a diffusion or of a reaction event)

5. Execute event $j$ and update affected weights; repeat from 2 if stopping criteria are not met.

\subsubsection{Next Subvolume Method}

The Next Subvolume Method (NSM) was developed by J. Elf and M. Ehrenberg in 2004 [Elf and Ehrenberg, 2004]. It uses a slightly different approach than the DM, resembling that of the First Reaction Method (FRM), which was also proposed by D. Gillespie in 1976 for the simulation of well stirred reaction systems [Gillespie, 1976]. This latter algorithm was formerly considered as being less efficient than the DM and was therefore never really used. Instead of using the global reactivity for calculating the next event time as in the Direct Method, the FRM generates putative next event times for each possible reaction. Subsequently it chooses the reaction channel for which the next event 
time is minimal. The NSM is similar to the FRM in that it also calculates putative next event times. However, it does this for every subvolume and not for every event. For each subvolume $k$ the putative next event time $t_{k}$ is given by $t_{k}=t+\tau$ with $t$ being the global time of the system. $\tau$ is a random number drawn from an exponential distribution with the subvolume's reactivity $\gamma_{k}$ as parameter, i.e. $p_{k}(\tau)=\gamma_{k} \exp \left(-\gamma_{k} \tau\right)$. After having all the putative event times at hand, the problem consists of finding the subvolume for which the next event time is minimal. In order to efficiently select this subvolume, the NSM administers the event times of all the subvolumes in a binary heap, which is a data structure commonly used to store items in a prescribed order. In particular a binary heap is a binary tree with an additional constraint imposed on the way in which the values are stored in the data structure. This is known as the heap condition. In the present case this condition consists of the requirement that the values stored within the children of a node $i$ are always larger than the value stored in the node itself. That way the subvolume in which the next event will take place is easily identified as it is always stored in the root of the heap. An example of a binary heap as it is used in this algorithm is given in Figure 2.2, in which one assumes that the reaction medium is made up of four subvolumes $a, b, c$, and $d$ in which the next events occur at times $1.3,1.7,0.2$, and 1.1. The idea of using a binary heap in kinetic Monte Carlo simulations is not at all new.

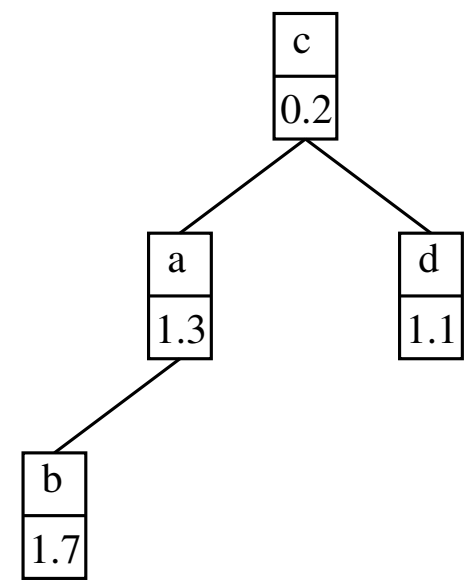

Figure 2.2: An example of a binary heap as it is used in our simulation environment. Note that a node consists of two parts. Apart from the event time it also stores an identifier referring to the subvolume for which it stores this value.

In the context of simulating chemical signalling it has first been employed in the Next Reaction Method, which was developed by Gibson and Bruck in 2000 for the simulation of single compartment systems. After having accessed the subvolume, whose next event time is stored in the root of the heap, the algorithm first sets the global time to $t=t_{k}$. Then the algorithm needs to decide which event will occur in the selected subvolume. Again this has to be done in such a way that the probability of selecting an event is 
equal to the event's contribution to the overall weight of the subvolume. Consequently, this task is accomplished by employing the linear selection method. Hence, this part of the algorithm is similar to the fourth step in the Direct Method. Subsequently after the execution of an event at most two putative event times have to be updated; one if the event was a reaction and two in the case that a diffusion event occurred. Furthermore, before the algorithm is repeated, one has to update the heap at most at two distinct nodes, for which standard algorithms exist [Sedgwick and Van Wyk, 2002]. A detailed outline of the algorithm is again given as a recipe and can be found below.

\section{Recipe Next Subvolume Method}

1. Initialisation: Calculate propensities of all reaction and diffusion events and calculate weights of all subvolumes.

2. Calculate the first event time for each subvolume by drawing a random number according to an exponential distribution with parameter $\gamma_{k}$. Store subvolumes in such a way in a binary heap so that the ancestors of an arbitrary node always have a later event time than their predecessor. This way the subvolume for which the next event occurs first is stored within the top node.

3. Subvolume $k$ stored within the top element of the heap marks the subvolume in which the next event occurs. Set global time t to $t=\tau_{k}$.

4. Determine next event within subvolume $k$ according to a linear selection method described within the Direct Method.

5. Determine new next event times for the affected subvolumes. If necessary update ordering of the nodes within the heap so that all ancestors have again a later event times than their predecessors.

6. Repeat from 3 if stopping criteria are not met.

\subsubsection{Logarithmic Classes Method}

An approach almost forgotten today is the algorithm first proposed by [Fricke and Wendt, 1995] and which has been described by Schnakenberg in 1995 [Schnakenberg, 1995]. In the following we will refer to this algorithm as the Logarithmic Classes Method (LCM). At the bottom of this algorithm is the insight that the linear selection scheme used in the Direct Method has to sum on average over $N / 2$ reactivities in order to select the subvolume in which the next event will occur. In order to avoid this computational bottleneck, the LCM is based on the idea of using some sort of presorting when selecting a subvolume. This is achieved by binning the subvolumes according to their reactivities 

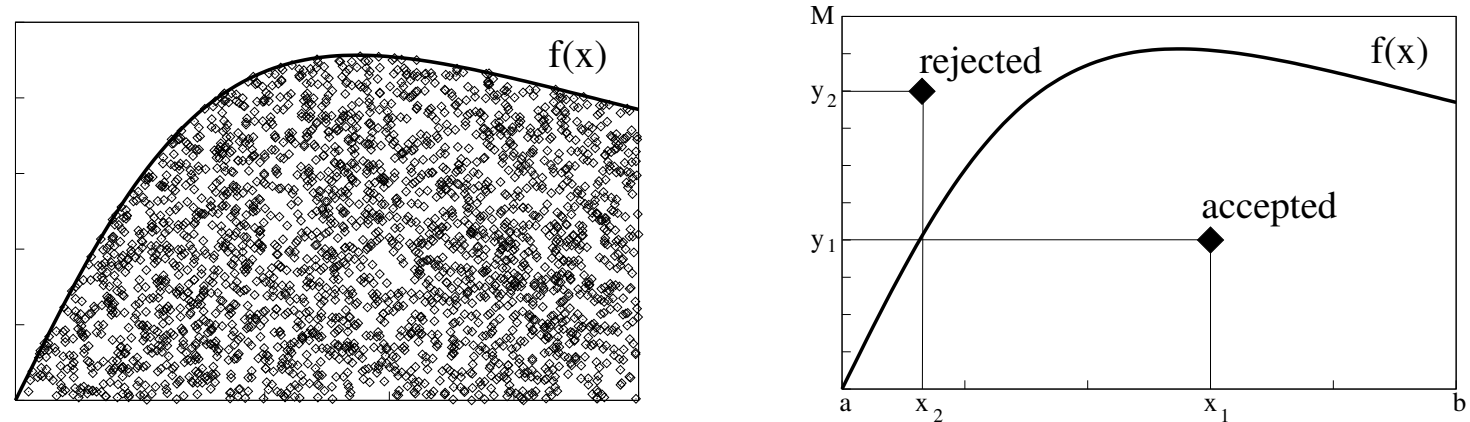

Figure 2.3: Left part: A set of points $\left(x_{i}, y_{i}\right)$ evenly fills the area underneath some function $f(x)$, if the $x_{i}$ are random numbers distributed according to $f(x)$ and if the $y_{i}$ are generated in such a way that they are evenly distributed in the interval $\left[0, f\left(x_{i}\right)\right]$. Right part: Visualisation of the von Neumann method: $x_{1}$ is accepted as a realisation of a random number generated according to $f(x)$ because $y_{1}<f\left(x_{1}\right) . x_{2}$ is rejected because $y_{2}>f\left(x_{2}\right)$.

into logarithmic classes. In particular, a subvolume $k$ belongs to the logarithmic class $C_{y}$, if the following relation for its reactivity $\gamma_{k}$ holds true:

$$
2^{y} \leq \gamma_{k}<2^{y+1}
$$

Hence, $C_{y}$ defines a set of subvolumes in which the reactivities differ at most by a factor of 2. Moreover the algorithm keeps track of the overall reactivity of a logarithmic class $\Gamma_{y}=\sum_{k \in C_{y}} \gamma_{k}$ as well as of the system's total reactivity $\Gamma=\sum_{C_{y}} \Gamma_{y}$. The last quantity is again used for calculating the time at which the next event occurs. This is done as described under the second point in the recipe of the Direct Method. Choosing the subvolume is now split into two steps. First a logarithmic class has to be selected before one actually selects the subvolume in which the next event will occur. The first step is accomplished by using a linear selection procedure such that a logarithmic class $y$ is chosen according to its contribution to the overall reactivity $\Gamma_{y} / \Gamma$. Subsequently a subvolume $k$ has to be drawn out of the obtained logarithmic class according to its contribution to the logarithmic class's overall reactivity $\left(\gamma_{k} / \Gamma_{y}\right)$. For this task the algorithm employs the von Neumann rejection method. This method dates back to 1947 and allows for the generation of random numbers distributed according to any given probability density. It is based on the observation that if we are given a large set of random numbers $A$ which are generated according to the probability density $p(x)$, then the area underneath $p(x)$ will be evenly filled with points $(x, y)$, if we draw for every realisation $x \in A$ a second random number $y=p(x) r$ with $r$ being a uniformly distributed random number between 0 and 1. We have visualised this finding in the left part of Figure 2.3. Hence, if we are given some function $f(x)$ defined on the interval $[a, b]$ for which an upper bound $M$ is known to exists, we can generate random numbers according to this function in the following 
way: First a random number $r_{1}$ is generated, which is uniformly distributed in the interval $[a, b]$. However, this random number will only be accepted as a correct random number, if the relation $r_{2} \leq f\left(r_{1}\right)$ holds true for a second random number uniformly drawn from the interval $[0, M]$. Otherwise, $r_{1}$ is rejected and the procedure has to be repeated. We have visualised this algorithm in the right part of Figure 2.3, in which $x_{1}$ is a random number that can be accepted, whereas $x_{2}$ has to be rejected. Applying the von Neumann algorithm to the present context of selecting a subvolume from a logarithmic class, this implies that one first randomly chooses a subvolume $k$ from the selected logarithmic class with probability $1 / n_{y}$ ( $n_{y}$ denoting the total number of subvolumes within logarithmic class $y$ ). Subsequently one compares the subvolume's reactivity with the product of a second uniformly distributed random number $r_{2}$ and the upper bound of the subvolumes' reactivities. According to the definition of a logarithmic class, this last quantity is given by $2^{y+1}$. Hence, if $r_{2} 2^{y+1} \leq \gamma_{k}$ an event is linearly selected and executed in subvolume $k$. Otherwise the entire method has to be repeated. After executing the event and updating the weights, the algorithm has to check, whether the affected subvolume(s) still belong to the correct logarithmic class(es). If necessary, the updated subvolumes have to be reassigned to different logarithmic classes, before the algorithm can be repeated. As for the previous two algorithms this method can be found in the form of a recipe below.

\section{Recipe Logarithmic Classes Method}

1. Initialisation: Calculate propensities of all reaction and diffusion events and calculate weights of all subvolumes. Calculate global weight and set up logarithmic classes table.

2. Determine $\tau$ and set global time $\mathrm{t}$ to $t=t+\tau$

3. Select a logarithmic class $l$ according to a linear selection scheme.

4. Select a subvolume from the logarithmic class $l$ with the help of a von Neumann rejection method:

- Select randomly a subvolume $k$ from the logarithmic class $l$.

- Draw a normalised uniformly distributed random number $r$. If $r 2^{l+1} \leq \gamma_{k}$, subvolume $k$ is selected. Else repeat from step 4 .

5. Select an event to be executed within subvolume $k$ by a linear selection method.

6. Update affected subvolumes and weights. Repeat from 2 if stopping criteria is not met. 


\section{Variation 1: Comparing the efficiency of the different approaches}

in which we compare the computational efficiency of the three algorithms presented in the previous chapter. The Logarithmic Classes Method turns out to be the most efficient method for simulating chemical signalling cascades. We conclude this chapter by presenting an optimisation of this algorithm.

3.1 Introduction .................. 39

3.2 Comparison .................. 40

3.2 .1 Autocatalytic waves . . . . . . . . . . . . . 40

3.2 .2 Localised spots . . . . . . . . . . . . . . . . . . . . . . 41

3.3 Discussion ................... . 43

3.3.1 Analysis of the different Monte Carlo methods . . . . . 43

3.3.2 Improved version of the Logarithmic Classes Method . . 45

\subsection{Introduction}

In the last chapter we have presented three algorithms with the help of which one is capable of simulating chemical signalling in a spatially resolved manner. That these algorithms are not equally well suited for this task will become evident in this chapter, in which we will compare the computational efficiency of the different methods. Nowadays, apart from detailed experimental input, computational efficiency has turned out to be the most important bottleneck for stochastic simulations of reaction diffusion systems in the field of systems biology [Lok, 2004].

For comparing the computational efficiency of the three algorithms, two different scenarios were used. In the first scenario the event probabilities are roughly homogeneously distributed across the reaction medium whereas the second scenario displays great inhomogeneities in the spatial distribution of the weights of the reactioncells. Thereby we cover the two extremes which can be encountered when dealing with spatially resolved chemical signalling systems. All three algorithms were implemented in the $\mathrm{C}++$ programming language and compiled using the GNU C compiler assembly version 4.0.1 
with optimisation flag -O2. Simulations were subsequently performed on an IBM T30 notebook with a $2.3 \mathrm{MHz}$ Intel Pentium III Mobile CPU with $768 \mathrm{MB}$ of RAM.

Before we start, notice that the reaction diffusion systems which we used for comparing the algorithms are all fairly simple. This is due to the fact that we want to compare the algorithms' performance on choosing the subvolume in which the next event will take place, because this is the most time consuming step in all the three stochastic methods. An increase in system size will foremost lead to an increase in the computing time used for selecting a subvolume. To ensure that only the selection of the subvolume is compared, all three methods use the same algorithm for choosing a diffusion or reaction event. As can be seen in chapter 2, a linear selection scheme was always used for this task. Using a more sophisticated method for selecting an event would therefore not change the results of this comparison but only increase the overall efficiency of all the three algorithms used for this study.

\subsection{Comparison}

\subsubsection{Autocatalytic waves}

As a first test suite the spreading of an autocatalytic wave on a two dimensional grid is considered. This reaction diffusion system involves two chemical species denoted as active $(\mathrm{A})$ and inactive $(\mathrm{I})$ which have the same diffusion coefficients $D_{A}=D_{I}=20$ (in dimensionless units). The number of molecules is changed by either one of the following two reactions

$$
\begin{array}{lll}
A+I & \stackrel{k}{\rightarrow} & A+A \\
A+I & \stackrel{r}{\rightarrow} & I+I,
\end{array}
$$

for which the reaction rates are equal to $k=0.02$ and $r=0.01$ (again in dimensionless units). A rectangular grid with corners $(0,0),\left(0, L_{y}\right),\left(L_{x}, 0\right)$, and $\left(L_{x}, L_{y}\right)$ in a rectangular $(x, y)$ coordinate system is used as a reaction volume. The system sizes are varied from $32 \times 16$ to $2048 \times 1024$ gridcells. In the beginning all subvolumes attached to the left boundary extending in the y-direction from $(0,0)$ to $\left(0, L_{y}\right)$ only contain $\mathrm{A}$ molecules and no I molecules. In the rest of the grid the opposite holds true. There the density of I molecules is set to 1 whereas the density of $A$ molecules is equal to 0 . Having prepared the system in this way, a travelling wave carried by $A$ particles will evolve propagating with constant velocity into the positive $x$ direction. Visualising this system after a certain amount of time, gives rise to a snapshot as shown in left part of Figure 3.2. In that figure we use grey scales to code for the concentration of active molecules. Those parts of the medium which contain a high concentration of active molecules are dark whereas the absence of active molecules is indicated in white.

In Figure 3.1 the time needed to perform $10^{5}$ Monte Carlo steps is plotted as a function of system size. The red curve stands for the time needed by the LCM and the blue curve 


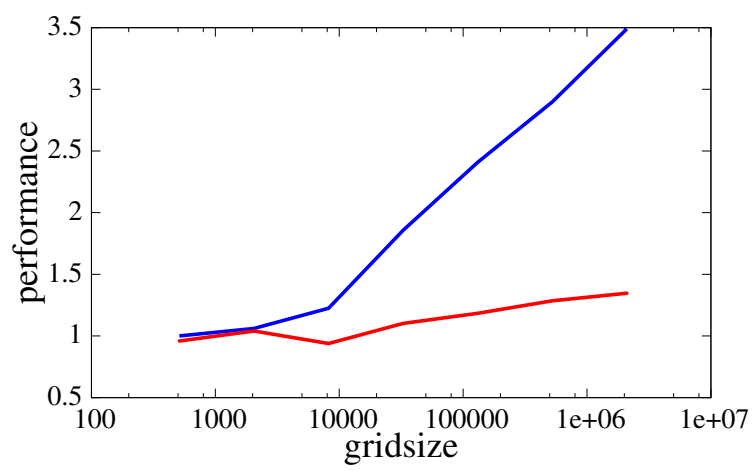

Figure 3.1: Time needed by the Next Subvolume and Logarithmic Classes Method to perform 100,000 Monte Carlo steps when simulating the reaction system stated in equation 3.1. The time is measured as a function of the number of reaction cells within the mesh

shows the performance of the NSM. The results are normalised such that unity is equal to the time needed by the Next Subvolume Method to perform $10^{5}$ Monte Carlo steps on the smallest grid. Note that we have not included the computing time needed by the Direct Method in Figure 3.1 because it drastically increases with increasing system sizes. For the smallest systems of a size of $32 \times 16$ reaction cells the Direct Method takes the same amount of computing time. However, for a system composed of $512 \times 256$ reaction cells it already takes more than 100 times as long as the other two methods! Comparing the efficiency of the Logarithmic Classes Method and the Next Subvolume Method reveals that the former always needs less computing time. For small system sizes (less then 4096 reaction cells) the difference between the two methods is only marginal. Starting with system sizes around $2^{13}$ subvolumes the Next Subvolume method becomes more and more inefficient. Finally, for the largest simulated system consisting of $2^{21}$ reaction cells, this method is more than two and a half times slower than the simulation using the Logarithmic Classes Method. Interestingly, in contrast to the Next Subvolume Method the computing time needed by the Logarithmic classes Method stays almost constant. Overall the computing time of this method only increases by roughly $40 \%$ when increasing the system from $2^{9}$ to $2^{21}$ subvolumes. As opposed to that the Next Subvolume Method consumes 250\% more computing time when comparing the largest with the smallest system.

\subsubsection{Localised spots}

Whereas the reactivities did not differ strongly across the medium in the previous example we will now consider a reaction diffusion system exhibiting large inhomogeneities. For this purpose we use the reaction diffusion system which was presented in [Bettelheim and Lehmann, 1999] and [Shnerb et al., 2003]. In this system particles of molecular species 

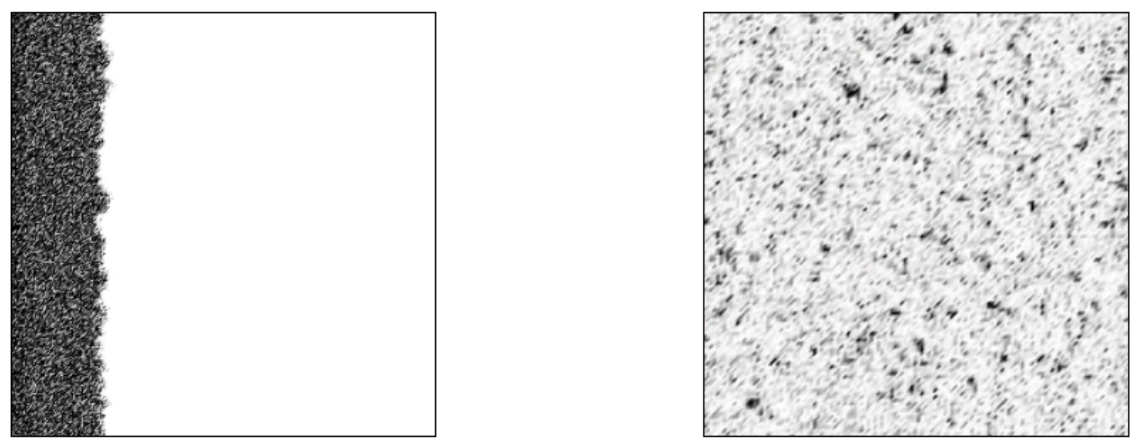

Figure 3.2: Snapshots of the two systems used for comparing the efficiency of the different algorithms. White regions indicate the absence of active molecules whereas an increasing darkness codes for an increasing presence of these molecules. The left plot visualises a catalytic wave travelling from the left to the right and the right part shows the pattern arising in the localised spots reaction diffusion system.

$A$ depend upon particles of species $F$ for reproduction. In particular the system is made up of the following two reactions:

$$
\begin{aligned}
A+F & \stackrel{k}{\rightarrow} A+A+F \\
A & \stackrel{r}{\rightarrow} \emptyset .
\end{aligned}
$$

The reaction and diffusion rates were chosen according to [Shnerb et al., 2003] who used the dimensionless values $k=13$ and $r=8$ for the reaction rates and $D_{A}=10$ and $D_{F}=0.2$ for the diffusion rates. With only a few $F$ particles being randomly distributed across the medium, this system gives rise to islands with high numbers of $A$ molecules surrounded by a desert in which there are virtually no molecules. Of course, islands can only evolve around $F$ molecules as they catalyse the reproduction of $A$. As in the previous case, the system was simulated on two dimensional grids of varying sizes ranging from $32 \times 16$ to $2048 \times 1024$ reaction cells. A snapshot of how the system looks like, when the performance is measured can be seen in the right part of Figure 3.2. Again a grey scale scheme is used for depicting the concentration of A molecules. Regions with a high A concentration are dark whereas regions containing no A molecules are light. Obviously there are only a few spots in the reaction system, at which the concentration of the active molecules is high. It is at these places where most of the reaction and diffusion events will take place. 


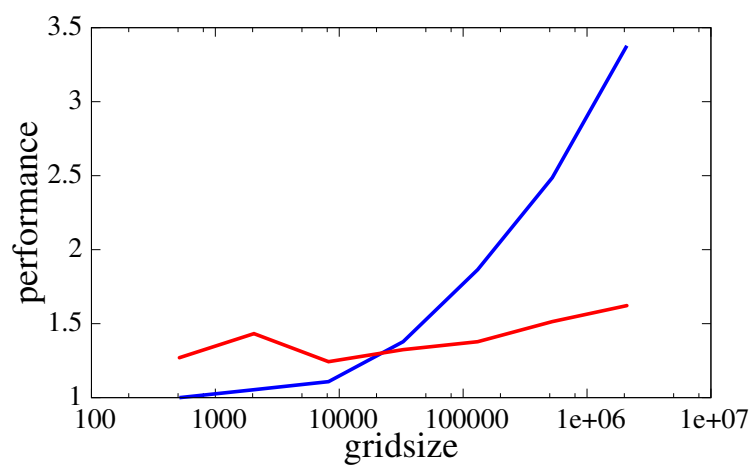

Figure 3.3: Time needed by the Next Subvolume and Logarithmic Classes Method to perform 100,000 Monte Carlo steps when simulating the reaction system stated in equation 3.2. The time is measured as a function of the number of reaction cells within the mesh.

The normalised results are displayed in Figure 3.3. Again unity refers to the time needed by the Next Subvolume method to perform $10^{5} \mathrm{MC}$ steps on the smallest grid and the blue curve stands for the NSM whereas the red curve accounts for the LCM. Notice that the time needed by the Direct Method for large systems is again beyond all evil so that we again only compare the Next Subvolume Method with the Logarithmic Classes Method. The results of this comparison for this system are somewhat different from the previous case. For small systems having less than $256 \times 128$ reaction cells the Next Subvolume Method is in fact faster than the Logarithmic Classes Method. For larger systems the latter method becomes again more efficient. However, the discrepancy between the two methods is not as strong as in the previous case. For the largest system simulated here the Logarithmic Classes Method is only twice as fast as the Next Subvolume Method. Furthermore, one can again observe that the logarithmic classes method does not depend as strongly as the Next Subvolume Method on the number of subvolumes in the system.

\subsection{Discussion}

\subsubsection{Analysis of the different Monte Carlo methods}

When comparing the different Monte Carlo methods for simulating reaction diffusion systems, we have seen that one should not use a naive adaptation of Gillespie's Direct Method to reaction diffusion systems. It is always a lot less efficient than the other two algorithms. Notice, that also the improved version of the Direct Method proposed by Cao et al. [2004] will not be applicable for simulating spatially extended signalling systems. This optimised version of the Direct Method was proposed for simulating spatially homogeneous reaction systems. It increases the efficiency of Gillespie's original algorithm by 
storing the reaction propensities in an ordered fashion such that the largest propensities are always subtracted first from the global weight. That way the chance of deciding in the first steps which event will take place, is greatly increased. This method may work fine when one has to maintain a limited number of reaction propensities in the correct order. However, maintaining the subvolumes ordered according to their reactivity is a computationally demanding task because this number easily becomes larger than $10^{5}$ when simulating signalling cascades in a realistic setting.

Overall, when simulating chemical signalling by an exact stochastic method, the Next Subvolume Method or even better the Logarithmic Classes Method should be preferred. The comparison of these two methods in the previous section revealed that the latter method is always more efficient than the former, when large reaction systems are simulated.

Overall the observed behaviour can easily be understood, if one analyses to which complexity classes the different algorithms belong. The Direct Method is most time consuming because in each propagation step it has to subtract on average $n / 2$ terms ( $n$ being the number of reaction cells within the system) from the global reactivity in order to determine in which subvolume the next event will take place. Hence, the Direct Method belongs to the complexity class $O(n)$. As compared to this, it takes the Next Subvolume Method only $O\left(\log _{2} n\right)$ steps to update the binary heap in the worst case scenario. This is simply due to the fact that the binary heap used for storing the reactivities of the subvolumes contains $\log _{2} n$ layers. Hence, in the case that the reaction time in the subvolume referred to by the top node of the heap is changed after an event in such a way that it needs to be propagated down to the lowest layer of the heap, the algorithms will perform $\log _{2} n$ comparisons. It seems as if this algorithm approaches this behaviour for large systems as a straight line can be fitted to the data (see Figure 3.1). Finally, the performance of the Logarithmic Classes Method depends on the linear selection scheme used for choosing a logarithmic class as well as on the rejection level of the von Neumann algorithm. In the two systems presented above there were never more than twelve different logarithmic classes. Since the reactivities within the classes by definition never differ by more than a factor of two, both steps are highly efficient. Overall, the complexity of the LCM is given by $O(L)+O(r(L))$, where $L$ denotes the number of logarithmic classes and $r(L)$ accounts for the number of rejections within each von Neumann step. Unlike the other two methods this algorithm therefore does not depend directly on the number of subvolumes within the system, which makes it superior to its competitors. To summarise these results we restate the complexity classes of the three different algorithms in Table 3.1 .

In the introduction to this chapter we have already pointed out that the efficiency of the stochastic algorithms used for simulating chemical signalling mainly depends on the number of subvolumes included in the system. As opposed to that the number of chemical species and reactions is of minor importance. To elaborate on this point, consider for instance the recent work by [Fange and Elf, 2006] in which a chemical 


\begin{tabular}{lr}
\hline Algorithm & Complexity Class \\
\hline Direct Method & $O(n)$ \\
Next Subvolume Method & $O(\log n)$ \\
Logarithmic Classes Method & $O(L)+O(r(L))$ \\
\hline
\end{tabular}

Table 3.1: Complexity classes of the different algorithms. Whereas the first two method scale with the number of gridcells $n$ the complexity class of the Logarithmic Classes Method is proportional to the number of logarithmic classes $L$ and to the number of rejections occurring within the von Neumann algorithm $r(L)$.

signalling cascade (the Min system) in a bacterial cell (Escherichia coli) was simulated. In this study the reaction medium consisted of more than 10.000 subvolumes. This was orders of magnitude higher than the number of the reaction and diffusion events that could occur within the system, which was only on the order of 10 . Hence, choosing the subvolume and not the reaction or diffusion event clearly is the time consuming step. To strengthen our argumentation even further, we have also compared the efficiency of the algorithm when simulating a reaction diffusion system with a larger number of chemical species and reactions. For this we used the PTS reaction system, which we will present in some detail in chapter 5 . When simulating this system one has to choose from twenty eight different reaction and diffusion events. As expected, this increase in the number of reaction and diffusion events did not alter our overall result as the Logarithmic Classes Method still outperformed the Next Subvolume Method.

\subsubsection{Improved version of the Logarithmic Classes Method}

The analysis and comparison of the algorithms in the previous sections has revealed that the Logarithmic Classes Method is superior to the other two Monte Carlo methods. However, implementing and using the Logarithmic Classes Method as it was described in the second chapter is not optimal as it is possible to increase the efficiency of this algorithm even further. Most importantly, the LCM can be optimised by adopting the idea of [Cao et al., 2004]: Since the number of the logarithmic classes is generally low, we can order them according to their reactivities. If one now subtracts the largest reactivities first, one increases the likelihood of choosing the correct logarithmic class in one of the first steps. Clearly, this is an improvement over the naive linear selection method used so far, in which the reactivities are subtracted by always starting with the lowest logarithmic class. However, when using the proposed improvement, one needs to keep track of whether the order of the logarithmic classes is still correct or not. This is easily accomplished by either sorting the logarithmic classes on-line or by calling the quick sort algorithm after the system has been propagated a certain number of times.

Moreover, one could increase the efficiency of all algorithms, if a more sophisticated mechanism for the selection of a reaction or diffusion event was used, once a subvolume 
has been picked. Furthermore, the use of Hash tables for the calculation of the reaction and diffusion weights would also speed up the performance of all the three algorithms. Finally it should also be noted that an increase in performance can be accomplished by employing a different kind of random number generator. So far, we have generated random numbers with the help of the ran2 method from the Numerical Recipes [Press et al., 2002]. This is a linear congruential method which is known to provide uncorrelated random numbers. As opposed to this method a linear shift register random number generator is a lot more efficient. However, this method introduces long range correlations among the random numbers which can corrupt the results of Monte Carlo simulations under certain circumstances [Ferrenberg et al., 1992]. Although it is therefore generally advised against the use of this random number generator, we could not observe any difference when employing it instead of the linear congruential method. When accounting for these changes the improved version of this algorithm takes on the form as depicted in the recipe box below. 


\section{Recipe Improved Logarithmic Classes Method}

1. Initialisation: Calculate propensities of all reaction and diffusion events and calculate weights of all subvolumes. Calculate global weight and set up logarithmic classes table. Sort logarithmic classes according to their weights, such that the class with the highest reactivity stays in the first position and the class with the lowest reactivity is in the back.

2. Determine $\tau$ and set global time $t$ to $t=t+\tau$.

3. Select a logarithmic class $l$.

- Draw a normalised uniformly distributed random number $r$ and set $X=r$ and set $i=-1$

- while $(x>0)$

* Set $i=i+1$

$* X=X-\Lambda_{i}$

* If $i>0$ and $\Lambda_{i}>\Lambda_{i-1}$ then exchange the two classes

4. Select a subvolume form the chosen logarithmic class $\Lambda_{i}$ with the help of the von Neumann rejection method:

- Select randomly a subvolume $j$ from the chosen logarithmic class $\Lambda_{i}$

- Draw a normalised uniformly distributed random number $r_{2}$. If $r_{2} 2^{l+1} \leq$ $\Lambda_{i}$, subvolume $j$ is selected. Else repeat from step 4 .

5. Select an event to be executed within subvolume $j$ and execute it.

6. Update affected subvolumes and weights. Repeat from 2. until the stopping criteria is met. 
48 CHAPTER 3. VARIATION 1: COMPARING THE EFFICIENCY OF THE DIFFERENT APPROACHES 


\section{Variation 2: Monte Carlo Simulation of lateral signal propagation}

in which we investigate whether chemical wave fronts provide a reliable mechanism for long range chemical signalling. Starting with two analytical models for the spreading of phophorylation in the EGF receptor system, we investigate how this bistable system is influenced by intrinsic and spatial noise. Noise leads to long-tailed distributions which may cause the spontaneous switching between the steady states. Moreover we will calibrate the spatial noise caused by molecular crowding with experimental data. Finally we show that a chemical wave always propagates with constant velocity through an inhomogeneous medium. However, at the same time its interface disperses which limits the long range signalling capabilities of this mechanism.

4.1 Introduction ................... 49

4.2 Lateral signal propagation . . . . . . . . . . . . . 51

4.2.1 Experimental finding . . . . . . . . . . . . . 51

4.2 .2 Current Model . . . . . . . . . . . . . . . . 53

4.3 Extending the current model . . . . . . . . . . 56

4.3.1 Mathematical modelling . . . . . . . . . . . . 56

4.3.2 Computational Modelling . . . . . . . . . . . 60

4.4 Molecular crowding . . . . . . . . . . . . . . . . 68

4.4.1 Lateral signal propagation in the presence of molecular

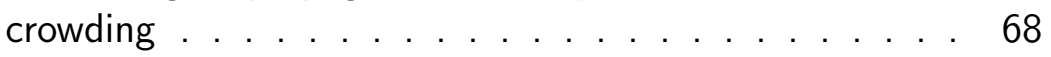

4.4.2 Chemical wave fronts in an inhomogeneous medium . . 71

\subsection{Introduction}

After having presented, compared and extended Monte Carlo algorithms for the simulation of reaction diffusion systems we will now turn to modelling chemical signal transduction cascades. Of course we will employ the previously presented Monte Carlo methods in the course of this chapter, in which we deal with the propagation of chemical information over long distances. Overall, the spatial transport of information within chemical signalling 
cascades is poorly understood in physical terms. In some cases reactant molecules of a chemical signalling pathway could be fluorescently marked and it could be shown that they perform simple diffusional motion [Schwille et al., 1999]. At the same time it is however obvious that diffusion can not be the only mechanism for transporting information over intracellular length scales ranging from $1 \mu \mathrm{m}$ to $1 \mathrm{~m}$. The transport times would become prohibitively long for distances beyond a few $\mu \mathrm{m}$ since the distance $s$ covered by a diffusion process scales with the square root of time, i.e. $s \simeq \sqrt{D t}$. In biological environments the diffusion coefficient $D$ typically takes on values of the order of $\mu m^{2} s^{-1}$. A typical protein has a diffusion coefficient of 10 to $50 \mu \mathrm{m}^{2} \mathrm{~s}^{-1}$. Hence it can cross an eukaryotic cell with a diameter of $10 \mu \mathrm{m}$ in 2 to $10 \mathrm{~s}$, but it already needs between 200 and $1000 \mathrm{~s}$ to cover a cell with a diameter of $100 \mu \mathrm{m}$.

The best studied way for effectively transporting a signal over long distances is the active transport mechanism. This way of transporting a signal works by first packing the relevant signalling molecules into vesicles which are subsequently transported by motorproteins along the microtubules of the cell's cytoskeleton [Alberts et al., 1994]. However, in the last years evidence has been gathered in the field of survival signalling in neuronal cultures that active transport can not be the sole mechanism responsible for long range chemical signalling. In particular in the cell bodies of neurons, it has been observed that the phosphorylation levels of the nerve growth factor receptors TrkA are elevated just one minute after the application of the receptor's ligand to the nerve terminal, which can lie several millimetres away from the soma [Macinnis and Campenot, 2002]. Since this elevation occurs much faster than one would expect, if the signal was propagated by an active transport mechanism, it has been hypothesised that chemical waves might be responsible for the transport [Ginty and Segal, 2002]. Overall, chemical waves have been observed in various different context before and examples from cell biology include the development of Zebrafish eggs [Lee et al., 1999] or the aggregation of Dictyostelium amoebae [Levine et al., 1996]. Furthermore, within the context of survival signalling there exists at least one well studied example of signal propagation by chemical wave fronts, i.e. the lateral spreading of epidermal growth factor (EGF) receptor phosphorylation levels on the plasma membrane [Verveer et al., 2000].

In this chapter we will deal with the lateral spreading of EGF receptor phosphorylation as a model system for long range signal propagation by chemical wave fronts. Apart from providing a prototypical system for long range chemical signalling, the EGF receptor system is also interesting because it plays an important role in development. Moreover its dysfunction can be the cause of cancer [Wiley et al., 2003]. This finding has made the EGF receptor system a target for drugs that were developed for treating this disease. Overall this chapter is organised as follows: First we will review the experimental findings along with a kinetic model which was proposed for explaining the observations. Subsequently we will extend this model by incorporating space. In particular we will focus on the differences arising due to a two or three dimensional representation of space. Furthermore we will also discuss how noise and molecular crowding affects the system under investigation. 

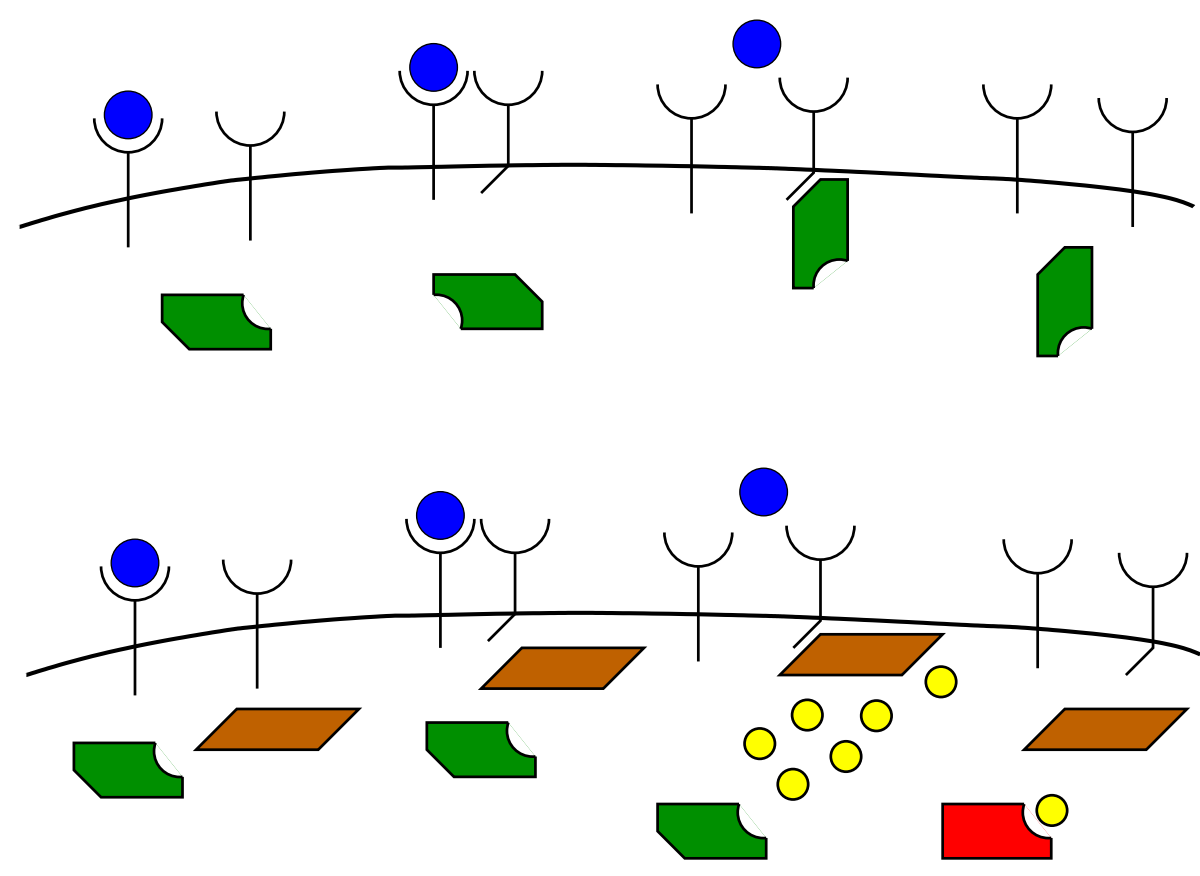

Figure 4.1: Cartoon representation of the EGF receptor system. The upper row shows two EGF receptor forming a dimer, which leads to the phosphorylation of one of the receptors. Subsequently this receptor is dephosphorylated by an active phosphatase molecule. The lower row depicts the scenario in which the phosphorylated receptor activates the NADPH oxidase, which leads to the production of hydrogen peroxidase molecules. These particles are able to deactivate the phosphatase molecules so that the receptor remains phosphorylated. In the plot the blue circles account for the ligand and dephosphorylated receptor have a straight intracellular side whereas phosphorylated receptors are bend. Active phosphatase molecules are green, where the deactivated phosphatases are coloured in red. The NADH oxidase is given by the brown shape whereas the hydrogen peroxidase molecules have the form of the yellow circles.

\subsection{Lateral signal propagation}

\subsubsection{Experimental finding}

The existence of ligand independent waves of EGF receptor activation was first discovered by [Verveer et al., 2000]. A very brief introduction to the experimental imaging techniques used in this study is given in the box at the end of this section. The authors of that paper locally activated a special kind of EGF receptors, namely the ErbB1 receptor, by applying EGF which was covalently attached to beads of approximately $0.8 \mu \mathrm{m}$ in diameter. Since the receptors were fused to the green fluorescent protein GFP, they were able to observe receptor phosphorylation by fluorescence energy transfer (FRET). FRET 
occurred between the labelled receptors and an antibody against phosphotyrosine, which was labelled with a cyan dye (CY1). The FRET induced fluorescence lifetime decrease was observed using fluorescence lifetime imaging (FLIM) and a special analysis of FLIM data allowed for quantitative, spatially resolved mappings of phosphorylated receptors. The observed wave front emanated into all directions from the point of application of the EGF beads and about one minute after local stimulation the entire plasma membrane of the cell contained activated that is phosphorylated receptors. From microscopy, they assured that the beads could be considered as immobile. Hence the author concluded that there must be a potent mechanism by which global phosphorylation of EGF receptors can be achieved. Two years later, the finding of ligand independent waves was independently confirmed in a study by [Sawano et al., 2002]. Furthermore Sawano and coworkers showed that the onset of a wave crucially depends on the number of EGF receptors embedded within the plasma membrane. Clearly, a model for the spreading of activation across the plasma membrane would have to account for such a kind of threshold behaviour. Moreover, from a physiological perspective such a threshold behaviour is very plausible. Especially during development the cell needs to be able to distinguish between the random activation of a single receptor and the real signal caused by the presence of EGF in the extracellular medium.

In 2003, Reynolds and coworkers conducted a set of experiments which lead to the proposal of a minimal reaction network capable of explaining the lateral spreading of receptor phosphorylation within the EGF receptor system [Reynolds et al., 2003]. We tried to visualise their findings in the cartoon model given in Figure 4.1. The starting point of their model is the finding that EGF receptors are capable of transiently forming dimers. When dimerised, the receptors can transphosphorylate each other on tyrosine residues in the intracellular part of the receptor. Upon the binding of EGF this process is facilitated such that the autophosphorylation reaction proceeds with a much higher rate. Overall, the authors therefore showed that EGF receptors are able to activate each other with the help of an autocatalytic reaction. Furthermore, the authors could show that phosphorylated EGF receptors are capable of inhibiting protein tyrosine phosphatase (PTP) molecules. In the resting condition, in which no EGF signal is present, these molecules are responsible for keeping the concentration of active EGF receptors at a low value (depicted in the upper part of Figure 4.1). The inhibition exerted by EGF receptors upon PTP is most likely accomplished through hydrogen peroxidase. These fast diffusing molecules are rapidly produced by nicotinamide adenine dinucleotide phosphate (NADPH) oxidase which in return is activated after the stimulation and phosphorylation of EGF receptors. This part of the signalling cascade is shown in the lower part of Figure 4.1. According to this picture, a strong stimulation of the EGF receptors leads to a sufficient elevation of hydrogen peroxidase so that PTP is no longer capable of dephosphorylating the active EGF receptors. Hence, EGF activation is coupled with the inhibition of PTP, which ultimately leads to an even stronger phosphorylation of EGF receptors on the plasma membrane. Overall, this positive feedback loop can therefore lead to the propagation of 
receptor phosphorylation across the plasma membrane.

\section{Experimental Techniques: FRET and FLIM}

Fluorescence resonance energy transfer (FRET) can be observed between two fluorescent dyes. The general principle of FRET is depicted in the plot below. Suppose that we are given two dyes, the green fluorescent protein GFP and the cyan fluorescent protein CFP. If GFP is selectively excited by a laser, a transfer of energy from the GFP to the CFP molecule can occur. Consequently, CFP can emit a photon although it has not been excited by a laser. Since the efficiency of FRET is strongly dependant on the distance between the two molecules, in fact the FRET efficiency decreases as the sixth power of the distance between the two molecules, it is a widely used tool in biochemistry for the detection of conformational changes and protein protein interactions.

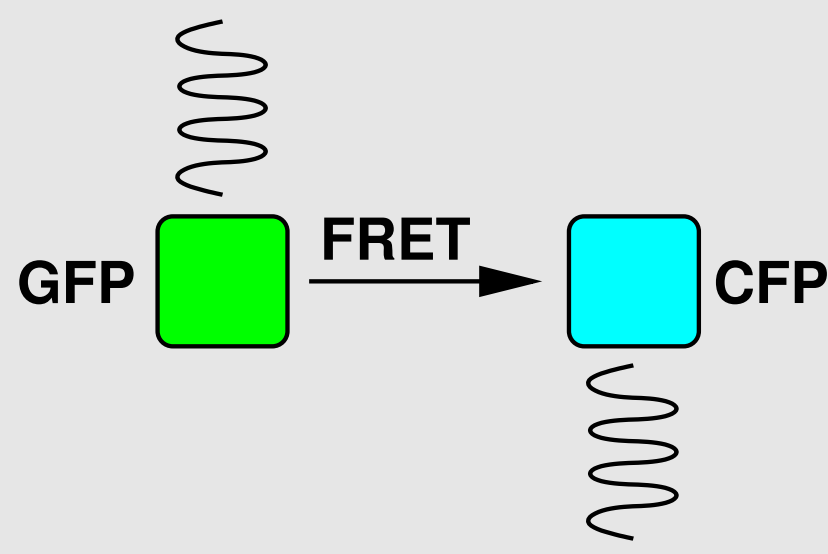

FLIM stands for fluorescence lifetime imaging and is a technique with which one can produce images in which each pixel codes for the rate with which the fluorescence at that point decays with respect to time. Since fluorescence lifetimes of both the acceptor and donor are changed when FRET occurs, FLIM allows for a spatially resolved mapping of where in the sample FRET occurs.

\subsubsection{Current Model}

As a starting point for our investigation of lateral signal propagation in the EGF receptor system we consider the model proposed by [Reynolds et al., 2003], which is depicted in the block diagram in Figure 4.2. This model only accounts for the two key molecules, i.e. it accounts for the receptor tyrosine kinase and for the protein tyrosine phosphatase but neglects the NADPH and the hydrogen peroxidase molecules. The receptors exists in either the native (RTK) or the phosphorylated (RTKp) state and the phosphatase 


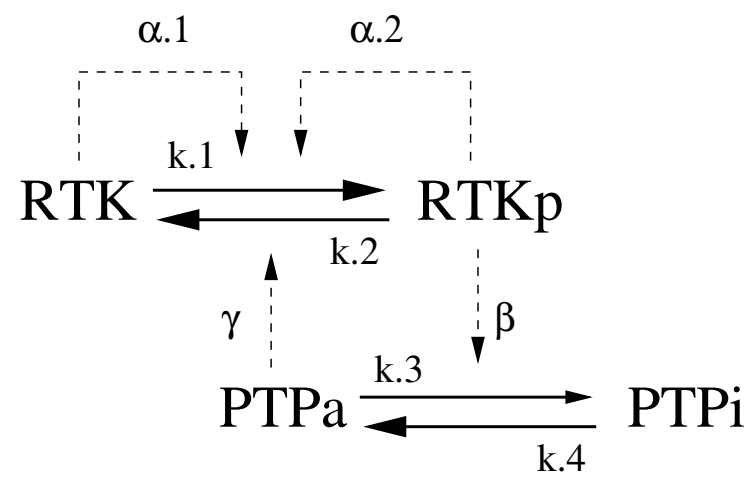

Figure 4.2: Model proposed by [Reynolds et al., 2003] for lateral signal propagation

molecules are either active (PTPa) or inactive (PTPi). Notice, that the model does not differentiate between monomeric receptors and dimers and that it also does not include receptor phosphatase complexes. In their active state, phosphatase molecules are capable of dephosphorylating phosphorylated EGF receptors with rate $k_{2} \gamma$. RTKp molecules in return deactivate active phosphatases with rate $k_{3} \beta$. Furthermore inactive phosphatase molecules are reactivated with rate $k_{4}$, while the phosphorylation of the receptors either occurs with rate $k_{1} \alpha_{1}$ or $k_{1} \alpha_{2}$. When introducing the variables $n$ and $c$ for the concentrations of RTKp molecules and PTPa molecules as well as $n_{0}$ and $c_{0}$ for the total concentration of the respective molecules, Figure 4.2 gives rise to the following two differential equations describing the dynamics of the system:

$$
\begin{aligned}
\partial_{t} n & =\left(n_{0}-n\right)\left(k_{1} \alpha_{1}\left(n_{0}-n\right)+k_{1} \alpha_{2} n\right)-k_{2} \gamma n c \\
\partial_{t} c & =k_{4}\left(c_{0}-c\right)-k_{3} \beta n c .
\end{aligned}
$$

After rescaling these two equations with the help of the following substitutions

$$
\begin{array}{llr}
u=n / n_{0}, & v=c / c_{0}, & \tau=n_{0} k_{1} \alpha_{1} t, \\
\frac{P}{K}=\frac{k_{2} \gamma c_{0}}{n_{0} k_{1} \alpha_{1}}, & R=k_{4}, & I=k_{3} \beta n_{0},
\end{array}
$$

we arrive at the two equations:

$$
\begin{aligned}
\partial_{\tau} u & =(1-u)\left[(1-u)+\frac{\alpha_{2}}{\alpha_{1}} u\right]-\frac{P}{K} u v \\
\partial_{\tau} v & =\frac{R}{K}(1-v)-\frac{I}{K} u v .
\end{aligned}
$$

Let us first assume that the dynamics of the phosphatase molecules relax much faster than the dynamics of the kinase molecules. Hence we set the left hand side of equation 


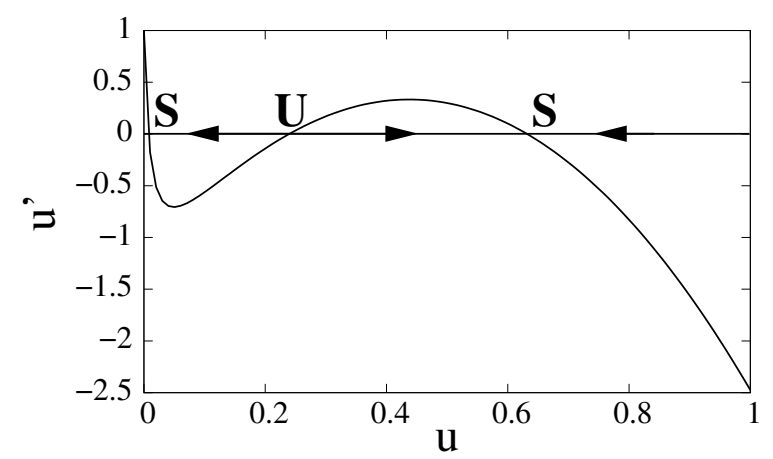

Figure 4.3: A phase plot showing $u^{\prime}$ as a function of $u$ clearly reveals the bistable character of the EGF receptor system.

4.4 to zero, solve for $v$, and insert the solution $v=1 /(1+(I / R) u)$ into the first equation. Note, that our assumption does not necessarily hold true, but since we are only interested in the qualitative behaviour of the system, this can safely be done. Thus we arrive at

$$
\partial_{\tau} u=(1-u)\left[(1-u)+\frac{\alpha_{2}}{\alpha_{1}} u\right]-\frac{P}{K} u \frac{1}{1+\frac{I}{R} u} .
$$

Examining the phase plot of this equation with parameters $\alpha_{2} / \alpha_{1}=10, I / R=100$, and $P / K=250$ reveals that the system behaves bistable, i.e. there are two stable steady states in which the system normally resides. These two fixpoints are denoted as the uninduced and the persistently active state. In the uninduced state virtually no receptors are phosphorylated whereas a majority of receptors is phosphorylated in the persistently active state. Both states are separated by an unstable fixpoint such that there exists a threshold value in the concentration of phosphorylated EGF receptors which needs to be surpassed in order to switch from the uninduced to the persistently active state. When evaluating equation 4.5 numerically with different sets of parameters, one notices that the exact position of the threshold depends on several parameters. Decreasing the ratio of phosphatase to kinase activity $(P / K)$ while leaving the other parameters constant will for example decrease the value of the unstable fixpoint so that switching from the uninduced to the persistently active state is facilitated. In contrast to that a decrease in the ratio of phosphatase inactivation to phosphatase reactivation $(I / R)$ will raise the value for the threshold. In their paper Reynolds et al. [2003] could support these model predictions with the help of experiments. Furthermore they could also explain the finding of [Sawano et al., 2002] that the number of expressed EGF receptors crucially effects whether a wave is induced or not. 


\subsection{Extending the current model}

Although the model of [Reynolds et al., 2003] correctly captures the threshold behaviour of the EGF receptor system, it comes with the drawback that it totally neglects space and diffusion of the involved molecules. We will therefore begin by extending the model and remove the assumption of a well stirred medium. Since the protein tyrosine phosphatase responsible for dephosphorylating the EGF receptors has not yet been identified in this system, we will consider two different scenarios. In the first scenario both the receptors as well as the phosphatases will be localised in the plasma membrane of the cell. The second case will be different in that the PTP will reside within the cytosol, where it is reactivated once it has been deactivated by the receptor tryrosine kinase at the plasma membrane. Note, that both scenarios are equally plausible from a physiological perspective [Östman and Böhmer, 2001].

In the following, we will first use an analytical calculation to investigate how this difference in localisation affects the steady states of the system. Subsequently we will use a computational approach to study this system in greater detail. In the last section of this chapter we will further use the computational approach in order to examine how molecular crowding is going to affect the overall behaviour of the system.

\subsubsection{Mathematical modelling}

We begin our mathematical analysis for the case in which both EGF receptors and phosphatase molecules are located on the plasma membrane. In the steady state, both chemical species are homogeneously distributed. In principle this corresponds to the well stirred approach used in the original model. Consequently the steady state concentrations can easily be obtained by setting $\partial_{\tau} u$ and $\partial_{\tau} v$ in equations (4.3) and (4.4) to zero. Obviously the EGF receptor's steady state concentration $u^{*}$ is given by the solution of the equation:

$$
\left(1-u^{*}\right)\left[\left(1-u^{*}\right)+\frac{\alpha_{2}}{\alpha_{1}} u^{*}\right]-\frac{P}{K} u^{*} v^{*}=0 .
$$

Solving the second equation gives rise to the phosphatase's steady state concentration $v^{*}$. It is given by the expression

$$
v^{*}=\frac{1}{1+\frac{I}{R} u^{*}} .
$$

If we insert this expression for the steady state concentration of the system's phosphatase molecules into equation (4.6), this gives rise to the cubic equation

$u^{3}\left(1-\frac{\alpha_{2}}{\alpha_{1}}\right) \frac{I}{R}+u^{2}\left(1+\frac{\alpha_{2}}{\alpha_{1}}\left(\frac{I}{R}-1\right)-2 \frac{I}{R}\right)+u\left(\frac{\alpha_{2}}{\alpha_{1}}+\frac{I}{R}-\frac{P}{K}-2\right)+1=0$,

in which we have omitted the asterisks for notational convenience. In order for the system to be in the bistable regime, this last equation needs to have three real distinct roots. 
This is the case if the discriminant of the polynome is smaller than zero. Hence, for the system's parameters we have to require that they fulfil the condition

$$
\begin{gathered}
\left(1+\frac{\alpha_{2}}{\alpha_{1}} \frac{I}{R}-\frac{\alpha_{2}}{\alpha_{1}}-2 \frac{I}{R}\right)^{2}\left(\frac{\alpha_{2}}{\alpha_{1}}+\frac{I}{R}-\frac{P}{K}-2\right)^{2}-4\left(\frac{I}{R}-\frac{\alpha_{2}}{\alpha_{1}} \frac{I}{R}\right)\left(\frac{\alpha_{2}}{\alpha_{1}}+\frac{I}{R}-\frac{P}{K}-2\right)^{3}- \\
4\left(1+\frac{\alpha_{2}}{\alpha_{2}} \frac{I}{R}-\frac{\alpha_{2}}{\alpha_{1}}-2 \frac{I}{R}\right)^{3}-27\left(\frac{I}{R}-\frac{\alpha_{2}}{\alpha_{1}} \frac{I}{R}\right)^{2}+ \\
18\left(\frac{I}{R}-\frac{\alpha_{2}}{\alpha_{1}} \frac{I}{R}\right)^{2}\left(1+\frac{\alpha_{2}}{\alpha_{1}} \frac{I}{R}-\frac{\alpha_{2}}{\alpha_{1}}-2 \frac{I}{R}\right)\left(\frac{\alpha_{2}}{\alpha_{1}}+\frac{I}{R}-\frac{P}{K}-2\right)<0
\end{gathered}
$$

With the help of this equation, one can now easily calculate in which range the third parameter has to lie in order for the system to be bistable, if the other two parameters are given. For instance, if we choose $\alpha_{2} / \alpha_{1}=10$ and $I / R=100$, it immediately follows numerically that $P / K$ has to lie approximately within the range between 164 and 284 .

In the following we will now investigate how the steady state concentrations of the two molecular species are changed if the phosphatase is no longer localised in the membrane but in cytosol of the cell instead. When being in the bulk the deactivation of phosphatase molecules is only possible at the membrane, into which the receptor tyrosine kinases are embedded. As opposed to that the reactivation of the phosphatase can occur anywhere within the cytosol. The starting point of our analysis is therefore given by the three partial differential equations describing the dynamics of the active receptors and the active phosphatases:

$$
\begin{array}{rlrl}
\partial_{t} n-D_{R} \nabla^{2} n & =\left(n_{0}-n\right)\left[k_{1} \alpha_{1}\left(n_{0}-n\right)+k_{2} \alpha_{2} n\right]-k_{2} \gamma n c \\
\partial_{t} c-D_{P} \nabla^{2} c & =k_{4}\left(c_{0}-c\right) & & \text { (in the cytosol) } \\
\partial_{t} c-D_{P} \nabla^{2} c & =-k_{3} \beta n c & & \text { (at the membrane) }
\end{array}
$$

When analysing these equations, we use a three dimensional Cartesian geometry with coordinate axes $x, y$, and $z$. In particular we choose the coordinate system in such a way that the membrane is situated in the $x y$-plane at $z=0$. Before we continue, we again first rescale the system. For this the same substitutions as given in the previous section are used along with the two replacements $D_{u}=\frac{D_{R}}{k_{1} \alpha_{1} n_{0}}$ and $D_{v}=\frac{D_{P}}{k_{1} \alpha_{1} n_{0}}$. Consequently, we arrive at the two dimensionless equations

$$
\begin{aligned}
\partial_{\tau} u-D_{u} \nabla^{2} u & =(1-u)\left[(1-u)+\frac{\alpha_{2}}{\alpha_{1}} u\right]-\frac{P}{K} u v \\
\partial_{\tau} v-D_{v} \nabla^{2} v & =\frac{R}{K}(1-v)-\delta(z) \frac{I}{K} u v .
\end{aligned}
$$

Note that we have cast the dynamics of the phosphatase molecules in equation 4.13 into a single equation using a delta function. Further notice that diffusion of the phosphatase molecules takes place in the three dimensional space whereas diffusion of the receptors is confined to the membrane. Since it is required for the homogeneous steady state that 
both molecular species are homogeneously distributed (the receptors in the membrane and the phosphatases in the cytosol), the complexity of our problem is reduced. The diffusion term in equation 4.12 can be neglected so that the steady state concentration of the EGF receptors is as in the previous case given by the solution of equation 4.6. However, if one wants to solve this equation, one has to pay attention that $v^{*}$ now stands for the steady state concentration of the phosphatase molecules at position $z=0$. For calculating this value we begin by setting $\partial_{\tau} v=0$ in equation 4.13 . Thus we obtain an ordinary differential equation with which we are able to calculate the steady state concentration of the phosphatase molecules as a function of $z$. For solving this differential equation, we have to specify two boundary conditions. For the first boundary condition, it is reasonable to assume that the number of inactivated phosphatase molecules vanishes as the distance to the membrane becomes increasingly large because phosphatase molecules can only be deactivated at the membrane and are reactivated in the cytosol. Consequently one should encounter only active phosphatase molecules, if one is infinitely far away from the membrane. Hence the first boundary condition is given by

$$
\lim _{z \rightarrow \infty} v(z)=1
$$

The second boundary condition is obtained by noting that the membrane is a sink for the active phosphatase molecules. In the steady state, the diffusive flux of active phosphatase molecules into the membrane must be equal to their rate of inactivation. This follows immediately from mass conservation. We therefore have our second boundary conditions, which reads

$$
D_{v} \partial_{z} v^{*}(z=0)=-\frac{I}{K} u^{*} v^{*}
$$

Hence, for calculating the active protein phosphatase's steady state concentration profile $v^{*}(z)$, we have to solve the equation

$$
-D_{v} \frac{\partial^{2} v^{*}}{\partial z^{2}}=\frac{R}{K}\left(1-v^{*}\right)
$$

subject to the two boundary conditions given by equations 4.14 and 4.15 . This can be done in a straightforward manner, if one uses the coordinate transformation $y=v^{*}-1$. After carrying out the calculations, one arrives at the solution

$$
v^{*}(z)=1-\frac{I}{\sqrt{K R D_{v}}} u^{*} v_{0}^{*} \exp \left\{-\sqrt{\frac{R}{K D_{v}}} z\right\} .
$$

When setting $z$ to zero, one obtains the steady state phosphatase concentration $v_{0}^{*}$ at the membrane, which is given by

$$
v_{0}^{*}=\frac{1}{1+\frac{I}{\sqrt{K R D_{v}}} u^{*}} .
$$


If we now compare the two steady state concentrations of the phosphatase molecules at the plasma membrane (equation 4.7 and 4.18), one notices that this value depends in both equations on the ratio of the rate of phosphatase inactivation $(I)$ to the rate of phosphatase reactivation. When the phosphatase is localised in the plasma membrane, the reactivation rate is given by $R_{2 D}=R=k_{4}$ because it only depends on the chemical reaction $\mathrm{PTPi} \rightarrow \mathrm{PTPa}$. In the case that the phosphatase is distributed throughout the cytosol, the reactivation of phosphatase molecules close to the membrane also depends on the diffusive exchange of inactive molecules with active molecules from the bulk. Hence, in the three dimensional system the reactivation rate is given by $R_{3 D}=\sqrt{K R D_{v}}=\sqrt{D_{P} k_{4}}$. Consequently $R_{2 D}$ and $R_{3 D}$ are related as $R_{2 D} / R_{3 D}=\sqrt{k_{4} / D_{P}}$.

To start the discussion of our model we notice that the main consequence of localising the phosphatase molecules in the cytosol lies in the fact that the homogeneous steady states of the phosphorylated receptor molecules now depend on the flux of the phosphatase molecules. In particular this implies that the receptor molecule dynamics are a function of the difference between the concentration of active phosphatase molecules at the membrane and in the inside of the cell. However, since this difference obviously depends on the total phosphatase concentration $c_{0}$, this provides a starting point for experimentalists to test whether the phosphatase molecules regulating the EGF receptors are embedded in the membrane or reside in the cytosol of the cell. Namely by reducing the concentration of phosphatase molecules in the cytosol, they should be able to change the behaviour of a cell. If our model is correct, it should be possible to push a cell from the bistable regime into the persistently active state when selectively blocking the phosphatase molecules in the inside of the cell.

To complete this first discussion of our model we investigate whether the system exhibits bistability when choosing physiologically plausible parameters. From a mathematical perspective the only difference between the original and our model is given by the rate according to which the phosphatase molecules are reactivated. Hence, we can mostly use the same parameters as [Reynolds et al., 2003]. Therefore we choose $\alpha_{2} / \alpha_{1}=10$ for the activation rates of the receptors and we set the ratio of maximal phosphatase to maximal kinase activity to $P / K=250$. The main task now consists of checking whether we can find physiologically plausible parameters, so that the quotient of maximal phosphatase inhibition and the rate of phosphatase reactivation $I / R_{3 D}=k_{3} \beta n_{0} / \sqrt{k_{4} D_{p}}$ will be equal to 100 . This is the value which was used in [Reynolds et al., 2003]. When selecting the rates, one has to further pay attention that the concentration of active phosphatase molecules in the cytosol reverts on plausible length scale to its resting value $c_{0}$. According to equation 4.17, the gradients will be steep for the case that $k_{4} / D_{p}>1$. Consequently we choose $k_{3} \beta n_{0}=200 \mu \mathrm{m} \mathrm{sec}-1, k_{4}=4 \mathrm{~s}^{-1}$ and $D_{p}=1 \mu \mathrm{m}^{2} \mathrm{~s}^{-1}$, such that we have $I / R_{3 D}=100$. As seen before the system will be in the bistable regime with this set of parameters. Note that the chosen values for $D_{p}$ and $k_{4}$ are in accordance with typical values for the diffusion coefficient of cytosolic proteins and the first order rate constants accounting for phosphorylation and dephosphorylation reactions. The former 


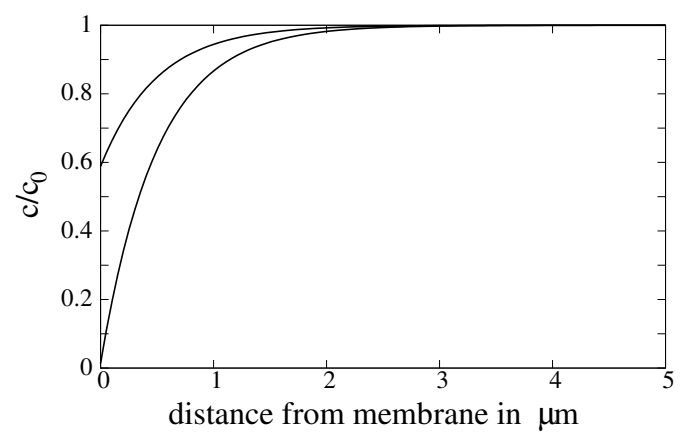

Figure 4.4: Gradients of the active phosphatase concentration in the uninduced and persistently active state when choosing the system's parameters as described in the main text.

normally takes on values between 1 and $50 \mu \mathrm{m}^{2} \mathrm{~s}^{-1}$, whereas typical values for the latter range from 0.1 to $100 \mathrm{~s}^{-1}$ [Kholodenko et al., 2000]. Moreover, with these parameters the fraction of phosphorylated EGF receptors in the membrane will be equal to approximately 0.007 and 0.632 in the uninduced and the persistently active state, whereas the fraction of active phosphatase molecules at the membrane will be equal to approximately 0.588 and 0.0156 . As can be seen in Figure 4.4, with this choice of the parameters, it takes around $2 \mu \mathrm{m}$ to reactivate all phosphatase molecules. This is a plausible value, if one assumes that a typical eukaryotic cell is spherical and has a diameter of $10 \mu \mathrm{m}$. Overall we therefore conclude that our model is capable of reproducing bistability for a physiologically realistic set of parameters.

\subsubsection{Computational Modelling}

Up to now, we have analytically calculated the homogeneous steady states of the EGF receptor system for the two different scenarios in which the phosphatase molecules are either embedded in the plasma membrane or situated in the cytosol of the cell. In this section we will now analyse the role of noise in both the two- as well as the three-dimensional system. For this task we will employ the Monte Carlo methods which we presented in the previous chapters. Furthermore we will show in the course of this section that EGF receptor phosphorylation is also in the three dimensional system spread by a propagating wave front.

When simulating the two dimensional system, in which both the EGF receptors as well as the phosphatase molecules are embedded in the membrane, we use an area of $4 \times 4 \mu m^{2}$ as a reaction medium, which we discretise into 4096 reaction cells. When simulating the three dimensional EGF receptor system, we use a cubical reaction medium with a sidelength of $4 \mu \mathrm{m}$. One side of the cube is identified as the membrane in which 

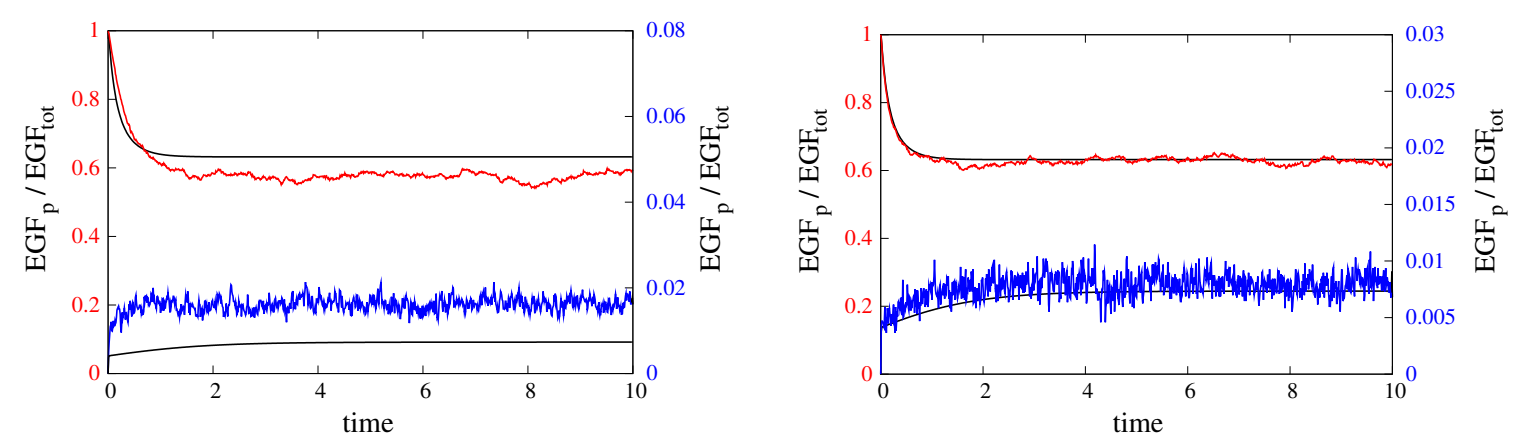

Figure 4.5: Left: Three dimensional system. Right: Two dimensional system. Depending on the initial condition the fraction of phosphorylated EGF receptors settles in both system to the two different homogeneous steady states. The scale for the red trajectory is given on the left $y$-axis whereas the scale for the blue trajectories is given on the right $y$-axis. The solid black lines is the deterministic solution obtained by numerically integrating the chemical rate equations.

the receptor molecules reside. To reduce the number of possible diffusion events which increases the overall computational efficiency, the reaction medium is discretised in such a way that the volumes of the gridcells vary. In particular the gridcells close the membrane have small volumes. The size of the subvolumes subsequently increases the further distant they are situated from the membrane. A detailed description of how the diffusion rates are calculated on such a grid is given in the fifth chapter. Special attention needs to be drawn to the units of the involved chemical concentrations and the rate constants. As the receptors are situated in the membrane, the concentrations of RTK and RTKp are given in units of $\mu M \mu m$, whereas the concentrations of PTPa and PTPi are given in units of $\mu M$ because they live in the cytosol. Since the employed Monte Carlo approach only uses three dimensional voxels, we have to convert the surface concentrations of the two isoforms of the receptor tyrosine kinase into volume concentrations. This is easily accomplished by multiplying the surface concentrations with the surface to volume ratio. As the rate constants for the reactions among receptor molecules are given in units of $\mu M^{-1} \mu m^{-1} s^{-1}$, they need to be converted into $\mu M^{-1} s^{-1}$, which is achieved by dividing these rates with the surface to volume ratio.

In the first simulation we want to investigate whether the two systems still exhibit two distinct homogeneous steady states when stochasticity is accounted for. According to the mathematical analysis the system is bistable, if the reaction rates are chosen in such a way that they give rise to the following dimensionless parameters: $\alpha_{2} / \alpha_{1}=10, P / K=250$, and $I / R=100$. The left plot in Figure 4.5 shows the results of the stochastic simulation in the three dimensional system, whereas the trajectories of the stochastic simulation of the two dimensional system are given in the right plot. In both plots we have also included 
the deterministic trajectories, which are obtained by numerically integrating the chemical rate equations. When analysing Figure 4.5 one basically sees two things. First and most importantly both system are bistable. Depending on the initial condition, the system settles to either one of the two different steady states. When starting the simulation with all receptor molecules being phosphorylated and all phosphatase molecules being deactivated, the system reaches the persistently active state (red trajectories). As opposed to that the system will stay in the uninduced state, if one starts the simulation in a state in which all phosphatase molecules are active and all receptors are in the unphosphorylated state (blue trajectories). Furthermore, we also see that the trajectories of the two dimensional system are well approximated by the deterministic results, which is not the case for the three dimensional system although the same dimensionless parameters were used. That the trajectories of the two dimensional stochastic system are well approximated by the solutions of the chemical rate equations is not surprising as the particle numbers in this simulation are very high. In particular we used values of $1 \mu M \mu m$ and $10 \mu M \mu m$ for the total concentrations of receptor and phosphatase molecules so that 9600 receptors and 96000 phosphatase molecules are embedded in the model membrane. Furthermore the two dimensional system is spatially homogeneous and both molecular species have the same diffusion coefficients. Taken together, these are all major requirements that need to be fulfilled in order for the chemical rate equation to yield a good approximation to the real system. In the three dimensional system these requirements are not met, i.e. the system is spatially inhomogeneous and the particle concentrations are low. For the phosphatase concentration we chose a value of $10 \mu M$ which means that we have 384000 molecules in the reaction medium. Furthermore the concentration of phosphatase molecules exhibits large spatial fluctuations, which becomes obvious when looking at the results depicted in Figure 4.6. In this plot, we have visualised the gradients in the active phosphatase concentration in the $z$-direction. The upper and lower trajectories account for the standard deviation in the active phosphatase concentration in each layer parallel to the membrane. Obviously, the particles are inhomogeneously distributed. Consequently in addition to the particle flux in $z$-direction, fluxes within each layer build up. Both fluxes are not accounted for in a chemical rate equation description and they also do not exist in the two dimensional system. Hence, the large differences between the two simulation results arise. To validate our argumentation, we ran two additional simulations. In the first simulation we increased the concentration of phosphatase molecules in the three dimensional simulation by a factor of ten while leaving the relation among the dimensionless parameters unchanged. The resulting trajectories are better approximated by the chemical rate equations as the relative particle fluctuations are not as pronounced as in the original simulation. That horizontal fluctuations substantially influence the results of a stochastic simulation was shown in the second simulation. Here, we changed the two dimensional system in such a way that the diffusion coefficients of the two chemical species now differed by a factor of 50 . The resulting trajectories no longer overlap with the deterministic results but were shifted towards the three dimensional simulation results. 

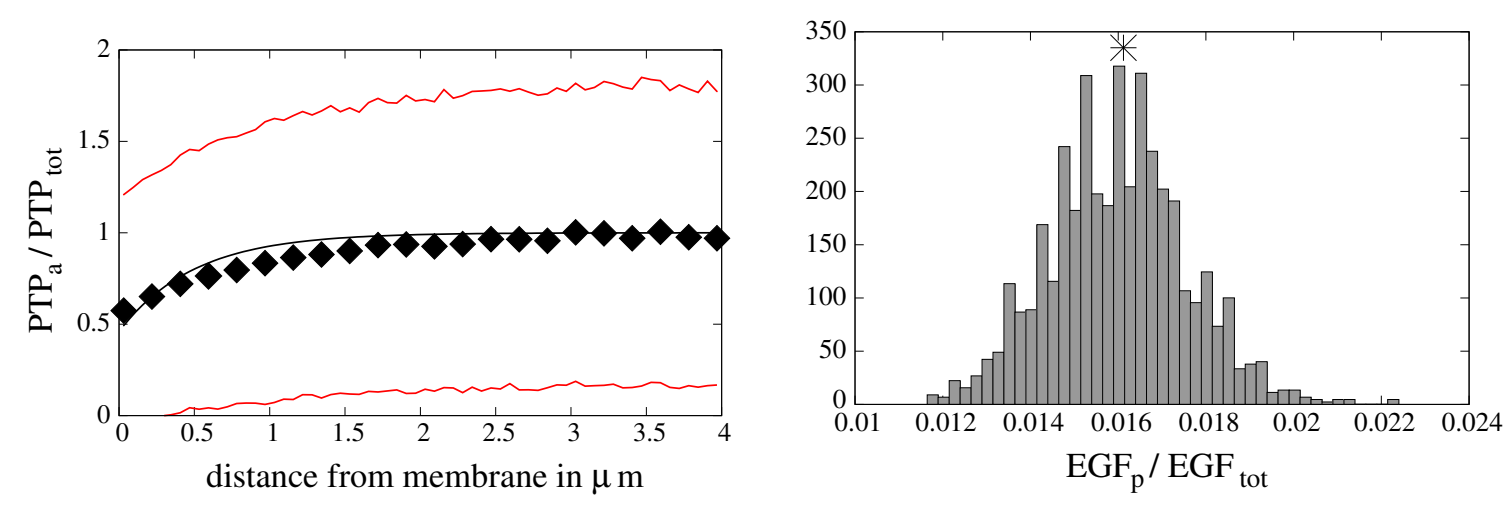

Figure 4.6: Left: Gradient of the active phosphatase concentration in $z$-direction when the system is in the uninduced state. The points account for the mean concentration in a layer parallel to the membrane. The solid black line depicts the theoretical gradient as given by equation (4.17) when using the measured membrane concentration of the active receptor molecules as an input. The two red lines stand for the upper and lower end of the standard deviation around the measured PTPa concentration. Right: Distribution of $\mathrm{EGF}_{\mathrm{a}} / \mathrm{EGF}_{\text {tot }}$ around the steady state value when the system is in the uninduced state. The steady state value of the distribution is indicated by the asterisk. The scale of the $y$-axis is chosen in such a way that the area underneath the histogram is equal to one. Further note that the distribution is slightly skewed to the right.

So far we have seen that the two and the three dimensional system are both bistable in the presence of noise. Apart from that, we have also seen that fluxes arise in the three dimensional system because spatial fluctuations are generally large. Moreover these fluxes cause the three dimensional system to assume different values for the steady state concentration of the phosphorylated EGF receptors than the two dimensional system. However, it is possible to choose the parameters of the two dimensional system in such a way that the same steady state values are reached as in the three dimensional system. When doing so, we find that the distributions of the values around the steady states are also identical; i.e. both distributions have the same width and both distributions are slightly skewed. An example of such a distribution of the $\mathrm{EGF}_{\mathrm{p}} / \mathrm{EGF}_{\text {tot }}$ values around the uninduced state is given in the right plot of Figure 4.6. Interestingly the distributions are skewed to the right in the uninduced state and in the persistently active case they are skewed to the left. Overall this implies that fluctuations preferably occur in the direction of the switch, which causes spontaneous transitions from one state to the other, if these fluctuations are strong enough. In the following we will deal with this phenomenon in greater detail.

We start our discussion by showing a series of snapshots in Figure 4.7 in which the amount of phosphorylated EGF receptors in the membrane is visualised. In this plot we 

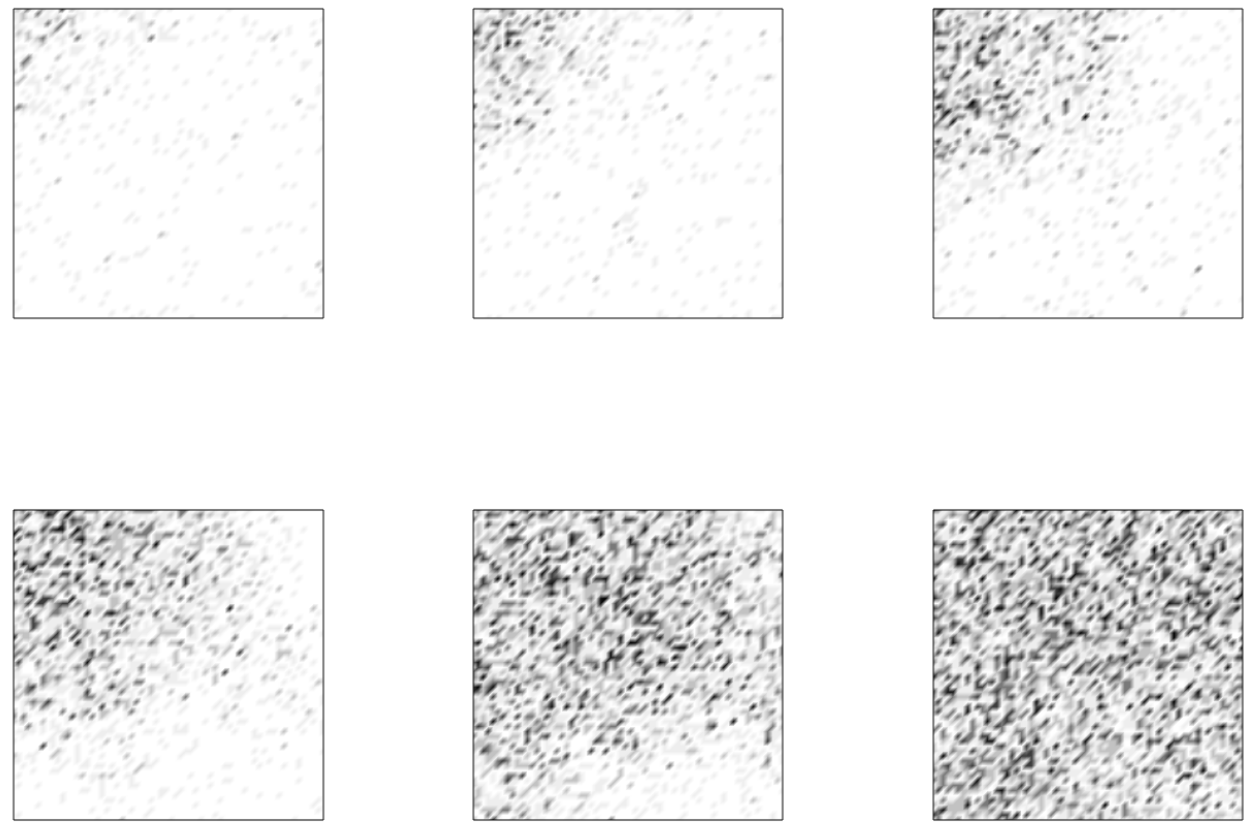

Figure 4.7: Temporal sequence of a patch of membrane showing the spontaneous switching from the uninduced state to the persistently active state. A grey scale coding scheme is used. Dark colours indicate a high proportion of phosphorylated receptors whereas white areas account for the absence of phosphorylated receptors.

have used a grey scale scheme to code for the number of these molecules. Dark levels indicate a high content of phosphorylated receptors whereas light values account for the absence of these particles. The first snapshot shows a local elevation in the number of phosphorylated receptors in the upper left corner of the reaction medium. Subsequently this perturbation builds up and phosphorylation is spread by a chemical wave which propagates through the medium until the entire membrane is in the persistently active state. It is well understood that the probability for the occurrence of a spontaneous switching event depends on the height and width of the barrier separating the uninduced from the persistently active state as well as on the level of noise in the system. In the following we therefore want to quantify the level of noise in the system and set it in relation to the probability with which a spontaneous switching event occurs. For this task we first have to find a method according to which we can define the level of noise in the system. One possibility is to proceed as follows: First we measures the standard deviation with which the concentration of the phosphorylated EGF receptors fluctuates around the uninduced steady state value. Subsequently we rate this value by dividing it by the concentration of phosphorylated EGF receptors that has to be added to the system in order to switch from 


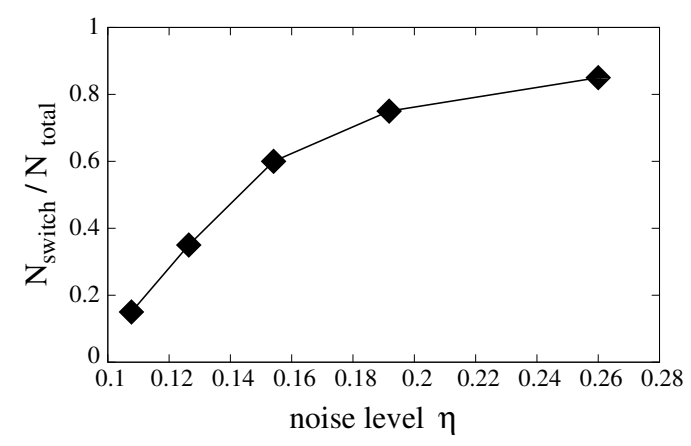

Figure 4.8: Plot visualising how many trajectories switch from the uninduced to the persistently active state as a function of the noise $\eta$ within the system.

the uninduced to the persistently active state. For the deterministic system this concentration difference between the switch value and the uninduced steady state can easily be obtained as it is simply given by difference between the respective roots of equation (4.9). However, when using a stochastic approach we have seen that the deterministic description shows large deviations from the real value, which makes a calculation of this value on the basis of the chemical rate equations infeasible. Consequently we use a numerical approach. We simply start the simulation with different concentrations of phosphorylated EGF receptors embedded in the membrane and observe whether the system settles to the uninduced or to the persistently active state. When doing so we reliably find the threshold concentration needed for calculating the noise in the system. To summarise, the noise level $\eta$ is therefore defined as $\eta=\sqrt{\sigma} /\left(u_{\text {switch }}-u_{\text {uninduced }}\right)$, with sigma accounting for the variance and $u_{\text {switch }}$ and $u_{\text {uninduced }}$ for the concentration of phosphorylated EGF receptors at the switching threshold and in the uninduced steady state. Next we have to calculate how likely it is to observe a stochastic switching event in a certain period of time. This is simply done by simulating for each noise level $\eta$ an ensemble of twenty trajectories. Subsequently we count how often a switching event occured within a time period of $T=15 \tau$. When analysing the results displayed in Figure 4.8, we find that spontaneous stochastic switching events already occur when the fluctuations of the signal are by a factor ten smaller than the difference that needs to be overcome. This is quite remarkable because it further underlines that the distributions around the steady state are not Gaussian. Instead the distribution of the fluctuations around the steady state has a long tail, which causes the spontaneous switching. Furthermore we observe that a spontaneous switching transition already occurs in more than $50 \%$ of the cases, if the noise level $\eta=0.15$ and once the system has exceeded a noise level of 0.25 , virtually all trajectories switch from the uninduced to the persistently active state. Moreover, we observe this behaviour for a parameter range according to which the system should still be bistable, if one only accounted for the mean field deterministic behaviour.

Overall our results clearly demonstrate that the microscopic processes involved in this 
chemical signalling cascade are of a non-Gaussian nature. This leads to distributions of the phosphorylated EGF receptors around their steady state values that are not simply described by their mean and their standard deviation. Instead theses distributions are skewed and have a long tail, which leads to the spontaneous switching behaviour. Ultimately, our results therefore imply that noise in chemical signal transduction cascades can lead to phenotypic variations in a population of identical cells. With the noise having the characteristics as described above, one would observe a bimodal distribution of the cells in a population grown under identical conditions and one portion of the population would be in the persistently active state whereas the other part would be in the uninduced state.

We will end this section on the computational modelling of the EGF receptor system by reproducing in the three dimensional system the experimental results reported in [Verveer et al., 2000]. On the one hand we want to show that a localised perturbation can push the system into the persistently active state. As in the case of spontaneous stochastic switching, the lateral spreading of receptor phosphorylation is mediated by a chemical wave front. More importantly though, our main concern lies on showing that our three dimensional stochastic model is capable of reproducing the experimental findings when using realistic assumptions for the involved parameters. In particular we chose the following parameters for the reaction rates: $\alpha_{2} / \alpha_{1}=10, P / K=25$, and $I / R_{3 D}=110$. Furthermore, we chose $k_{4}=4 \mathrm{~s}^{-1}$ and $D_{P T P}=1 \mu \mathrm{m}^{2} \mathrm{~s}^{-1}$ to obtain sharp gradients in the cytosolic phosphatase concentration. As the diffusive movement of the receptor molecules in the plasma membrane is a lot slower than the movement of the phosphatase molecules in the cytosol, we set $D_{E G F}=0.1 \mu \mathrm{m}^{2} \mathrm{~s}^{-1}$. Notice that all parameters lie within physiologically realistic bounds. With this set of parameters the system will settle to the uninduced state if we start the simulation with an initial condition in which a concentration of $10 \mu M$ of active phosphatase molecules is homogeneously distributed in the cytosol and $1 \mu M \mu m$ of unphosphorylated EGF receptors are embedded in the plasma membrane. In the experiments, the uninduced state was perturbed by permanently exposing the receptors in well defined membrane patches to their endogenous ligand. Technically this was accomplished by exposing the cell to beads to which epidermal growth factor molecules were covalently linked. The diameter of these beads was equal to $0.8 \mu \mathrm{m}$. Control experiments revealed that the EGF receptors are tightly bound to the beads. Furthermore, it has been argued that the presentation of a ligand on a solid substrate blocks endocytosis which leads to a local increase in the receptor density [Sawano et al., 2002]. In order to model the application of EGF linked to beads, we therefore locally introduced a fifth chemical species, which behaves like a persistently phosphorylated receptor; i.e. molecules of this species phosphorylate other EGF receptors and deactivates phosphatase molecules with the same rates as the native phosphorylated EGF receptor. Overall, we introduced a concentration of $10 \mu M \mu m$ of this new species to a square region in the middle of the reaction medium which had a size of $0.88 \mu \mathrm{m}^{2}$. Snapshots showing the degree of phosphorylation in the membrane at different times are obtained by averaging over an ensemble of ten simulations. The results are displayed in 

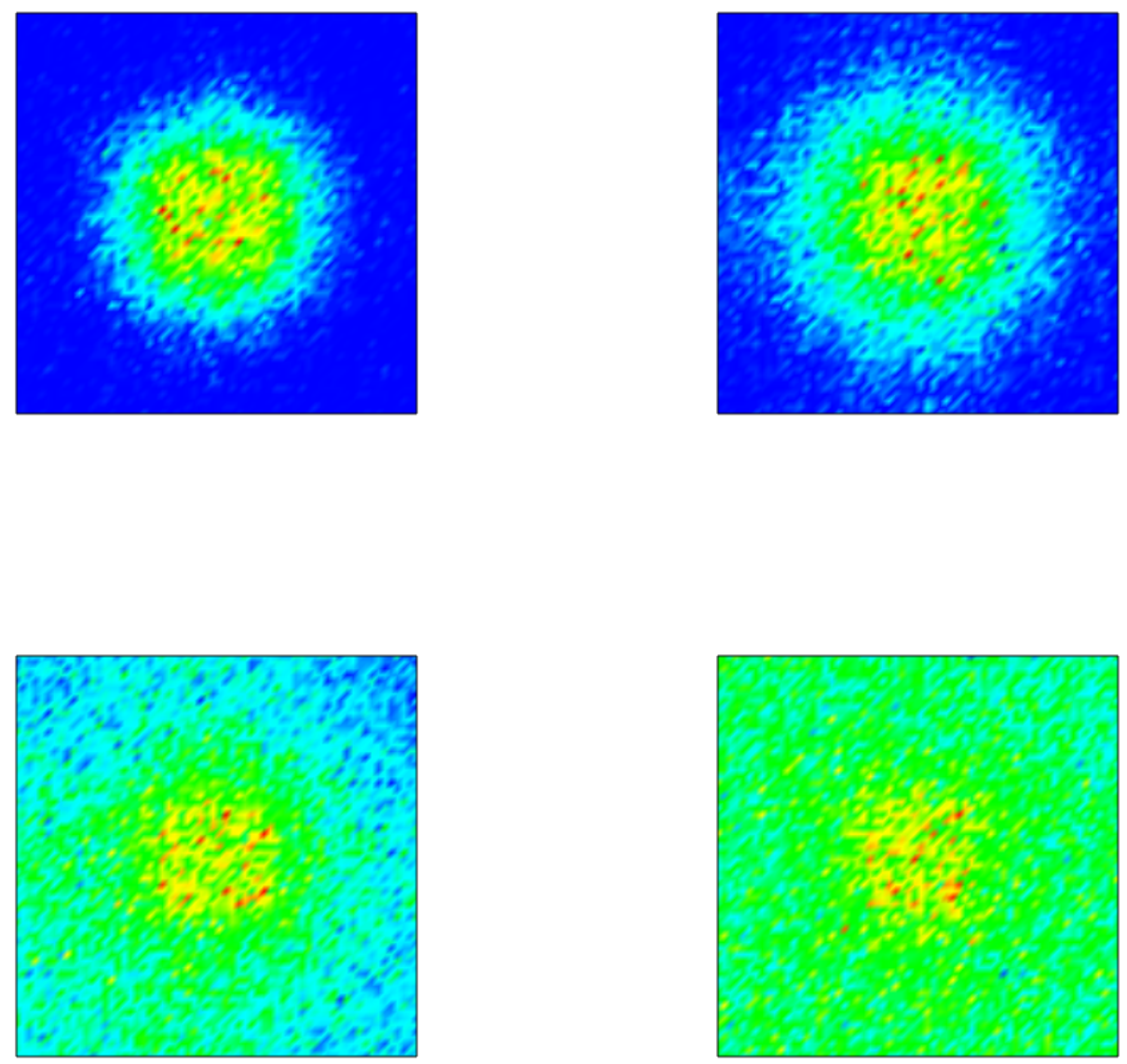

Figure 4.9: Snapshots showing the propagation of receptor phosphorylation when averaged over an ensemble of 25 runs. The pictures show a membrane patch of size $4 \times 4 \mu \mathrm{m}^{2}$ at times $t=2 \mathrm{~s}$ (upper left), $t=6 \mathrm{~s}$ (upper right), $t=10 \mathrm{~s}$ (lower left), and $t=14 \mathrm{~s}$ (lower right) after stimulation. A colour coding scheme is used for depicting the amount of phosphorylated receptors within the membrane, i.e. blue regions indicate the absence of phosphorylated receptors whereas the colors from green to red account for an increasing presence of these molecules in the respective part of the membrane. 
Figure 4.9 in which we use a colour scheme to code for the number of phosphorylated receptors in the membrane. Blue accounts for the absence of phosphorylated receptors whereas green, yellow and red indicate an increasing concentration of these molecules in the respective part of the membrane. Obviously phosphorylation is spread by a propagating pulse, which proves our overall claim that the three dimensional model is very well capable of capturing the experimental findings.

\subsection{Molecular crowding}

Up to now we have implicitly assumed that signalling occurs in a homogeneous medium, in which the molecules could freely diffuse to any place within the volume. However, this assumption is certainly not true when dealing with biological systems, which exhibit a high content of macromolecules. For this reason biological reaction media such as the cytosol or the cell membrane are said to be "crowded". This means that a significant fraction of the total volume is occupied by molecules and macromolecular assemblies other than the chemical species under investigation [Ellis, 2001]. Overall this has a variety of different effects on the chemistry which occurs inside of cells. First of all the medium is obstructed by the high macromolecular content so that diffusion is restricted. Consequently, diffusion is anomalous over short distances meaning that the mean space displacement of a Brownian walker $\left\langle r^{2}\right\rangle$ scales to time with a power smaller than one [Saxton, 1994]. A similar effect can also be observed in the reaction kinetics which become fractal. This means that the order of a reaction is no longer given by an integer value but by a rational number instead [Kopelman, 1988]. Overall we therefore expect that the dynamics of biochemical reaction networks in real cellular environments differ substantially from dynamics of the same signalling cascades studied in an idealised test tube world.

In the following we will therefore investigate how an inhomogeneous medium affects long range signal propagation. When doing so, we will first focus on the example of the EGF receptor system before we turn to the more general question of how the propagation of a chemical wave front in a stochastic reaction diffusion system is affected by inhomogeneities in the reaction medium.

\subsubsection{Lateral signal propagation in the presence of molecular crowding}

A common approach for modelling chemical signalling networks in inhomogeneous media is by obstructing the reaction medium with rigid blocks [Lipkow et al., 2005]. However, when doing so, it is by no means obvious how large these obstacles have to be and in which abundance they occur in the reaction medium. In what follows, we therefore first calibrate our model with the help of the work reported in [Tischer, 2005] before we 
actually turn to modelling the signalling cascade responsible for the spreading of EGF receptor phosphorylation.

In [Tischer, 2005] the author developed a technique called Thermal Noise Imaging Microscopy (TNIM), which allows for the study of nanoscale diffusion of a particle in a heterogeneous medium. This is accomplished by combining an optical trap with a standard light microscope such that the diffusive motion of a nanoparticle can be monitored while it is confined to a small subvolume. Obviously the trajectory of the diffusing particle also encodes information about the size and shape of the inhomogeneities in the vicinity of that particle. In the latter part of his thesis, the author used TNIM to study the diffusive motion of EGF receptors tagged with GFP in realistic biological membranes. First of all he could show that the diffusive motion of these molecules is indeed restricted. Moreover, he found that the width of typical obstacles lies in a range between $50 \mathrm{~nm}$ and more than $100 \mathrm{~nm}$. Finally he could also measure the nanoscale diffusion coefficient of the EGF receptors which has a value $D_{\text {nano }}=2.8 \mu \mathrm{m}^{2} \mathrm{~s}^{-1}$. Notice, that this value is in good agreement with typical values reported for the diffusion coefficients of proteins embedded in homogeneous membranes. However, when measuring the macroscopic diffusion coefficient of a receptor molecule embedded in the plasma membrane of a cell by Fluorescent Recovery After Photobleaching (FRAP), one normally obtains values that are 10 to 100 times lower, i.e. $D_{F R A P}=0.015-0.2 \mu \mathrm{m}^{2} \mathrm{~s}^{-1}$. This implies that if we measure the mean square displacement $\left\langle r^{2}\right\rangle$ of a particle diffusing with a diffusion coefficient $D_{\text {nano }}$ on an inhomogeneous membrane with a size equivalent to the size which is typically bleached in a FRAP experiment, then we should get the same dependence between $\left\langle r^{2}\right\rangle$ and $t$ as if the particle diffused in a homogeneous medium with a diffusion coefficient of $D_{F R A P}$.

With the above argumentation we now have a method at hand with which we can determine the density of obstacles in the membrane. According to our reasoning the physiological correct obstacle density is the one for which the average mean square displacement is 10 to 100 times slower than the average mean square displacement measured on a homogeneous grid. Consequently we simulate the random walk of a Brownian particle on a grid of the size of $4 \mu \mathrm{m} \times 4 \mu \mathrm{m}$ in which a varying proportion of the gridcells is occluded by obstacles. The obstacles are randomly distributed and each obstacle exactly covers one subvolume. Since the edge length of a gridcell is equal to $62.5 \mathrm{~nm}$, the size of the obstacles used in our approach is in accordance with the values reported in [Tischer, 2005]. The results for three different obstacle densities are displayed in the left plot of Figure 4.10, in which we have depicted the mean square displacement of a Brownian walker diffusing on grids with obstacle densities $0.0,0.2$, and 0.4 . The diffusion coefficient was set to $2 \mu \mathrm{m}^{2} \mathrm{~s}^{-1}$. The average mean square displacement measured on the homogeneous grid roughly corresponds to the theoretical prediction $\left\langle r^{2}\right\rangle=4 D t$. As opposed to that the trajectories obtained for obstacle densities 0.2 and 0.4 show a pronounced derivation from this behaviour. In fact we see that diffusion has become anomalous, i.e. we can 

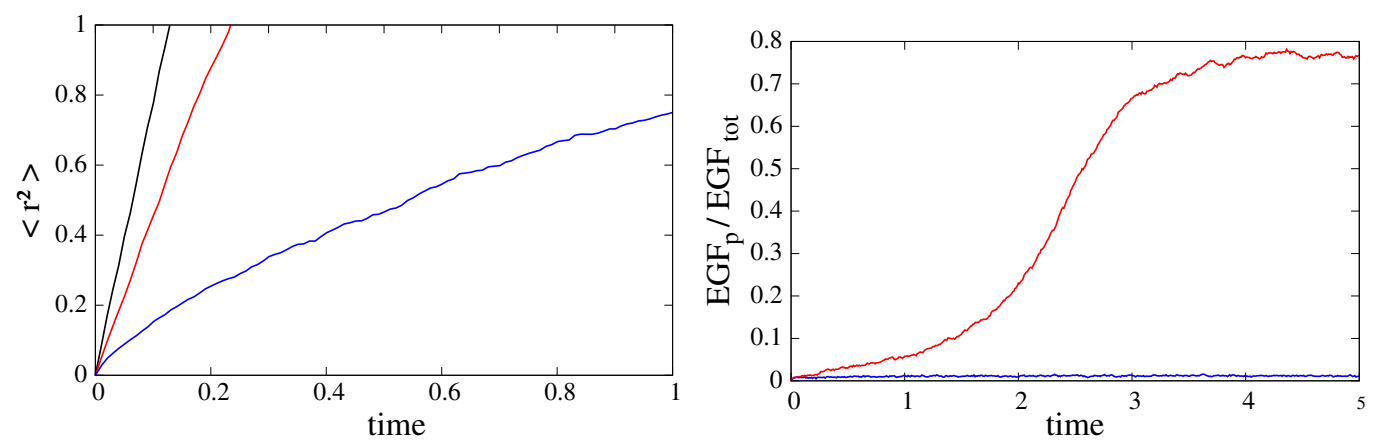

Figure 4.10: Left: Diffusion in the presence of obstacles. Right: Simulation results showing that the behaviour of the system can be changed by molecular crowding. The system resides in the uninduced state if the medium is homogeneous (blue curve). As opposed to that, it is driven into the persistently active state when obstructing $40 \%$ of the medium by obstacles

no longer fit a straight line to the data. Obviously, this shows that the average mean square displacement scales to time with a power other than one. One way to describe this would be by introducing length-scale dependent anomalous diffusion coefficients [Saxton, 1994]. More importantly we also see that the Brownian walker, which diffuses on a grid containing $40 \%$ of obstacles is more than ten times slower when covering an average mean square distance of $1 \mu \mathrm{m}^{2}$, which is a typical value for the size of the area bleached in a FRAP experiment. Moreover, it seems as if it is not possible to lower the mean square displacement by a factor of 100 . However, so far all obstacles were chosen to be of the same size and they were distributed isotropically. Both assumption do not apply for real systems. In an additional simulation we therefore introduced correlations among the obstacles and varied their sizes. The results clearly show that one can decrease the average mean square displacement in this case even further. Nevertheless, we conclude that covering $40 \%$ of the gridcells with obstacles is a good start for modelling a biomembrane with inhomogeneities.

After having seen that a value of 0.4 is a physiologically realistic estimation for the obstacle density in the membrane, we will now use this value for simulating both the two as well as the three dimensional EGF receptor system in the presence of obstacles. We use the same dimensionless parameters and the same values for the total receptor and phosphatase concentrations as before. Namely we set $\alpha_{2} / \alpha_{1}=10, I / R=100$, and $P / K=250$. In particular we are interested in whether the two systems are still bistable in the presence of inhomogeneities. When simulating the two dimensional system with inhomogeneities nothing much changes. The system is still bistable and it either settles to the uninduced or to the persistently active state. However, the mean values which we obtain for the fraction of $E G F_{p} / E G F_{\text {tot }}$ in the uninduced and in the persistently active 
state differ from the values obtained on a homogeneous medium. In particular the value for the uninduced state is larger than the value obtained on a homogeneous membrane, whereas the persistently active state takes on a lower value. Consequently we expect that the system with obstacles will already show spontaneous stochastic switching between the states for parameters where the homogeneous system would still be perfectly bistable. For the three-dimensional system, the effects of the obstacles on the behaviour of the signalling cascade are more dramatic. Here the simulation reveals that bistability can be lost. This is visualised in the right part of Figure 4.10, in which we have depicted the result of the three dimensional simulation. When simulating the system in an homogeneous medium starting with only having active phosphatases and unphosphorylated receptors, the system stays in the uninduced state (blue trajectory). As opposed to that, the system always settles to the persistently active state no matter with which initial condition we start, if we occlude the reaction medium (red trajectories). This behaviour is however well understandable, if we remind ourselves that the rate $R_{3 D}$ for reactivating the phosphatases is proportional to their diffusion coefficient; i.e. we had $R_{3 D}=\sqrt{K R D_{v}}=\sqrt{D_{p}, k_{4}}$. Moreover we have just seen that diffusion slows down if the reaction medium contains obstacles. Consequently, $R_{3 D}$ is decreased. In physical terms, this simply means that the exchange of inactive phosphatase molecules at the membrane with inactive phosphatase molecules from the bulk is hampered by obstacles such that the system is driven into the persistently active state. Furthermore, the correctness of our argumentation can be seen when simulating the system with a higher diffusion coefficient which makes up for the hampering of the obstacles. In this case bistability is recovered.

\subsubsection{Chemical wave fronts in an inhomogeneous medium}

After having analysed the steady state behaviour of the EGF receptor system in an inhomogeneous medium we will now turn to the more general question of whether chemical wave fronts provide a reliable means for propagating a signal over long distances if the reaction medium is occluded by obstacles. In particular, we investigate whether a front remains stable and continues to propagate with velocities allowing for long-range signalling. However, in order to reduce the complexity of the problem we will change the reaction system under investigation. Instead of simulating the EGF receptor system, we will turn to a prototypical system capable of exhibiting propagating fronts. In particular, we will continue our analysis by simulating a simple autocatalytic reaction system with only one chemical species $A$ and the reversible reaction

$$
A \underset{r}{\stackrel{k}{\rightleftharpoons}} A+A
$$

With $u_{A}=n_{A} / \Omega$ accounting for the concentration of the molecular species $A$ in the reaction volume $\Omega$, the above reaction scheme gives rise to the following reaction diffusion 
equation:

$$
\partial_{t} u=D \triangle u+k u-r u^{2} .
$$

This equation possesses two steady states $\bar{u}_{u}=0$ and $\bar{u}_{s}=k / r$ with the former being the unstable and the latter being the stable steady state. Hence, we arrive at the rescaled equation

$$
\partial_{t} u=\triangle u+u(1-u),
$$

when introducing the substitutions $u^{*}=u / \bar{u}_{s}, t^{*}=k t, x^{*}=x(k / D)^{1 / 2}$ and omitting the asterisks for notational convenience. This equation, which is generally referred to as the Fisher equation, has been extensively studied [Murray, 1993]. In one spatial dimension, equation (4.21) gives rise to a number of front solutions $u(x-v t)$ with the velocity $v$ satisfying the condition $v \geq v_{\min }=2 \sqrt{k D}$.

In the following we will now use our Monte Carlo simulation environment in order to investigate whether the above system admits for stable wave fronts in a two dimensional inhomogeneous medium. For modelling inhomogeneities we use the same approach as described above, i.e. we introduce rigid obstacles which cover exactly one reaction cell. The fraction of grid cells covered by obstacles is chosen out of a range from 0 to 0.4 . If the density of covered grid cells is increased even further, the proportion of free grid cells drops below the percolation threshold. Hence, a system spanning cluster of free cells does no longer exist in an infinite system so that the fronts get stuck in the simulated finite system. At time $t=0$ the particle density is set to the stable steady state value in all nonblocked gridcells attached to the left boundary of our rectangular reaction medium. Such an initial condition leads to an interface line

$$
x_{I}(y)=\max _{x}\{u(x, y)>0.5\}
$$

separating the regions of high and low particle concentration. This line moves into the positive $x$ direction. One can easily extract the sigmoidally shaped profile by projecting the particle density onto the $x$ axis. For monitoring the displacement of the propagating wave, we define the mean interface position as

$$
\alpha=\frac{l}{L} \sum_{y} x_{I}(y),
$$

with $L$ being the length of the grid in $y$ direction and $l$ accounting for the length of a subvolume. Furthermore we define the roughness of the interface as the deviation of the interface from its mean position $\alpha$, i.e.

$$
\triangle^{2}=\frac{l}{L} \sum_{y}\left(x_{I}(y)-\alpha\right)^{2} .
$$



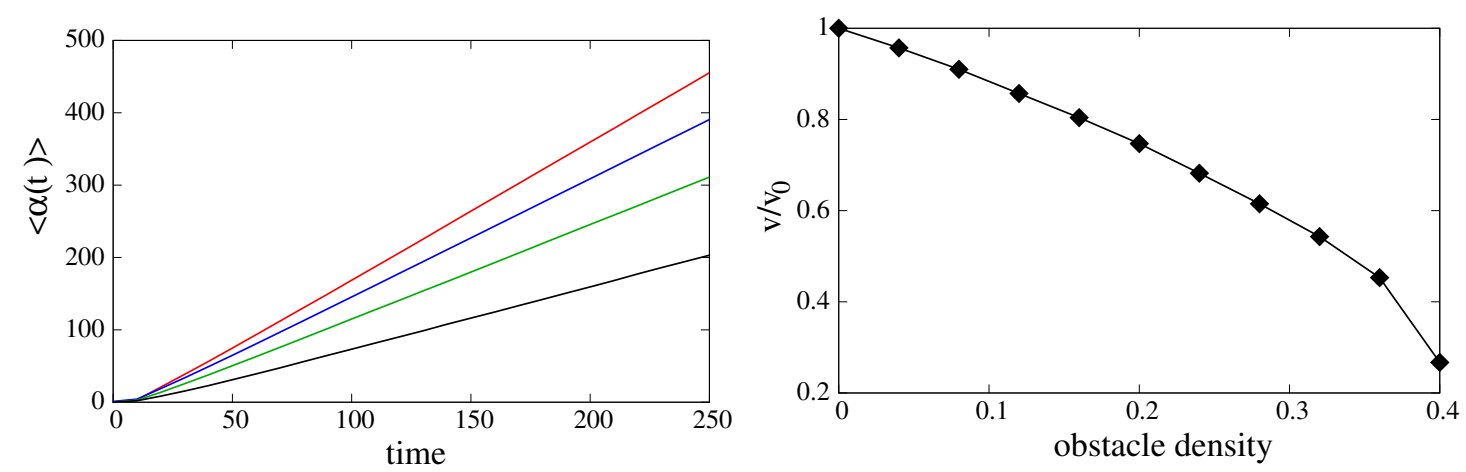

Figure 4.11: Left: Mean position of the interface as a function of time. The different colours account for the different obstacle densities: red corresponds to 0 , blue to 0.12 , green to 0.24 , and black to 0.36 . Right: Normalised velocity as a function of the obstacle density.

First we analyse how the propagation of the mean interface position is affected by inhomogeneities. As can be seen in Figure 4.11, in which we have depicted the mean interface position as a function of time, the average wave front always propagates with a constant velocity through the reaction medium, no matter by how many obstacles the medium is occluded. Unlike simple diffusion the scaling behaviour of the displacement of the propagating front is not changed by an inhomogeneous medium. However, the velocity with which the front propagates through the reaction medium clearly decreases when including obstacles. This can be seen in right part of Figure 4.11 in which the average velocity normalised to the homogeneous velocity is shown as a function of the obstacle density. For low obstacle densities we first see a linear decrease in the velocity. As we approach the percolation threshold, the velocity becomes increasingly slower, since macroscopic obstacle cluster begin to appear in the medium. So far, we have only analysed the ensemble average. However, when taking a look at individual trajectories one observes a somewhat different behaviour (Figure 4.12). Especially at high obstacle densities one finds, that the motion of the waves is composed of rapid jumps alternating with periods during which the interface advances very slowly. This behaviour is an effect of the underlying obstacle distribution. In particular an interface slows down, whenever it has to surmount a macroscopic obstacle cluster. Consequently we observe an unsteady propagation of the mean position of the interface.

Next we will investigate how the roughness of the interface is changed, if the reaction medium contains inhomogeneities. This part of the study is motivated by the work of [Riordan et al., 1995], who showed that the interface of a chemical wave front broadens during its propagation, if one accounts for the noise in the underlying system. However, the simulations in that study were performed on a homogeneous medium. Hence, we now want to examine how the finding of [Riordan et al., 1995] has to be altered when accounting for obstacles in the reaction medium. Overall, our simulations give rise to the 

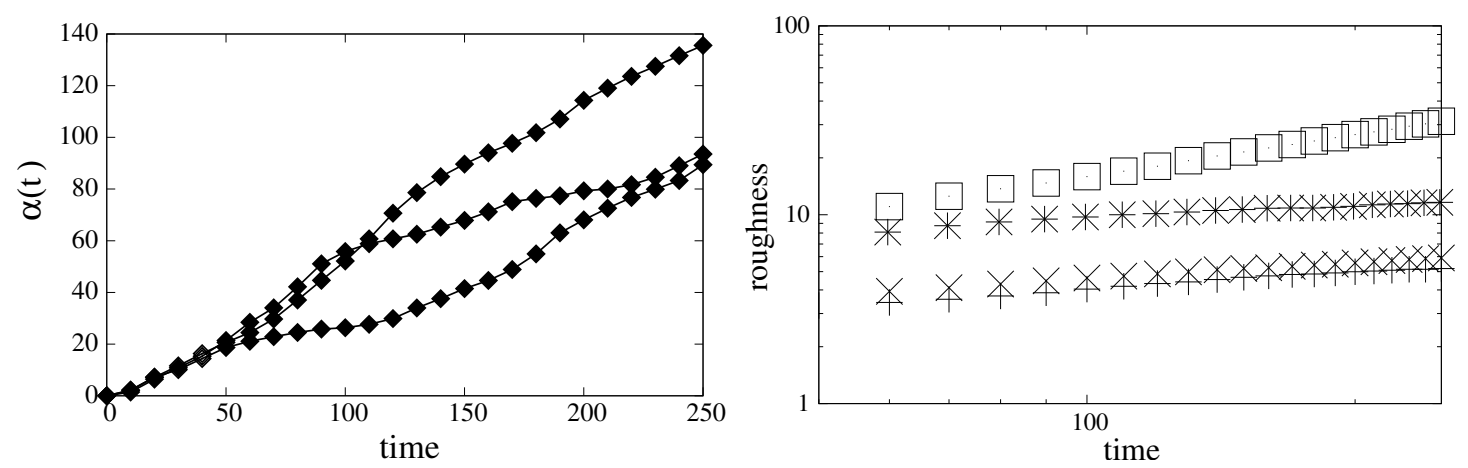

Figure 4.12: Left: Three sample trajectories of the mean interface position as a function of time obtained when simulating the system with a value of 0.4 for the obstacle density. Right: Average roughness as a function of time. The trajectories correspond to obstacle densities $0.0,0.24,0.36$, and 0.4 (from bottom to top).

results depicted in the right part of Figure 4.12. In this figure, we have used a double logarithmic scale to plot the roughness of the front as a function of time. The trajectories were obtained by simulating the system on a homogeneous grid and on grids with obstacle densities $0.24,0.36$, and 0.4 (from bottom to top). When analysing the results one finds that the roughness of the wave front is not affected when only a few obstacles occlude the reaction medium. In fact the trajectory obtained on a grid with an obstacle density of 0.24 almost perfectly matches with the result obtaine from a homogeneous medium. If we now increase the obstacle density further, the interface becomes rougher (obstacle density 0.36 ). Finally if the obstacle density gets close to the percolation threshold, we observe that the interface grows a lot faster than on a homogeneous medium. In [Riordan et al., 1995] the authors report that the roughness of a front on a homogeneous medium scales asymptotically in time with an exponent equal to 0.272. Although we have not reached the asymptotic regime we find a similar scaling exponent $(0.265)$. Interestingly, if we fit power laws to the results obtained for obstacle densities $0.24,0.36$ and 0.4 , we obtain values of $0.263,0.18$ and 0.736 respectively. Overall these results imply that we have to distinguish between three different obstacle regimes when analysing the roughness of the front. If there are no or relatively few obstacles embedded in the reaction medium, the interface disperses as shown by [Riordan et al., 1995]. For high obstacles densities close to the percolation threshold the interface quickly disintegrates because macroscopic clusters appear in the reaction medium. However, for intermediate obstacle densities it seems as if inhomogeneities cause a focusing of the interface as it does not disperse as quickly as on a homogeneous medium. However, we have to point out that one has to be very careful when interpreting these results because the values were not obtained in the asymptotic regime. Obviously, the correspondence of the value obtained on a homogeneous grid with the asymptotic value does not imply that all the other scaling exponents also correspond to the real (i.e. asymptotic) values. One would have to 
run longer simulations in order to obtain reliable results. Furthermore, from a biological perspective the asymptotic behaviour will be of minor importance, because biological systems simply do not live long enough to ever reach the asymptotic regime.

So far the obstacles were always distributed isotropically meaning that each obstacle covered exactly one subvolume. In the final set of simulations, we change this and investigate how linear obstacles influence the front's speed and roughness. Within all the simulation a fixed proportion of $32 \%$ of the reaction cells were covered by obstacles. However, a single obstacle now covered up to four grid cells and all obstacles were oriented either horizontally or vertically, i.e. either in the direction of front propagation or perpendicular to it. Our simulation results showed that vertically oriented obstacles slow the propagation down whereas the horizontally distributed obstacles speed up propagation as compared to the isotropic case. At the same time the roughness of the front is reduced for vertically distributed and increased for horizontally distributed obstacles. A hand-waving explanation of this behaviour may easily be given. With increasing obstacles sizes the number of obstacles heads or tails pointing in the direction of the motion of the wave front decreased, if the obstacles are oriented horizontally. Thus there will be less effective obstacles hampering the front's motion. At the same time the roughness of the front increases because balancing the front becomes increasingly different. For vertically oriented obstacles the opposite argumentation applies. Balancing the front is facilitated because the motion in direction of the propagation is hampered. Overall, these last results therefore show that it is not only the density of the obstacles which matters but that also their size, shape, and orientation are of great significance.

When setting our results in relation to the initial question of whether chemical wave fronts provide a reliable mechanism for long range chemical signalling, the answer is ambiguous. On the one hand we have seen that the chemical wave front propagates with constant velocity for all admissible obstacle values. Viewed from this perspective, chemical wave fronts therefore seem to provide a suitable mechanism for long range chemical signalling. Eventually a signal will always arrive at the destination. However, we have also seen that the roughness of the interface increases as the wave propagates through the medium. In particular the interface disperses even in a homogeneous medium and in a medium containing obstacles the dispersion of the interface becomes even larger. Taken together with the finding of the previous subsection that realistic values for the obstacle density lie close to the percolation threshold, it becomes questionable whether chemical wave fronts provide a reliable mechanism for propagating a signal over long distances because the arrival time of the signal at a specific point on the membrane can not be predicted any more. 
76 CHAPTER 4. VARIATION 2: MONTE CARLO SIMULATION OF LATERAL SIGNAL PROPAGATION 


\section{Variation 3: Representing space revisited}

in which we present the Transformed Grid Monte Carlo and the Finite Volume Monte Carlo method with which one can represent the geometry of a reaction medium in a more realistic way than with the approach presented in the second chapter. In the latter part of this chapter we use the Transformed Grid Monte Carlo approach for simulating a minimal model of the gradient sensing signalling cascade in Dictyostelium discoideum. Our model adapts perfectly to uniform stimuli and functions as a switch if the system is exposed to linear gradients.

5.1 Aim and Background . . . . . . . . . . . . . 77

5.2 Transformed Grid Monte Carlo . . . . . . . . . . . . 79

5.2.1 Cylindrical coordinates . . . . . . . . . . . . . 79

5.2 .2 Spherical coordinates . . . . . . . . . . . . 80

5.2.3 Combing different geometries for the modelling of realistic biological structures . . . . . . . . . . . . . 81

5.2 .4 General case . . . . . . . . . . . . . . . . 85

5.3 Finite Volume Monte Carlo . . . . . . . . . . . . 90

5.4 Eukaryotic Chemotaxis . . . . . . . . . . . . 93

5.4.1 Gradient Sensing . . . . . . . . . . . . . . . . . 93

5.4.2 Computational Modelling of Gradient Sensing . . . . . 95

5.5 Discussion . . . . . . . . . . . . 100

\subsection{Aim and Background}

In the second chapter we have set up a stochastic theory of spatially inhomogeneous reaction systems using a simple type of well-stirred subvolumes, i.e. small cubes, which form a regular cubic lattice. However, it is obvious that real geometries in which chemical signalling takes place are not adequately described by cubes. Hence, if one nevertheless approximates real geometries by a set of cubes, one either obtains a crude spatial representation or an inefficient simulation if the size of the cubes is chosen too small. Furthermore, one wants to have a simulation environment in which the size of the grid- 

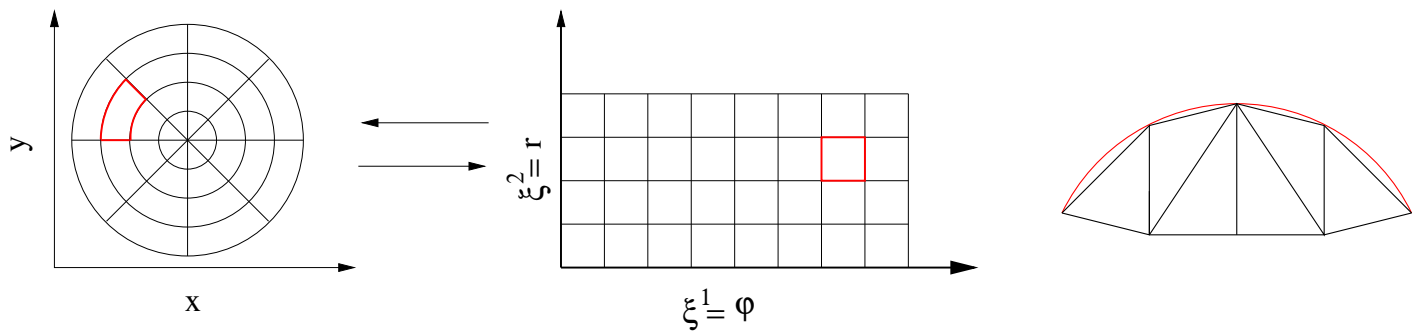

Figure 5.1: Left: General idea of the TGMC method; a cubic lattice is mapped onto polar coordinates. Right: General idea of the FVMC method; the reaction medium is simplicial cells.

cells can be varied. That way one can choose a fine spatial discretisation for those parts of the reaction medium in which the probability of the occurrence of reactions is high. As opposed to that one would choose a crude spatial representation if this probability is low. In fact we have employed this strategy in the fourth chapter for the simulation of the three dimensional EGF receptor system.

In the following, we will therefore generalise our Monte Carlo method to coupled subvolumes of more general geometrical shapes and will take care of a discretisation, which conforms as well as possible to the physical boundaries of the system. We will put forward two strategies, which correspond to two standard approaches of grid generation in numerical problems and computer geometry (see for instance [Knabner and Angermann, 2000] and [Hoschek and Lasser, 1992]). The first strategy, which is visualised in the left part of Figure 5.1 transforms a regular lattice grid such that the system boundary becomes a coordinate surface (or line) in the transformed coordinates. This approach is called grid transformation. The second approach divides the system's interior into simplicial cells such that boundary points correspond to corners of these simplices. The idea of this approach is visualised in the right part of Figure 5.1. Simplicial discretisations are common in finite element and finite volume methods used for the numerical solution of (deterministic) partial differential equations. In combination with our Monte Carlo method we will call the resulting algorithms Transformed Grid Monte Carlo (TGMC) and Finite Volume Monte Carlo (FVMC), respectively.

We adopt the notation introduced in the second chapter and divide the system volume $\Omega$ into well-stirred subvolumes $\Omega_{k}$, which are represented by a conveniently chosen point $\boldsymbol{r}_{k}$ of its interior. In the present section, however, the volumes $\left|\Omega_{k}\right|$ and geometrical shapes may vary from subvolume to subvolume. Our task is to construct a hopping Hamiltonian

$$
\hat{H}^{h o p}=-\sum_{i=1}^{M} \sum_{k} \sum_{\mu=1}^{N(k)} K_{i, k, a}\left[\hat{\eta}_{i}\left(\boldsymbol{r}_{k}+\boldsymbol{b}_{a}\left(\boldsymbol{r}_{k}\right)\right)-\hat{\eta}_{i}\left(\boldsymbol{r}_{k}\right)\right] \hat{\varphi}_{i}\left(\boldsymbol{r}_{k}\right)
$$

which corresponds to diffusive motion on length-scales, which are large compared to 
the subvolume sizes. Equ. (5.1) generalises equ. (2.22). Notice, that the number of neighbours $N(k)$ may be different for different subvolumes and may include more than just nearest neighbours. Finally the vectors $\boldsymbol{b}_{a}\left(\boldsymbol{r}_{k}\right)$, which connect $\boldsymbol{r}_{k}$ with a neighbour may also depend upon $k$. The strategy to construct the hopping rates $K_{i, k, a}$ will be the same as in section 2.3 in that we compare the discretized version of $\int d^{3} \boldsymbol{r} \varphi \nabla^{2} \eta$ with the hopping Hamiltonian equation(5.1).

\subsection{Transformed Grid Monte Carlo}

We begin by fixing our notation. Let $\boldsymbol{e}_{i}, i=1,2,3$ be a Cartesian vector basis in the three dimensional physical space. Vectors in this space are denoted by

$$
\boldsymbol{r}=x^{i} \boldsymbol{e}_{i}
$$

Here and in the following, we make use of Einstein's summation convention, meaning that a summation over each index is applied, if it appears both raised and lowered in an expression. A point in space can be characterised by the three components $x^{i}$ and the coordinate lines $\mathcal{L}^{i}=\left\{\boldsymbol{r}\left(x^{i}\right) \mid x^{k}=\right.$ const for $\left.k \neq i\right\}$ are straight and mutually orthogonal. The grid lines, which we use for calculational purposes will now be chosen as coordinate lines of a new curvilinear non-orthogonal coordinate system, which in general will be non-orthogonal. This coordinate system is parametrised by coordinates $\xi^{\mu}, \mu=$ $1,2,3$ and grid lines correspond to $\mathcal{L}^{\mu}=\left\{\boldsymbol{r}\left(\xi^{\mu}\right) \mid \xi^{\nu}=\right.$ const for $\left.\nu \neq q \mu\right\}$. In particular, we require that the system boundary corresponds to a coordinate surface, i.e. $\partial \Omega=$ $\left\{\boldsymbol{r}\left(\xi^{1}, \xi^{2}, \xi^{3}\right) \mid \xi^{3}=\right.$ const $\}$. Furthermore, it should be noted here, that we always assume the mapping to be sufficiently smooth (at least two times continuously differentiable). On the other hand, our method will not require the map to be given in analytical form, so that grids emerging from experimentally determined boundaries may be used. All necessary operations will ultimately be performed numerically.

Nevertheless, important simple cases are instructive and therefore we will first give explicit formulae for cylindrical and for spherical coordinate systems before we embark to the general case of a curvilinear non-orthogonal coordinate system. For these two simple cases, we will give explicit expressions for the map. Furthermore, we will show that the simple examples can be combined such that one obtains more complex shapes suitable for simulating chemical signalling.

\subsubsection{Cylindrical coordinates}

In cylindrical coordinates the $\xi^{\mu}$ correspond to $r, \phi$ and $z$ and

$$
\begin{aligned}
& x^{1}=r \cos \phi \\
& x^{2}=r \sin \phi
\end{aligned}
$$




$$
x^{3}=z
$$

We start from a grid in $(r, \phi, z)$-space with grid points $r_{k}, \phi_{l}, z_{m}$ (k,l,m integer) and grid spacings $d r, d \phi, d z$. The Laplace operator, which has to be discretized, takes on the form

$$
\Delta=\partial_{r}^{2}+\frac{1}{r} \partial_{r}+\frac{1}{r^{2}} \partial_{\phi}^{2}+\partial_{z}^{2}=\Delta_{r}+\Delta_{\phi}+\Delta_{z} .
$$

A finite difference discretisation on the $r, \phi, z$ grid with lattice is readily obtained up to second order accuracy. $\Delta_{r}$ may be written in the form

$$
\left.\Delta_{r} \eta\right|_{k, l, m}=\frac{1}{d r^{2}}\left[\left(1+\frac{d r}{2 r_{k}}\right)\left(\eta_{k+1}-\eta_{k}\right)+\left(1-\frac{d r}{2 r_{k}}\right)\left(\eta_{k-1}-\eta_{k}\right)\right]+O\left(d r^{2}\right)
$$

with $\eta_{k}$ stands for $\eta\left(r_{k}, \phi_{l}, z_{m}\right)$ so that $\eta_{k \pm 1}=\eta\left(r_{k} \pm \Delta_{r}, \phi_{l}, z_{m}\right)$. Comparing this expression with equation.(5.1), we can simply read off the hopping rates between neighbouring subvolumes in radial direction.

$$
K_{i, k \rightarrow k \pm d r}=\frac{D_{i}}{d r^{2}}\left(1 \pm \frac{d r}{2 r_{k}}\right)
$$

The other rates are calculated analogously such that one obtains the rates summarised in Table 5.1, which is given below.

\begin{tabular}{lr}
\hline Direction & Hopping rate \\
\hline$r_{k} \rightarrow r_{k} \pm d r$ & $D_{i}\left(1 \pm d r / 2 r_{k}\right) / d r^{2}$ \\
$\phi_{l} \rightarrow \phi_{l} \pm d \phi$ & $D_{i} /\left(d \phi^{2} r_{k}^{2}\right)$ \\
$z_{m} \rightarrow z_{m} \pm d z$ & $D_{i} / d z^{2}$ \\
\hline
\end{tabular}

Table 5.1: Hopping rates that have to be employed when simulating diffusion in cylindrical coordinates.

\subsubsection{Spherical coordinates}

In spherical coordinates the $\xi^{\mu}$ correspond to the coordinates $r, \theta, \phi$ and

$$
\begin{gathered}
x^{1}=r \sin \theta \cos \phi \\
x^{2}=r \sin \theta \sin \phi \\
x^{3}=r \cos \theta .
\end{gathered}
$$


We start from a grid in $(r, \theta, \phi)$-space with grid points $r_{k}, \theta_{l}, \phi_{m}(k, l, m$ integer) and grid spacings $d r, d \theta, d \phi$. The Laplace operator $\Delta=\Delta_{r}+\Delta_{\theta}+\Delta_{\phi}$ takes on the form

$$
\begin{gathered}
\Delta_{r} \eta=\frac{1}{r^{2}} \partial_{r}^{2} \eta+\frac{2}{r} \partial_{r} \eta \\
\Delta_{\theta}=\frac{1}{r^{2}} \partial_{\theta}^{2} \eta+\frac{\cot \theta}{r^{2}} \partial_{\theta} \eta \\
\Delta_{\phi}=\frac{1}{r^{2} \sin ^{2} \theta} \partial_{\phi}^{2} \eta
\end{gathered}
$$

and the discretisations look as follows:

$$
\begin{gathered}
\left.\Delta_{r} \eta\right|_{k, l, m}=\frac{1}{d r^{2}}\left[\left(1+\frac{d r}{r_{k}}\right)\left(\eta_{k+1}-\eta_{k}\right)+\left(1-\frac{d r}{r_{k}}\right)\left(\eta_{k-1}-\eta_{k}\right)\right]+O\left(d r^{2}\right) \\
\left.\Delta_{\theta} \eta\right|_{k, l, m}=\frac{1}{r_{k}^{2} d \theta^{2}}\left[\left(1+\frac{d \theta \cot \theta_{l}}{2 r_{k}^{2}}\right)\left(\eta_{l+1}-\eta_{l}\right)+\left(1-\frac{d \theta \cot \theta_{l}}{2 r_{k}^{2}}\right)\left(\eta_{l-1}-\eta_{l}\right)\right] \\
\left.\Delta_{\phi} \eta\right|_{k, l, m}=\frac{1}{d \phi^{2} \sin ^{2} \theta_{l}}\left[\eta_{m+1}-\eta_{m}+\eta_{m-1}-\eta_{m}\right] .
\end{gathered}
$$

Again by comparison with equation (5.1) one obtains the hopping rates which are listed in the table below.

\begin{tabular}{lr}
\hline Direction & Hopping rate \\
\hline$r_{k} \rightarrow r_{k} \pm d r$ & $D_{i}\left(1 \pm d r / r_{k}\right) / d r^{2}$ \\
$\theta_{l} \rightarrow \theta_{l} \pm d \phi$ & $D_{i}\left(1 \pm d \theta \cot \theta_{l} / 2 r_{k}^{2}\right) / r_{k}^{2} d \theta^{2}$ \\
$p h i_{m} \rightarrow p h i_{m} \pm d \phi$ & $D_{i} / d \phi^{2} \sin ^{2} \theta_{l}$ \\
\hline
\end{tabular}

Table 5.2: Hopping rates that have to be employed when simulating diffusion in spherical coordinates.

\subsubsection{Combing different geometries for the modelling of realistic biological structures}

With the technique presented in the previous section we are now able to correctly model diffusion on meshes which are set on either Cartesian, cylindrical, or spherical coordinates. However, in order to use our approach for the simulation of chemical signalling in complex geometries, we have to combine different geometries. In the following, we will briefly show how this can be accomplished. In particular, we will present two simple examples in which set up more complex geometries. Namely we will construct a rod like bacterial cell and a dendrite with spines attached to it. 

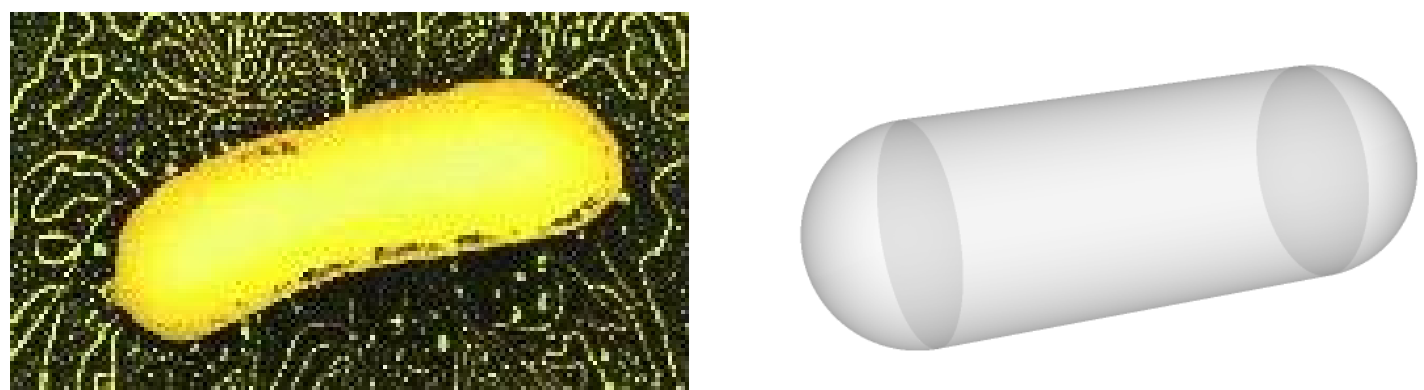

Figure 5.2: Left: Microscopic picture of a single $E$ coli cell which is loaded with a fluorescent dye. Right: Idealised geometry used for modelling single cell organisms

\section{Bacterial cell}

As a first example, we will construct a reaction medium that allows for the simulation of chemical signalling cascades in single bacterial cell such as Escherichia coli. Although bacterial cells only have a volume of approximately $1 \mu \mathrm{m}^{3}$, space nevertheless needs to be accounted for when modelling signalling cascades in these organisms. This is quite obvious if one for instance considers the Min proteins in E coli, which oscillate in order to find the middle of the cell for cell division [Fange and Elf, 2006]. But also other signalling cascades such as the cascades regulating bacterial chemotaxis depend on the shape and structure of the reaction medium [Lipkow et al., 2005]. Consequently a realistic representation of space for the modelling of chemical signalling cascades in bacterial cells is therefore highly desirable.

When taking a look at microscopic picture of E coli, such as the one on the left hand side of Figure 5.2, one immediately notices that the shape of a bacterial cell is well represented by a cigar or a rod. Hence, we can easily model such a shape by simply attaching two half spheres to a cylinder with radius $r$. Consequently we arrive at a reaction medium equal to the one visualised on the right hand side of Figure 5.2. The computational grid we obtain is similar to the one used by [Blom and Peletier, 2002]. However, unlike the grid presented there, we do not assume that the reaction medium is symmetric to the $r$-axis. Hence with our discretisation we are also able to account for variations of the molecules in the $\phi$-direction. A visualisation of the grid is given in Figure 5.3, in which the left part shows a view that is obtained when cutting the bacterium in a sagital manner and in which the right part shows a section through the transverse plane of the bacterium. In particular the cylinder and the two half spheres are discretized in 
such a way, that each cylindrical grid cell at the interface between the cap and the main body of the cell neighbours only with one spherical grid cell. The hopping rates between the two grids are determined by the continuity of the particle currents (see part on Finite Volume Monte Carlo Method).

For testing whether diffusion is correctly modelled in our reaction medium, a Brownian
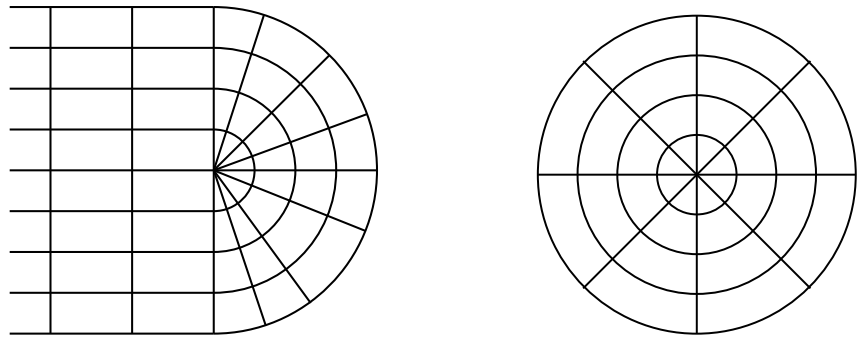

Figure 5.3: Computational grids used when modelling a single bacterial cell. The left part shows the longitudinal cut and the right part the cross section.

walker was allowed to freely diffused in the cell. As the quotient of the accumulated time which the particle spend in each gridcell $t_{i}$ and the total simulation time $T$ was equal to the quotient of the gridcell volume $\omega_{i}$ and the total volume $\Omega$, i.e. $t_{i} / T=\omega_{i} / \Omega$, we conclude that our reaction medium is well suited for modelling chemical signalling in bacterial cells.

\section{Dendrite}

After having presented a way for computationally representing the shape of single cell organisms such as Escherichia coli, we will now turn to our second example. Namely we will set up a reaction medium with which we can simulate chemical signalling in a dendrite that has spines attached to it. A possible application for our reaction medium is for instance the question of whether spines can really be regarded as isolated chemical reaction chambers or whether chemical crosstalk can occur among neighbouring spines [Ajay and Bhalla, 2006].

In the left part of Figure 5.4 we see a picture of a neuron with its dendritic tree. In the bottom part we further see a magnification of a piece of dendrite. As can be seen there, a dendrite is formed by two structures; namely by the parental dendrite and by the small protrusions, which are called spines. A spine is therefore nothing but a small membranous extrusions. Generally one further subdivides the spine into two parts. The first part is the bulbous head, which connects the dendrite with the axon of a presynaptic cell. The head also houses the signal transduction machinery of the postsynaptic side. The second part of the spine is the so-called spine neck which simply connects the main dendrite with the head. 

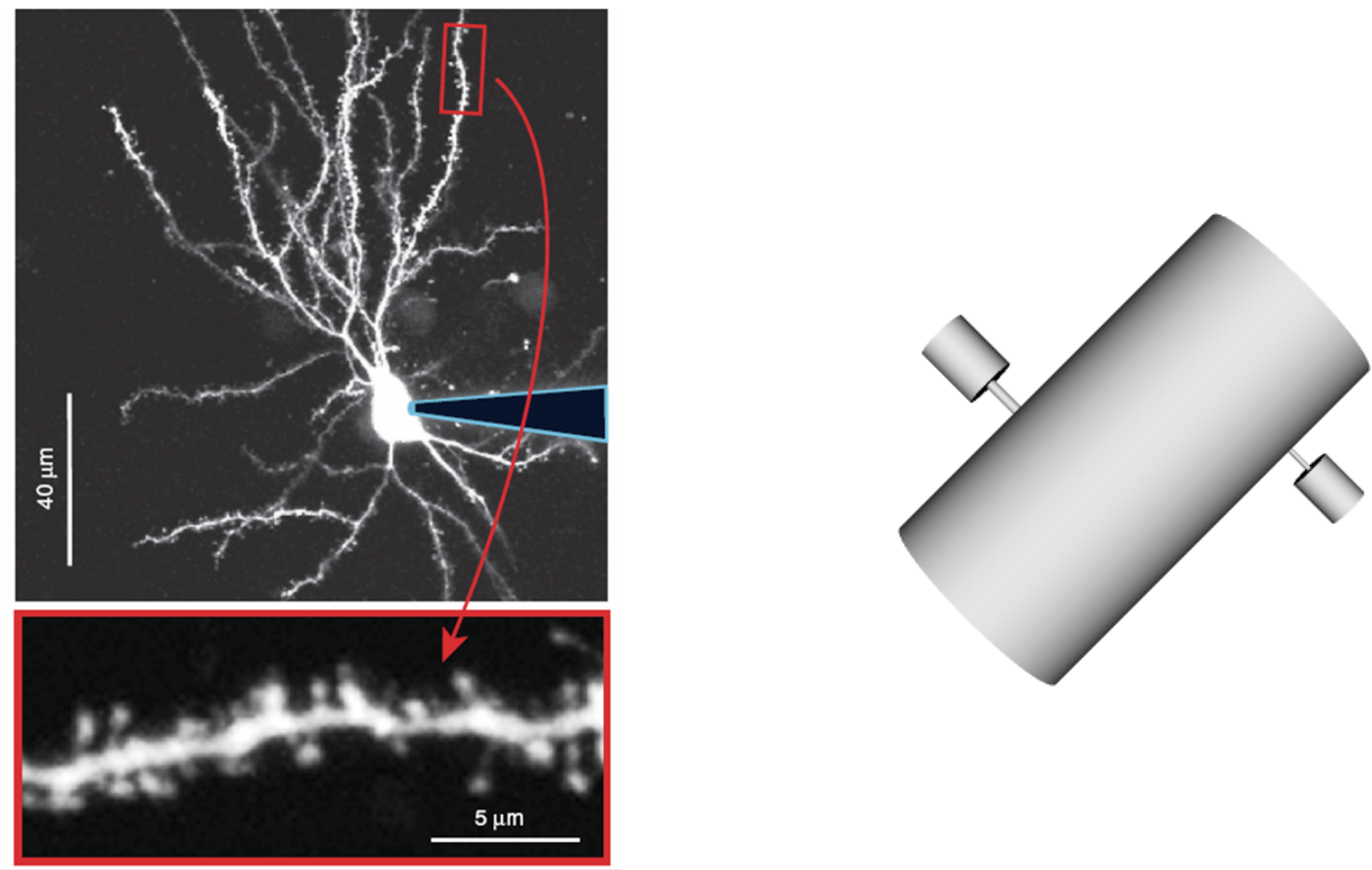

Figure 5.4: Left part: Two pictures showing a neuron and a magnified view of a piece of dendrite. Right part: A computational representation of a piece of dendrite to which spines are attached.

Generally spines come in a variety of different shapes and sizes. Basically one distinguishes between three types: thin spines, mushroom spines, and stubby spines. In a first approximation, it is however reasonable to model both the main dendrite as well as the head and neck of the different spine types by cylinders of different sizes. The main dendrite normally has a diameter of $3-4 \mu \mathrm{m}$. Compared to this the spine necks are minute as they only have a diameter of $0.1-0.2 \mu \mathrm{m}$. Typical diameters for the spine head lie around $1 \mu \mathrm{m}$ and the overall height of a spine is approximately equal to $2 \mu \mathrm{m}$. Overall, if one models a dendrite as laid out above, one obtains a reaction medium as the one visualised in the right part of Figure 5.4. The discretisation of this shape is straightforward as it only contains cylinders. The only potential problem arises at the junction between the main dendrite because one would have to account for the curvature of the main dendrite in an exact representation. However, since the difference in diameter between the spine neck and the main dendrite are so large, the spine neck effectively sits on a flat surface. Hence the interface area between these two structures is simply given by the footprint of the spine neck and the calculation of the distance between the bordering cells at the junction is also straightforward. 


\subsubsection{General case}

Let us now turn to the general case. As the $\xi^{\mu}$ coordinate system will in general be curvilinear and non-orthogonal, we have to distinguish between $\mathrm{co}^{-}$ans contravariant vectors. For convenience we first recall some standard notions of differential geometry, which will be needed for our purposes.

A local covariant vector base (3-bein) $\boldsymbol{b}_{\mu}$ is spanned by the tangent vectors at the coordinate lines, i.e.

$$
\boldsymbol{b}_{\mu}=\frac{\partial \boldsymbol{r}\left(\xi^{\mu}\right)}{\partial \xi^{\mu}}=\partial_{\mu} \boldsymbol{r}
$$

The components of the base vectors with respect to the Cartesian base are given by

$$
b_{\mu}^{i}=\partial_{\mu} x^{i},
$$

which are just the components of the Jacobian matrix of the grid transformation. The (covariant) metric tensor defined by

$$
g_{\mu \nu}=\boldsymbol{b}_{\mu} \cdot \boldsymbol{b}_{\nu}=\sum_{i=1}^{3} b_{\mu}^{i} b_{\nu}^{i}
$$

contains the information on how to compute lengths and angles from the components of vectors in the local $\boldsymbol{b}_{\mu}$ system, because for two vectors $\boldsymbol{p}=p^{\mu} \boldsymbol{b}_{\mu}$ and $\boldsymbol{q}=q^{\mu} \boldsymbol{b}_{\mu}$ the scalar product is given by

$$
\boldsymbol{p} \cdot \boldsymbol{q}=p^{\mu} q^{\nu} \boldsymbol{b}_{\mu} \cdot \boldsymbol{b}_{\nu}=p^{\mu} q^{\nu} g_{\mu \nu}
$$

An associated contravariant vector base $\boldsymbol{b}^{\mu}$ is constructed from

$$
\boldsymbol{b}^{\mu} \cdot \boldsymbol{b}_{\nu}=\delta_{\mu \nu}
$$

The corresponding contravariant metric tensor $g^{\mu \nu}$ is just the matrix inverse of $g_{\mu \nu}$. Every vector $\boldsymbol{p}$ in the vector space spanned by the tangent vectors at the $\xi$ coordinate lines can be represented either by contravariant or by covariant components

$$
\boldsymbol{p}=p^{\mu} \boldsymbol{b}_{\mu}=p^{\mu} g_{\mu \nu} \boldsymbol{b}^{\nu}=p_{\nu} \boldsymbol{b}^{\nu}
$$

For curvilinear coordinate systems the 3-bein changes in space. If we move from $r$ to $\boldsymbol{r}+d \boldsymbol{r}$, the base vectors change from $\boldsymbol{b}(\boldsymbol{r})_{\mu}$ to $\boldsymbol{b}(\boldsymbol{r}+d \boldsymbol{r})_{\mu}=\boldsymbol{b}(\boldsymbol{r})_{\mu}+d \boldsymbol{b}_{\mu}$. Let us express the infinitesimal changes

$$
d \boldsymbol{b}_{\mu}=\left(\partial_{\nu} \boldsymbol{b}_{\mu}\right) d \xi^{\nu}
$$

with respect to the 3-bein at $\boldsymbol{r}$. For this purpose one introduces the Christoffel symbols, which are defined by the relation

$$
\partial_{\nu} \boldsymbol{b}_{\mu}=\frac{\partial^{2} \boldsymbol{r}}{\partial \xi^{\mu} \partial \xi^{\nu}}=: \Gamma_{\nu \mu}^{\lambda} \boldsymbol{b}_{\lambda}
$$


so that $d \boldsymbol{b}_{\mu}=\Gamma_{\nu \mu}^{\lambda} d \xi^{\nu} \boldsymbol{b}_{\lambda}$. The Christoffel symbols can be explicitly expressed via derivatives of the metric tensor (see any standard textbook on differential geometry, for example [Struik, 1988]). For numerical calculations, we will directly apply Equation(5.2) to calculate the $\Gamma_{\nu \mu}^{\lambda}$.

The Christoffel symbols will naturally appear in derivatives of vector fields $\boldsymbol{v}(\boldsymbol{r})=v^{\mu} \boldsymbol{b}_{\mu}$ (assuming, that the vector field is described by its contravariant components), because changes due to infinitesimal displacements $\boldsymbol{r} \rightarrow \boldsymbol{r}+d \boldsymbol{r}$ are not only due to changes in the vectors components but also due to the changing 3-bein:

$$
d \boldsymbol{v}(\boldsymbol{r})=\boldsymbol{v}(\boldsymbol{r}+d \boldsymbol{r})-\boldsymbol{v}(\boldsymbol{r})=\left[\left(\partial_{\nu} v^{\mu}\right)+\Gamma_{\nu \lambda}^{\mu} v^{\lambda}\right] d \xi^{\nu} \boldsymbol{b}_{\mu} .
$$

The expressions in square brackets form the components of a second rank tensor, called the covariant derivative

$$
D_{\nu} v^{\mu}:=\partial_{\nu} v^{\mu}+\Gamma_{\nu \lambda}^{\mu} v^{\lambda}
$$

Let us add two remarks:

(i) We can repeat the above steps assuming that the vector field is described by its covariant components, i.e. $\boldsymbol{v}=v_{\mu}^{\mu}$. The result is

$$
D_{\nu} v_{\mu}:=\partial_{\nu} v^{\mu}-\Gamma_{\nu \mu}^{\lambda} v_{\lambda}
$$

(ii) The concept can easily be generalised to higher rank tensor fields. For example, for a 2nd rank tensor field described by contravariant components $T^{\mu \nu}$ one finds

$$
D_{\mu} T^{\nu \lambda}=\left(\partial_{\mu} T^{\nu \lambda}+\Gamma_{\mu \rho}^{\nu} T^{\rho \lambda}+\Gamma_{\mu \rho}^{\lambda} T^{\nu \rho}\right)
$$

In particular one may check the important Ricci's Lemma, which states that the covariant derivative of the metric tensor vanishes identically, $D_{\mu} g^{\nu \lambda}=0$.

Now we can formulate derivatives of scalar and vector fields in general curvilinear coordinate systems. The change of a scalar field $f$ due to infinitesimal displacements defines the gradient operation of vector analysis:

$$
d f(\boldsymbol{r})=(\operatorname{grad} f) \cdot d \boldsymbol{r}=\left(\operatorname{grad}_{\mu} f\right) \cdot d \xi^{\mu},
$$

i.e. $\operatorname{grad}_{\mu} f=\partial_{\mu} f$. The gradient may as well be considered as the covariant derivative of a scalar field. The divergence is a linear differential operation on vector fields, which maps the vector field onto a scalar field. As we have seen above, differentiation maps vector fields onto second order tensor fields, from which a scalar can only be obtained by contraction. Thus the divergence operator must take on the form

$$
\operatorname{div} \boldsymbol{v}=D_{\mu} v^{\mu}
$$

The Laplace operator is the divergence of a gradient field, i.e. $\Delta f=\operatorname{div}(\operatorname{grad} f)=$ $D_{\mu}\left(g^{\mu \nu} \partial_{\nu} f\right)=g^{\mu \nu} D_{\mu} \partial_{\nu} f$, where we have made use of Ricci's Lemma in the last step. 
Consequently, the Laplacian will take on the following form in arbitrary (smooth) curvilinear coordinates:

$$
\Delta f(\boldsymbol{r})=\operatorname{div}(\operatorname{grad} f)=g^{\mu \nu}\left(\partial_{\mu} \partial_{\nu}-\Gamma_{\mu \nu}^{\lambda} \partial_{\lambda}\right)
$$

Now let us set up an algorithm to compute a discretized version of $\Delta$ up to second order accuracy and determine the corresponding hopping rates. For the differential operators applied to $\eta$, this is completely standard:

$$
\begin{gathered}
\partial_{\mu} \eta=\frac{1}{2 \Delta^{\mu}}\left(\eta\left(\xi^{\mu}+\Delta^{\mu}\right)-\eta\left(\xi^{\mu}-\Delta^{\mu}\right)\right)+O\left[\left(\Delta^{\mu}\right)^{2}\right] \\
\partial_{\mu} \partial_{\nu} \eta=\frac{1}{4 \Delta^{\mu} \Delta^{\nu}}\left[\eta\left(\xi^{\mu}+\Delta^{\mu}, \xi^{\nu}+\Delta^{\nu}\right)+\eta\left(\xi^{\mu}-\Delta^{\mu}, \xi^{\nu}-\Delta^{\nu}\right)+\right. \\
\left.-\eta\left(\xi^{\mu}+\Delta^{\mu}, \xi^{\nu}-\Delta^{\nu}\right)-\eta\left(\xi^{\mu}-\Delta^{\mu}, \xi^{\nu}+\Delta^{\nu}\right)\right] .
\end{gathered}
$$

Note that this form implies that hoppings to next nearest neighbours have to be included. What remains to be done is the numerical computation of the metric tensor and the Christoffel symbols. To compute $g^{\mu \nu}$ start from

$$
b_{\mu}^{i}=\partial_{\mu} x^{i}=\frac{1}{2 \Delta^{\mu}}\left(x^{i}\left(\xi^{\mu}+\Delta^{\mu}\right)-x^{i}\left(\xi^{\mu}-\Delta^{\mu}\right)\right)+\cdots
$$

and use

$$
g_{\mu \nu}=\sum_{i=1}^{3} b_{\mu}^{i} b_{\nu}^{i}
$$

The contravariant metric tensor has to be evaluated from

$$
g^{\mu \lambda} g_{\lambda \nu}=\delta_{\nu}^{\mu}
$$

To compute the Christoffel symbols start from Equation (5.2), which relates the $\Gamma_{\mu \nu}^{\lambda}$ to second order derivatives

$$
c_{\mu \nu}^{i}:=\partial_{\mu \nu} x^{i},
$$

which can be used in its discretized form. From Equation(5.2) we get by multiplying with $\boldsymbol{b}_{\kappa}$

$$
\sum_{i=1}^{3} c_{\mu \nu}^{i} b_{\kappa}^{i}=\Gamma_{\mu \nu}^{\lambda} \sum_{i=1}^{3} b_{\lambda}^{i} b_{\kappa}^{i}=g_{\kappa \lambda} \Gamma_{\mu \nu}^{\lambda}=: \Gamma_{\kappa \mu \nu}
$$

The quantities $\Gamma_{\kappa \mu \nu}$ are also called Christoffel symbols of the 2nd kind. Note that they are easily calculated numerically from the coefficients $b_{\mu}^{i}$ and $c_{\mu \nu}^{i}$. To obtain the $\Gamma_{\mu \nu}^{\kappa}$ we have to multiply with the contravariant metric tensor

$$
\Gamma_{\mu \nu}^{\lambda}=g^{\lambda \kappa} \Gamma_{\kappa \mu \nu}
$$


This completes the necessary algorithmic steps to assemble the Laplacian in arbitrary curvilinear coordinates, which we summarise in the recipe given on the next page. 


\section{Recipe for calculating hopping rates of TGMC}

1. Set up the system of grid points $\boldsymbol{r}(\xi)$ with $\boldsymbol{\xi}=k \Delta \xi_{1} \boldsymbol{e}_{1}+l \Delta \xi_{2} \boldsymbol{e}_{2}+m \Delta \xi_{3} \boldsymbol{e}_{3}$ Components will be denoted by

$$
x^{i}(k, l, m)
$$

2. Compute the Cartesian components of covariant base vectors

$$
b_{\mu}^{i}(k, l, m)=\partial_{\mu} x^{i}(k, l, m)
$$

by finite differencing to $2^{\text {nd }}$ order accuracy (central differences)

3. Compute the covariant metric tensor at every grid point

$$
g_{\mu \nu}(k, l, m)=\sum_{i} b_{\mu}^{i}(k, l, m) b_{\nu}^{i}(k, l, m)
$$

4. Compute the contravariant metric tensor $g^{\mu \nu}(k, l, m)$ by matrix inversion at every grid point

$$
g_{\mu \lambda}(k, l, m) g^{\lambda \nu}(k, l, m)=\delta_{\mu}^{\nu}
$$

5. Compute the components $c_{\mu \nu}^{i}(k, l, m):=\partial_{1}^{2} x^{i}(k, l, m)$ by finite differencing to $2^{\text {nd }}$ Order accuracy. Then compute the $\gamma^{\lambda}$ at every grid point

$$
\gamma^{\lambda}(k, l, m)=g^{\mu \nu}(k, l, m) g^{\lambda \kappa}(k, l, m) \sum_{i=1}^{3} c_{\mu \nu}^{i}(k, l, m) b_{\kappa}^{i}(k, l, m)
$$

6. Now set up the hopping rates according to the table given below.

\begin{tabular}{lr}
\hline Direction $\boldsymbol{a}$ & Hopping rate \\
\hline$\pm \Delta \xi_{\mu} \boldsymbol{e}_{\mu}$ & $\frac{g^{\mu \mu}(\boldsymbol{\xi})}{\left(\Delta \xi_{\mu}\right)^{2}}\left(1 \mp \frac{\gamma^{\mu}(\boldsymbol{\xi}) \Delta \xi_{\mu}}{2}\right)$ \\
$\pm \Delta \xi_{\mu} \boldsymbol{e}_{\boldsymbol{\mu}} \pm \Delta \xi_{\nu} \boldsymbol{e}_{\nu}$ & $\frac{g^{\mu \nu}(\boldsymbol{\xi})}{4 \Delta \xi_{\mu} \Delta \xi_{\nu}}$ \\
$\pm \Delta \xi_{\mu} \boldsymbol{e}_{\boldsymbol{\mu}} \mp \Delta \xi_{\nu} \boldsymbol{e}_{\nu}$ & $\frac{g^{\mu \nu}(\boldsymbol{\xi}+\boldsymbol{a})}{4 \Delta \xi_{\mu} \Delta \xi_{\nu}}$ \\
\hline
\end{tabular}

Hopping rates for the general curvilinear case, when moving from $\boldsymbol{r}(\boldsymbol{\xi})$ to $\boldsymbol{r}(\boldsymbol{\xi}+\boldsymbol{a})$. 


\subsection{Finite Volume Monte Carlo}

Finite volume methods are commonly used in numerical algorithms, which solve partial differential equations. They are particularly useful for equations of the form $\hat{L}(f)=g$, where the differential operator $\hat{L}$ is the divergence of something

$$
\hat{L}(f)=\operatorname{div} \cdot \hat{\boldsymbol{Q}}(f)
$$

The general idea is to discretise the system volume $\Omega$ into cells (called control volumes in this context) $\Omega_{k}$ with piecewise planar boundaries, such that two cells share a complete planar piece of surface or a complete common edge or a single vertex (or nothing at all). Then one uses Gauss's integral theorem on every control volume

$$
\int_{\Omega_{k}} \operatorname{div} \cdot \hat{\boldsymbol{Q}}(f)=\int_{\partial \Omega_{k}} \boldsymbol{\nu}_{k} \cdot \hat{\boldsymbol{Q}}(f) .
$$

$\left(\boldsymbol{\nu}_{k}\right.$ denotes the surface normal on $\partial \Omega_{k}$ ). As the boundary $\partial \Omega_{k}$ is composed of plane surfaces $P_{k, a}, a=1 \cdots n(k)$, the surface integral may be written as a sum

$$
\sum_{a=1}^{n(k)} \boldsymbol{n}_{a} \cdot \int_{P_{k, a}} \hat{\boldsymbol{Q}}(f)
$$

with a constant normal vector for every plane surface part. The original equation thus becomes a system

$$
\sum_{a=1}^{n(k)} \boldsymbol{n}_{a} \cdot \int_{P_{k, a}} \hat{\boldsymbol{Q}}(f)=\int_{\Omega_{k}} g
$$

and the integrals appearing in this system are approximated by numerical quadratures. If we represent every cell by a point $\boldsymbol{r}_{k}$ of its interior and look for numerical solutions $f\left(\boldsymbol{r}_{k}\right)$, the finite volume methods are called cell centred ${ }^{1}$ Note that this discretisation strategy implies that boundaries $\partial \Omega$, which do not consist of planar pieces can only be treated approximately. A boundary conform finite volume discretisation requires that the boundary is approximated by a mesh of planar surface parts, which correspond to planar surface parts of subvolumes.

In the following we will derive the hopping rates corresponding to finite volume dicretisations using simplices (i.e. tetrahedra in 3 dimensions and triangles in 2 dimensions) as subvolumes ${ }^{2}$. These shapes of the control volumes have important practical and conceptual advantages. To construct a tetrahedralisation we may start from an arbitrary set of points $\boldsymbol{x}_{m}$ (including boundary points on $\partial \Omega$ ) which shall become the vertices of

\footnotetext{
${ }^{1}$ In so called cell vertex methods, the unknown variables are put on the edges (vertices) of the control volumes.

${ }^{2}$ Such discretisations are also called tetrahedralisations of a volume in $3 \mathrm{~d}$ and triangulations of a surface in $2 \mathrm{~d}$
} 
the subvolume simplices. First, we set up auxiliary cells (which will not become the subvolumes) by the so called Voronoi-Dirichlet construction: A Voronoi cell $\omega_{m}$ contains all points, which are closer to $\boldsymbol{x}_{m}$ than to any other point of the prescribed set. Every $\omega_{m}$ is a convex set (if its boundary is included) with piecewise planar boundaries. The vertices $\boldsymbol{r}_{k}$ of these cells are called Voronoi points and they will become the representative points of the subvolume tetrahedra, which we are now going to construct. If several $\omega_{m}$ have a common Voronoi point on their boundaries, this point must have the same distance to all the corresponding $\boldsymbol{x}_{m}$ by construction, i.e. all these $\boldsymbol{x}_{m}$ have to fall on a sphere around the Voronoi point. For generic positions, only 4 points can fall on a common sphere. Exceptional cases (degenerate Voronoi points) with more than four $\boldsymbol{x}_{m}$ falling on a common sphere need not be considered here. They are easily removed by moving one of the $\boldsymbol{x}_{m}$ slightly. Thus cells, which are represented by Voronoi points $\boldsymbol{r}_{k}$ and have the $\boldsymbol{x}_{m}$ as vertices naturally become tetrahedra, which possess the so called Delaunay property: no $\boldsymbol{x}_{m}$ lies inside the circumsphere of each tetrahedron.

If all Voronoi points are regular, the emerging structure of Delaunay tetahedra is simplicial complex (i.e. consists of tetrahedra, sharing one or zero common surface triangles, a common single edge, a common single point or nothing), which constitutes a tetrahedralisation of $\Omega^{3}$, called Delaunay tetrahedralisation. It is the geometrical dual of the Voronoi tessellation: to every Voronoi cell there corresponds a Delaunay vertex, to every Voronoi edge there corresponds a Delaunay triangular surface part and to every Voronoi planar surface part there corresponds a Delaunay edge. Such tetrahedralisations have many interesting and very convenient properties. Efficient algorithms exist to construct such tetrahedral meshes, even if we require them to be boundary conform [Si, 2006]. Let us therefore start from a given, boundary conform (also called constrained Delaunay tetrahedralisation of $\Omega$ with tetrahedra $\omega_{k}$ and dual Voronoi points $\boldsymbol{r}_{k}$, which we choose as the points representing the subvolume of a Delaunay tetrahedron.

Following our general strategy we compare the discretized version of the continuum limit of the hopping Hamiltonian on the simplicial complex of Delaunay tretahedra with its original form such that we can simply read off the hopping rates. Let us consider a single tetrahedron (number $m$ ) together with its nearest neighbours $n=1 \cdots N(m)$. The hopping from $m \rightarrow n$ corresponds to a Hamiltonian

$$
\hat{H}_{m \rightarrow n}=-\sum_{n}^{N(m)} K(m \rightarrow n)\left(\varphi_{n}-\varphi_{m}\right) \eta_{m}
$$

and the corresponding subvolume integral is given by

$$
-D \int_{\Omega_{m}} d^{3} \boldsymbol{r} \eta(\boldsymbol{r}) \nabla^{2} \varphi(\boldsymbol{r})
$$

\footnotetext{
${ }^{3}$ Strictly speaking it is a tetrahedralisation of the convex hull of $\Omega$, but is easy to remove faces in a postprocessing step to conform with a non-convex boundary.
} 
Here we employ the finite volume discretisation described above

$$
-D \frac{\eta_{m}}{\left|\Omega_{m}\right|} \sum_{n}^{N(m)} \boldsymbol{\nu}_{m n} \cdot \int_{\partial \Omega_{m n}} \boldsymbol{\nabla} \varphi
$$

where $\partial \Omega_{m n}$ denotes the triangle shared by tetahedra $n$ and $m$ and $\boldsymbol{\nu}_{m n}$ are the corresponding normal unit vectors pointing outwards of tetrahedron $m$. We approximate the directional derivatives by finite differences using data at the representative points

$$
\nu_{n m} \cdot \nabla \varphi=\frac{\varphi_{n}-\varphi_{m}}{l_{n m}}+\cdots
$$

where $l_{m n}$ is the length of the link between $\boldsymbol{r}_{m}$ and $\boldsymbol{r}_{n}$. Inserting equation (5.6) into equation (5.5) and compare with equation(5.4) we can read off the hopping rate to neighbouring tetrahedra:

$$
K(m \rightarrow n)=D \frac{\left|\partial \Omega_{m n}\right|}{\left|\Omega_{m}\right| l_{m n}}
$$

where $\left|\partial \Omega_{m n}\right|$ is the area of the triangle $\partial \Omega_{m n}$ and $\left|\Omega_{m}\right|$ is the volume of tetrahedron $\mathrm{m}$. Thus the hopping rates are very easily constructed from the metric properties of the mesh, which can be determined by standard algorithms.

In many problems, the boundary of $\Omega$ is given as the only geometric information. Then a typical application of the FVMC consists of the following steps:

1. Determine a triangulation of the boundary surface

2. Determine a Delaunay tetrahedralisation which is boundary conform

3. Compute the metric property properties of this tetrahedralisation and set up the hopping rates according to equation $((5.7))$

For steps 1 and 2 we use the freely distributed TetGen and Qhull packages (see [Si, 2006] and [Barber et al., 1996] respectively). Furthermore we would like to remark that it is also possible to first apply a finite volume discretisation method to a simple cubic lattice. In this case the procedure is equivalent to a second order finite difference method. Thus, it is possible to combine finite volume with transformed grid methods. In fact, it is easily shown that the expressions for the hopping rate (5.7) after transforming to cylindrical and spherical coordinates gives the same rates as listed in the tables in section 5.2.1 and 5.2.2. Following this strategy, we may also apply different transformations to different parts of the cubic lattice and always keep consistent hopping rates across the boundaries of this region. 


\subsection{Eukaryotic Chemotaxis}

In the final section of this chapter, we now apply the new simulation method to show that the behaviour and outcome of signalling cascades depends on the underlying geometry of the reaction medium. To demonstrate this we will model the early steps in the chemotactic response of a single eukaryotic cell.

Chemotaxis refers to the directed movement of single cells towards a chemo-attractant or away from a chemo-repellent. It occurs both in prokaryotic as well as eukaryotic cells and it is an essential part in many biological processes. Morphogenesis and the guidance of immune cells to the site of infection are only two prominent examples, which depend on chemotaxis. For studying eukaryotic chemotaxis, the social amoebae Dictyostelium discoideum exposed to gradients of cyclic adenosine 3',5'-monophosphate (cAMP) has become the model system. Normally, Dictyostelium cells grow as single amoebae but when challenged with adverse environmental conditions, individual cells aggregate and form multicellular structures containing up to one million cells. In order to aggregate, the cells release cAMP to the environment and subsequently move towards that direction in which the CAMP concentration is high.

The chemotactic response in eukaryotic cells is generally subdivided into three phases [Devreotes and Janetopoulos, 2003]. In the first step which lasts around half a minute the cell senses the extracellular stimulus field. This step is known as gradient sensing. Polarisation constitutes the second step. During this stage the cell assumes a shape in which an axis with a well defined front and back is established. This step involves the recruitment of a multitude of different proteins to the plasma membrane and it takes place within 10 to 60 seconds after the application of the stimulus. During the last step the cell remodels its cytoskeleton which causes its movement.

\subsubsection{Gradient Sensing}

The ability of Dictyostelium cells to determine the direction of the concentration gradient is based on comparing the difference in receptor occupancy between the front and the back of the cell. The gradient sensing mechanism is very sensitive to extracellular cAMP concentration differences, i.e. it has been reported that Dictyostelium is capable of responding to a $2 \%$ difference in active receptors between the front and the back of the cell, when a total of only 400 receptors are bound to cAMP [Mato et al., 1975].

The most prominent feature of the gradient sensing signalling cascade is that it leads to the the rapid accumulation of phosphatidylinositol-(3,4,5)-triphosphate (PIP3) at the leading edge of the plasma membrane, i.e. at that part of the membrane which is exposed to the highest cAMP concentration. The accumulation of PIP3 to the front has been shown by monitoring the fluorescent signal emitted from GFP-tagged pleckstrin-homology 

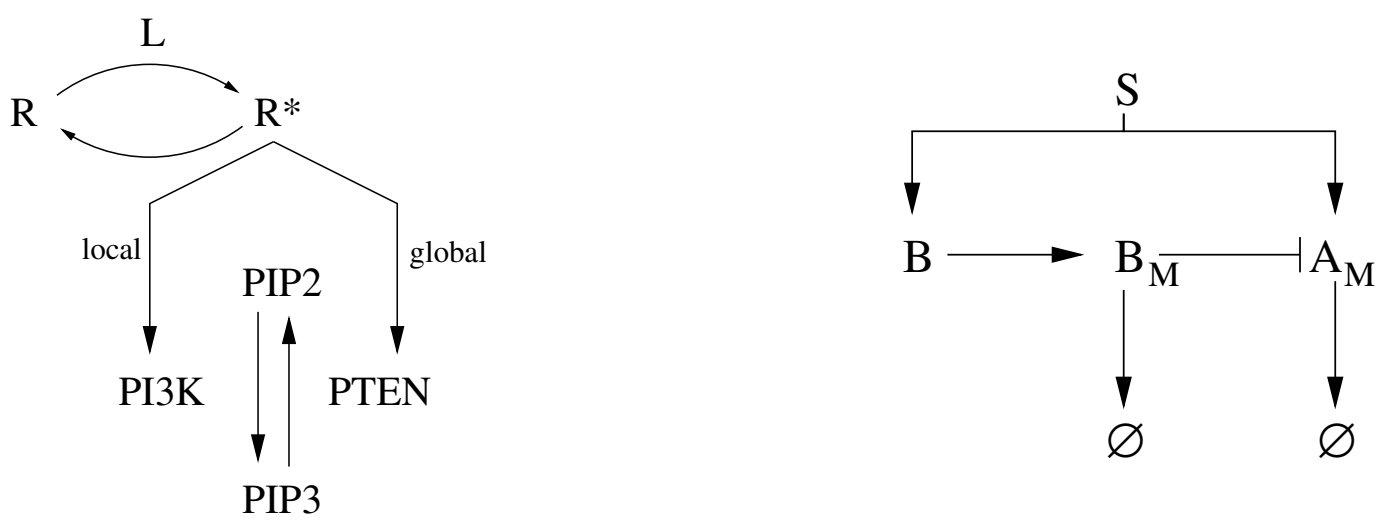

Figure 5.5: Left: General ratio of a local excitation global inhibition model as proposed by Levchenko and Iglesias [2002]. Right: The abstract model proposed by Levine et al. [2006].

$(\mathrm{PH})$ domain containing proteins which bind to PIP3. PIP3 itself is under the control of the kinase PI3K and the phosphatase PTEN. PI3K produces PIP3 by phosphorylating PIP2. As opposed to that PTEN has the antagonistic effect as it dephosphorylates PIP3 back to PIP2. Furthermore, it has been observed that PI3K localises to the front and PTEN localises to the back, if a cell is exposed to a gradient. However, in mutant studies in which the cells lack proper PI3K molecules, the direction sensing network is not impaired. Instead, only the speed of the movement is reduced [Postma et al., 2004]. Consequently, the PI3K/PTEN system can not be the primary and sole mechanism by which gradient information is processed. Instead, it has been proposed that small G proteins act as the initial activators for gradient sensing [Chung et al., 2001]. Overall, since the survival of Dictyostelium crucially depends on its chemotactic behaviour, it seems highly unlikely that the chemotactic response is mediated by a single molecule or a linear signalling network. Consequently the biochemical data will remain contradictory unless it is integrated in a larger picture.

Several models have been proposed so far for explaining gradient sensing [Devreotes and Janetopoulos, 2003]. Among these models are the so-called first hit inhibition and the intermediate depletion model. The first hit inhibition model is based on the idea of exploiting temporal differences in the arrival of the stimulus at the front and back of the cell when a gradient is applied. In particular this model assumes that inhibitory molecules are produced at the front and then rapidly diffuse to the back of the cell where they cause an inhibitory response. As opposed to that the intermediate depletion model assumes that a highly cooperative binding of some signalling molecules to some macromolecule at the front limits their availability at the back. Although both models seem reasonably plausible, they did not withstand experimental tests. The first hit inhibition model is for instance incapable of accounting for gradient reversal. Once a gradient has been estab- 
lished, it can not easily be reversed when the signal is changed. The inhibitory depletion model can not correctly account for the fact that a cell is responsive for a wide range of background stimulus concentrations.

Currently, the most promising approaches for modelling gradient sensing are based on the so-called local excitation global inhibition mechanism, because these models are capable of reproducing most of the experimental findings. According to this idea, activated receptors produce both an excitatory as well as an inhibitory signal. The gradient is then established because the activation signal only acts locally whereas the inhibitory signal is globally integrated. Consequently, excitation exceeds inhibition at the front of the cell whereas the opposite holds true for the back. Overall, this kind of model was first proposed by Levchenko and Iglesias [2002] and was subsequently extended in Ma et al. [2004]; but also the recently proposed model of Levine et al. [2006] belongs to this class. To compare both models, we have schematically visualised them in Figure 5.5. Obviously the model proposed by Levchenko and Iglesias [2002] tries to explain the uneven distribution of PIP molecules whereas the model of Levine et al. [2006] is more abstract as it does not explicitly link the involved constituents to real molecules. The chemical species in this model are simply denoted as $S, A$, and $B$ and account for a signal, an activator, and an inhibitor respectively. In general, an abstract model has the advantage that it does not have to postulate the existence of additional chemical species that have not been characterised in experiments. Moreover, in the model proposed by Levine et al. [2006] the inhibitor directly acts upon the activator and both molecules are annihilated when they react. As opposed to that, in the models of Levchenko and Iglesias [2002] and Ma et al. [2004] the activator (PI3K) and the inhibitor (PTEN) act on a third molecular species, which can either be in the active state (PIP3) or in the inactive state (PIP2). However, the two models are nevertheless based on the same idea that the excitation signal acts locally whereas the inhibitory signal is integrated over the entire cell. In particular this is accomplished by attaching the activator to the membrane whereas the inhibitor can freely diffuse in the cytosol.

In the next section we will based on the model of [Levine et al., 2006] devise a minimal abstract model of gradient sensing. However, before we do so, we would like to stretch that our model has to be understood as a conceptual model and that the chemical species which we use should not be confused with real chemical species such as PTEN or PI3K.

\subsubsection{Computational Modelling of Gradient Sensing}

As a start we reexamine the model proposed by [Levine et al., 2006] in somewhat greater detail. As the authors of that model offer an interpretation, which links the inhibitor and activator molecules to the different subunits of a G Protein, they distinguish between the inhibitor in the cytosol and the inhibitor bound to the membrane. However, the same behaviour of the model can be obtained when combining both species such that $I=B+B_{M}$. Consequently one arrives at the model depicted in the schematic represen- 

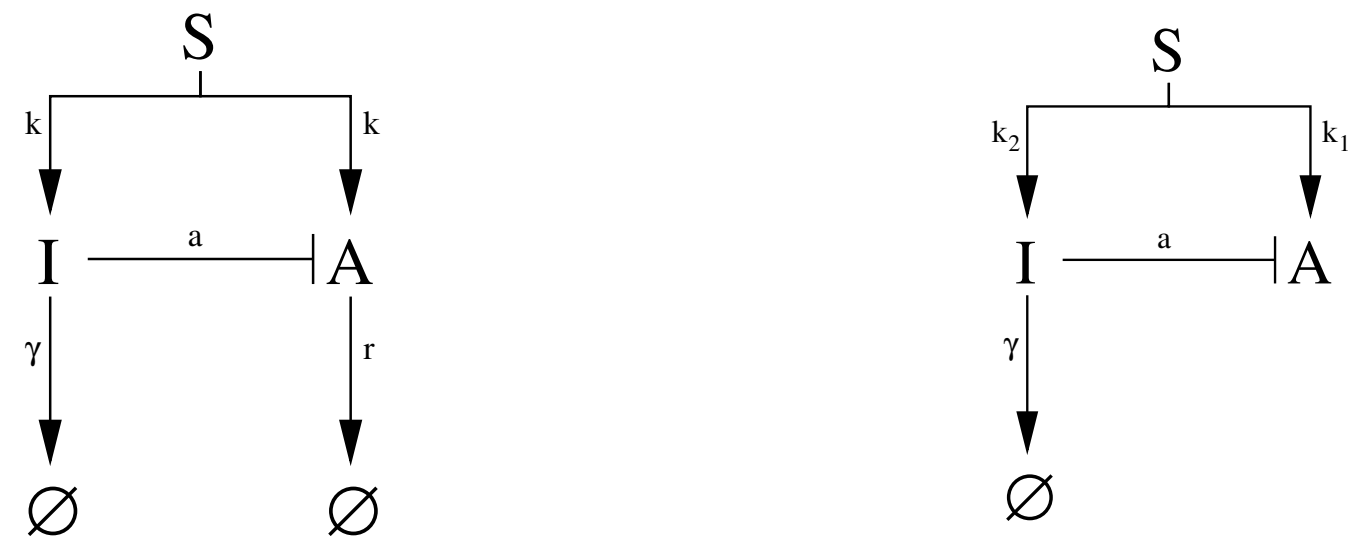

Figure 5.6: Schematic representation of the minimal model according to [Levine et al., 2006] (left) and our own model.

tation on the left of Figure 5.6. Since we require that annihilation reactions occur in this simplified model as often as in the original description, we have to reduce the value of the rate constant $a$ because the number of inhibitor molecules in the membrane is generally lower than the number of inhibitor molecules in the cytosol. The same argumentation holds true for the rate with which the inhibitor spontaneously decays. Hence we have to require that $\gamma<r$. However, a very small value for the spontaneous decay rate implies that the inhibitor concentration is spatially homogeneous for all admissible cell sizes because the average life time of an inhibitor molecule defined as $\tau=\gamma^{-1}$ becomes larger than the time it takes the molecule to cross the cell by diffusion. Moreover, the assumption made in the original model, that the inhibitor does not decay spontaneously, if it is localised in the cytosol seems very implausible from a physiological perspective. Apart from that, the response in the activator concentration in the simplified model as well as in the original model does not exhibit perfect adaptation. This can be seen, if one translates the schematic representation in Figure 5.6 into two chemical rate equations and subsequently solves for the homogeneous steady state concentration of the activator, one finds that this concentration depends on the signal concentration $S$.

In the following we will now change the simplified model of Levine et al. [2006], such that we obtain the model which is schematically displayed in the right part of Figure 5.6. In particular, we remove the spontaneous decay of the activator molecules in the membrane. The inhibitor molecules in the membrane still decay with rate $\gamma$. Up to now the signal produced both the activator and the inhibitor with equal rates. This will no longer be the case. Instead $\mathrm{A}$ is produced with rate $k_{1}$ and I with rate $k_{2}$. Finally, as in the model proposed by [Levine et al., 2006], A and I are both annihilated in the reaction $\mathrm{A}+\mathrm{I} \rightarrow \emptyset$. Overall the reaction scheme gives rise to the two chemical rate equations

$$
\partial_{t} \mathrm{~A}=k_{1} \mathrm{~S}-a \mathrm{~A} \mathbf{I}
$$


and

$$
\partial_{t} \mathbf{I}=k_{2} \mathrm{~S}-\gamma \mathrm{I}-a \mathrm{~A} \mathbf{I} .
$$

When analysing the homogeneous steady state, we find that the activator concentration is given by

$$
\overline{\mathrm{A}}=\frac{k_{1} \gamma}{a\left(k_{2}-k_{1}\right)} .
$$

Hence $A$ is independent of $S$ such that our mechanism exhibits perfect adaptation as claimed above. Moreover we also see that we have to require that $k_{2}>k_{1}$ in order to get meaningful results. Otherwise, in the limit of $k_{2} \rightarrow k_{1}$, one would approach a value of infinity for the steady state concentration of $A$. In fact, the difference between A and I would grow with the rate $\gamma I$, if both growth rates were identical. Overall, our approach of choosing different values for the rates with which the activator and the inhibitor molecules are produced is a pronounced difference to the original model proposed by [Levine et al., 2006]. In this model the rates had to be identical because the activator and inhibitor molecules were identified with the different subunits of a G-Protein. As opposed to that we do not offer such a specific physiological interpretation. Our model only claims that the pathway, which leads to the production of the inhibitor proceeds slightly faster speed the pathway leading to activation. Overall this is not an implausible assumption and has also been made by [Ma et al., 2004].

We start our computational analysis of the new model by first demonstrating that the system adapts perfectly, if it is exposed to a uniform stimulus. To show this, we distribute forty-five active receptors per $\mu \mathrm{m}^{2}$ on the plasma membrane of a cylindrical cell with a radius of $3.33 \mu \mathrm{m}$ and a height of $10 \mu \mathrm{m}$. After 60 seconds the stimulus is tripled. For simulating this system the remaining parameters are chosen as follows: $k_{1}=10 \mathrm{~s}^{-1}, k_{2}=10.05 \mathrm{~s}^{-1}, a=10 \mathrm{No}^{-1} \mu \mathrm{m}^{-3} \mathrm{~s}^{-1}, \gamma=0.2 \mathrm{~s}^{-1}, D_{I}=10 \mu \mathrm{m}^{2} \mathrm{~s}^{-1}$, and $D_{A}=0.1 \mu \mathrm{m}^{2} \mathrm{~s}^{-1}$. The results displayed in Figure 5.7 are obtained by averaging over twenty five trajectories and show the total number of activator molecules in the cell. At the beginning the system responds with a transient sharp peak because the cell initially contains neither activator nor inhibitor molecules. As the stimulus is tripled, the system again reacts with a transient peak in the activator concentration. However, the steady state value which the activator concentration assumes is identical with the concentration value after the first peak. Hence the steady state value for the activator concentration is independent of the applied signal and the system clearly exhibits perfect adaptivity.

Next we investigate, how the system responds, if it is exposed to a linear signal gradient. For this purpose we simulate the reaction system in a cylindrical cell with a height of $5 \mu \mathrm{m}$ and a diameter of $6.66 \mu \mathrm{m}$. The signal gradient starts with a density of 36.25 active receptors per $\mu m^{2}$ at the left boundary of the cell and subsequently rises to a value of 53.75 active receptors per $\mu m^{2}$ at the right boundary. The remaining parameters, i.e. the reaction and diffusion rates are chosen in the same way as above. For displaying the results we project the activator concentration onto the longitudinal axis of the cylinder 

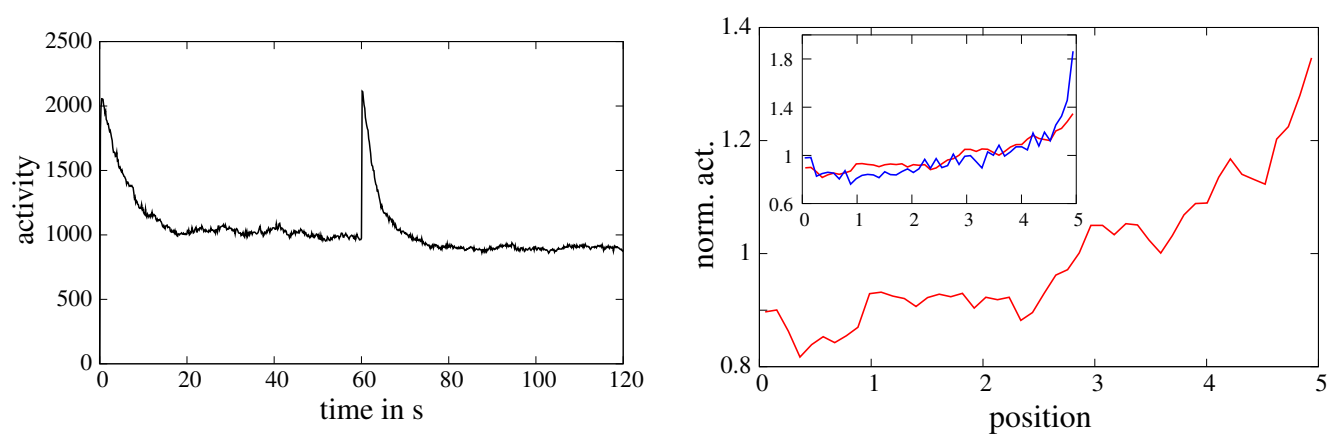

Figure 5.7: Left: The average activity is shown as a function of time. The system perfectly adapts when the stimulus is doubled at time $t=60$. Right: The main plot shows the normalised activator density along the cell in response to an signal gradient. The inset compares the response when using different rates for the annihilation reaction.

and divide by the average activator density. When averaging over an ensemble of two hundred profiles, we obtain the profile depicted in the main part of the right plot of Figure 5.7. Clearly the activator is distributed unevenly across the cell membrane. On the first $2.5 \mu \mathrm{m}$ the activator density stays almost constant. It then increases and exhibits a peak at the right boundary where the concentration of the signal is highest. Moreover we find that the build-up in the activator concentration becomes stronger the closer we get to the right boundary of the cell. Overall, the linear signal gradient is clearly mapped onto a nonlinear activator gradient in the cell membrane. Furthermore, we find that the strength of the peak at the right boundary depends on the rate of the annihilation reaction. When changing the reaction rate a from $10 \mathrm{No}^{-1} \mu \mathrm{m}^{-2} \mathrm{~s}^{-1}$ to $100 \mathrm{No}^{-1} \mu \mathrm{m}^{-2} \mathrm{~s}^{-1}$, we see an even larger increase of the activator concentration at the right end of the cell. This is visualised in the inset of the right part of Figure 5.7, in which the blue trajectory corresponds to the simulation results which are obtained with the high annihilation rate. In this case we clearly see that the activator exhibits a switching behaviour because it is predominantly locate in the right cap of the cell. However, by increasing the annihilation rate we also decrease the overall activator concentration such that fluctuations become large. In fact, on the level of a single profile it is almost impossible to observe the same behaviour as obtained by analysing the ensemble average. Overall this observation is in contrast to the finding of [Samadani et al., 2006], who showed that the distribution of the PIP3 concentration along the cell membrane is highly reproducible when performing several experiments in one and the same cell. Consequently the overall reaction network must contain a mechanism upstream of the initial gradient sensing mechanism, which effectively reduces the intrinsic noise. Finally, note that we have used very steep signal gradients. However, if we apply a much shallower signal gradient to the cell and average over an ensemble of two hundred simulations, we again find the same behaviour as above, i.e. the activator concentration shows a sharp increase at the leading edge of the cell. 

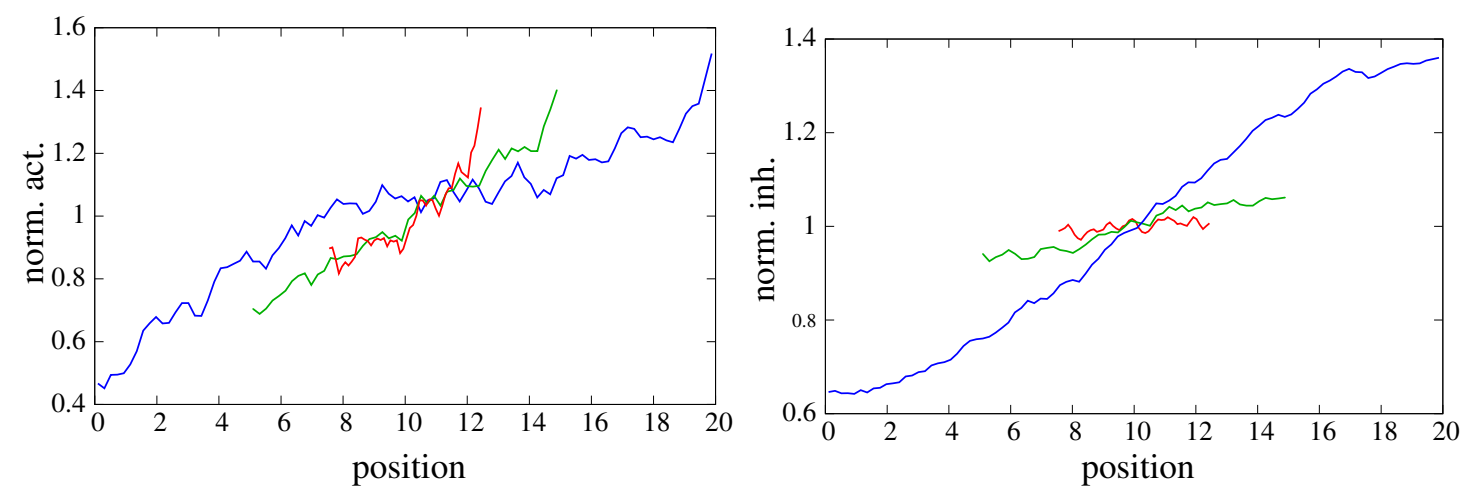

Figure 5.8: Effect of the underlying geometry on the gradient response. The left part shows the change of the normalised activator profile and the right part visualises how the inhibitor concentration changes.

In the following we will now analyse how the response of the system is altered, if we change the underlying reaction volume. In particular we will change the height of the cylindrical cell from 5 to 10 and $20 \mu \mathrm{m}$ while leaving the signal gradient unchanged. All three cells are exposed to the same gradient and since they all have the same centre of mass, the signal concentration in the middle of each cell will be identical. For analysing the results we again project the activator concentration onto the $z$-axis of the cylinder and divide by the average activator concentration. The ensemble averages of these profiles are shown in the left part of Figure 5.8. Obviously the distribution of the activator molecules is affected by the underlying geometry of the reaction medium. In particular we observe a transition from a profile with a sharp peak at the leading edge to a profile with a sigmoidal shape. Overall this change in the profile of the activator concentration goes along with a change in the distribution of the inhibitor molecules. This is visualised in the right part of Figure 5.8 in which the average profiles of the normalised inhibitor concentration are shown. Obviously the inhibitor concentration is constant in the small cell. As we increase the cell length this changes and ultimately we see a large difference in the inhibitor concentration between the left and the right boundary. Hence, in the large cell, the inhibitor concentration is no longer globally integrated. Instead, each point along the membrane only sees the inhibitor signal from its local neighbourhood. This behaviour emerges, if the size of the reaction medium exceeds the dispersion length of the inhibitor molecules. The dispersion length $l$ states how far a molecule diffuses before it decays, i.e. $l=\sqrt{D \gamma^{-1}}$. In the above system the dispersion length is approximately equal to $7 \mu \mathrm{m}$. Thus, it is no longer possible to abolish spatial inhomogeneities in the inhibitor concentration in the two larger cells by simple diffusion. Hence, the information about the strength of the signal at the leading edge can not be conveyed to the other end of the cell. Consequently, in the large cells the local excitation global inhibition mechanism breaks down such that we no longer observe the switch like behaviour in the activator 
concentration, which was observable in small cells.

\subsection{Discussion}

In this chapter we gave a detailed outline on how diffusion is modelled in a mesoscopic stochastic simulation environment. We have seen that the hopping rates for the Monte Carlo simulations are constructed in such way that diffusive motion is recovered on lengthscales that are large compared to the size of the subvolumes. In particular the exact expression for the hopping rates were obtained by comparing the discretized Laplacian with the hopping Hamiltonian. For representing the geometry of a reaction medium, we presented two different strategies inspired by the deterministic numerical treatment of partial differential equations. With the Transformed Grid Monte Carlo method one is capable of exactly mapping the boundary of the reaction medium on to a computational representation. As opposed to that the Finite Volume Monte Carlo method only approximates the boundary of a reaction medium as it fills the reaction medium with tetrahedra. However, the great advantage of the FVMC over the TGMC method is its greater flexibility. The underlying grid can easily be adapted and changed according to the state of the underlying reaction medium. In particular, we propose that the FVMC could be designed in such a way that the size of the gridcells follows the distribution of the reactivities meaning that a fine grid is automatically generated in those parts of the reaction medium at which the particle density and therefore the likelihood for the occurrence of reactions is high. For this purpose one can make use of already existing software and methods as the treatment of adaptive meshes is a common task in the deterministic numerical treatment of partial differential equations.

In the second part of this chapter we then applied our stochastic methods to simulate the chemical signalling cascade responsible for directional sensing in eukaryotic chemotaxis. In particular we have proposed a minimal reaction network for the gradient sensing network, which consist just of three chemical species. We showed that our model adapts perfectly to uniform stimuli. Moreover the system exhibits switching behaviour as the concentration of activator molecules is predominately increased at the leading edge, if the cell was exposed to a gradient stimulus. However, if the size of the reaction medium becomes larger than the dispersion length of the inhibitor molecules, the local excitation global inhibition mechanism breaks down, since the inhibitory signal can no longer be integrated over the entire cell. Note, that it should be possible to validate our predictions about the local excitation global inhibition model in experiments. For this one would have to deform the Dictyostelium cells mechanically, such that they become very large in a certain direction. The signal gradient can easily be control when using microfluidic devices. 


\section{Coda: Conclusion and outlook}

in which we simply come to an end.

6.1 Conclusion . . . . . . . . . . . . . . 101

6.2 Outlook . . . . . . . . . . . . . . . 102

\subsection{Conclusion}

Information processing in chemical signalling cascades is very complex as it involves the interplay of a multitude of different chemical species and reactions. Hence, modelling means that one has to focus on what one believes that is important and that one has to disregard the (unimportant) rest. Ultimately one wants to obtain a simplified description which captures the relevant aspects and clarifies how the system works. In particular, one wants to identify the critical features that explain the observed behaviour of a system. Furthermore, one wants to make predictions about the behaviour of the system by drawing simple logical conclusions. Overall these predictions should be such that they guide future experiments.

In order to model chemical signalling as outlined above, one needs to have the right tools at hand. Hence an integral part of this thesis focused on the development of such methods. In particular, we have argued throughout this thesis that one has to account for two features when dealing with chemical signalling cascades: On the one hand, it is important to correctly describe the noise that is inherently present in biological reaction systems and on the other one has to account for the geometry in which chemical signalling takes place.

In order to analytically quantify noise in chemical reaction networks we have introduced an operator description of the chemical master equation, which allows for the systematic description of all effects caused by noise in chemical reaction systems. Furthermore we have demonstrated the power of this approach by showing that noise may lead to the enhancement of a signal. When incorporating space into the modelling process, an analytical treatment becomes almost infeasible. Consequently one has to turn to stochastic computational methods. In this thesis we have therefore designed a new framework which allows for the stochastic simulation of reaction diffusion system in realistic geometries. 
For this purpose we have adopted two commonly used techniques for the integration of partial differential equations. Namely, we have designed the Transformed Grid Monte Carlo and the Finite Volume Monte Carlo method.

The TGMC technique was subsequently used for simulating a minimal model of the gradient sensing network in Dictyostelium discoideum. In particular our model showed perfect adaptation and mapped an external linear signal gradient onto an intracellular distribution of activator molecules, which showed a clear peak at the leading edge. Furthermore, we discussed the effects of changing the underlying geometry. In particular we have seen that the overall local excitation global inhibition mechanism requires that the reaction medium is smaller or of the same size as the dispersion length of the inhibitor molecules.

Furthermore, we have applied a stochastic simulation approach to study the effects of noise on the propagation of a signal by chemical wave fronts. In particular we have distinguished between intrinsic and spatial noise. We have seen that intrinsic noise in the bistable EGF receptor system leads to long tails in the underlying protein distributions. Consequently the system might spontaneously switch from one state to the other. Clearly, this behaviour can not be described by a purely deterministic reaction diffusion approach. Spatial noise is generally caused by molecular crowding. We have shown that our strategy of modelling this sort of noise by static obstacles is well in accordance with recent experimental work. Moreover, we have shown that a prototypical chemical wave front always propagates with constant velocity through an inhomogeneous medium. However, we also showed that the interface disperses, which ultimately limits the capabilities of this mechanism to propagate a signal over long distances.

\subsection{Outlook}

The work described in this thesis can be extended in multiple directions. In the near future we will apply the analytic methods presented in the second chapter to a variety of different motifs commonly encountered in chemical reaction networks. Overall we plan to construct a catalogue in which we list the effects that might arise due to noise in such building blocks of larger reaction networks.

Furthermore, it is planned to continue the work on the chemotactic response in Dictyostelium. Especially the formation of pseudopods provides an interesting and challenging problem. It has been suggested that the formation of these structures is linked to localised spots of signalling activity on the membrane [Postma et al., 2003]. With the help of the existing simulation routines one could analyse how the size, shape, and stability of these spots is affected by the underlying geometry. Furthermore it is planned to extend the Finite Volume Monte Carlo Method in such a way that it can account for a change in the underlying reaction medium during the simulation. That way one would be able to model the growth of pseudopods. 


\section{Bibliography}

S. M. Ajay and U. S. Bhalla. Synaptic plasticity in vitro and in silico: Insights into an intracellular signaling maze. Physiology, 21:289-296, 2006.

B. Alberts, D. Bray, J. Lewis, M. Raff, K. Roberts, and J. D. Watson. Molcular Biology of the Cell. Garland, 1994.

R. Alves, F. Antunes, and A. Salvador. Tools for kinetic modelling of biochemical networks. Nat. Biotechnol., 24:667-672, 2006.

M. Ander, P. Beltrao, B. Di Ventura, J. Ferkingoff-Borg, M. Foglierini, A. Kaplan, C. Lemerle, I.Tomas-Oliveira, and L. Serrano. SmartCell, a framework to simulate cellular processes that combines stochastic approximation with diffusion and localisation: analysis of simple networks. Syst. Biol., 1:129-138, 2004.

S.S. Andrews and D. Bray. Stochastic simulation of chemical reactions with spatial resolution and single molecular detail. Phys. Biol., 1:137-15, 2004.

A. Arkin, J. Ross, and H.H. McAdams. Stochastic kinetic analysis of developmental pathway bifurcation in phage $\lambda$-infected Escherichia coli cells. Genetics, 149:814-819, 1998.

C.B. Barber, D.P. Dobkin, and H.T. Huhdanpaa. The Quickhull algorithm for convex hulls. ACM Tran. Math. Softw., 22:469-483, 1996.

E. Bettelheim and B. Lehmann. Microscopic simulation of reaction-diffusion processes and applications of population biology and product marketing. In D. Stauffer, editor, Annual Reviews of Computational Physics VII. World Scientific, Singapore, 1999.

U.S. Bhalla. Signaling in small subcellular volumes. I. Stochastic and diffusion effects on individual pathways. Biophys. J., 87:733-744, 2004.

J. Blom and M. Peletier. The importance of being cigar-shaped. Technical report, Centrum voor Wiskunde en Informatica, 2002.

Y. Cao, H. Li, and L. Petzold. Efficient formulation of the stochastic simulation algorithm for chemically reacting systems. J. Chem. Phys., 121:4059-4067, 2004.

J. Cardy and U. Täuber. Field theory of branching and annihilating random walks. J. Stat. Phys., 90:1-56, 1998. 
C. Y. Chung, S. Funamoto, and R. A. Firtel. Signaling pathways controlling cell polarity and chemotaxis. Trends Biochem. Sci., 26:557-566, 2001.

P. Devreotes and C. Janetopoulos. Eukaryotic Chemotaixs: Distinctions between directional sensing and polarization. J. Biol. Chem., 278:20445-20448, 2003.

M. Doi. Stochastic theory of diffusion-controlled reaction. J. Phys. A: Math. Gen., 9: 1479-1495, 1976.

J. Elf and M. Ehrenberg. Spontaneous separation of bi-stable biochemical systems into spatial domains of opposite phase. Syst. Biol., 1:230-236, 2004.

R. John Ellis. Macromolecular crowding: obvious but underappreciated. Trends Biochem. Sci., 26:597-604, 2001.

M.B. Elowitz, M.G. Surette, P.E. Wolf, J.B. Stock, and S. Leibler. Protein mobility in the cytoplasm of Escherichia coli. J. Bacteriol., 181:197-203, 1999.

M.B. Elowitz, A.J. Levine, E.D. Siggia, and P.S. Swain. Stochastic gene expression in a single cell. Science, 297:1183-1186, 2002.

D. Fange and J. Elf. Noise-induced min phenotypes in E. choli. PLOS Comp. Biol., 2: 637-648, 2006.

A.M. Ferrenberg, D.P. Landau, and Y.J. Wong. Monte Carlo Simulations: Hidden errors from good random number generators. PRL, 69:3382-3384, 1992.

K. Fichthorn and W. Weinberg. Theoretical foundations of dynamical Monte Carlo simulations. J. Chem. Phys., 95:1090-1096, 1991.

T. Fricke and D. Wendt. The Markoff-Automaton A New Algorithm for Simulating the Time-Evolution of Large Stochastic Dynamic Systems. Int. J. Mod. Phys. C, 6: 277-306, 1995.

L. Gammaitoni, P. Hänggi, Peter Jung, and Fabio Marchesoni. Stochastic resonance. Rev. Mod. Phys., 70:223-287, 1998.

J. Garcia-Ojalvo, J. Sancho, and L. Ramirez-Piscena. A non-equilibrium phase transition with colored noise. Phys. Lett. A, 46:4670, 1992.

M.A. Gibson and J. Bruck. Efficient Exact Stochastic Simulation of Chemical Systems with Many Species and Many Channels. J. Phy. Chem., 104:1876-1889, 2000.

D. T. Gillespie. Approximate accelerated stochastic simulation of chemically reacting systems. J. Chem. Phys., 115:1716-1733, 2001. 
D. T. Gillespie. A General Method for Numerically Simulating the Stochastic Time Evolution of Coupled Chemical Reactions. Journal of Computational Physics, 22:403434, 1976.

D. T. Gillespie. Exact stochastic simulation of coupled chemical reactions. J. Phys. Chem., 81:2340-2361, 1977.

D. D. Ginty and R. A. Segal. Retrograde neurotrophin signaling: Trk-ing along the axon. Curr. Opin. Neurobiol., 12:268-274, 2002.

H. Grabert. Projection operator techniques in nonequilibrium statistical mechanics, volume 95 of Springer Tracts in Modern Physics. Springer, 1982.

J. Hattne, D. Fange, and J. Elf. Stochastic reaction-diffusion simulation with MesoRD. Bioinformatics, 21:2923-2924, 2005.

J. Hoschek and D. Lasser. Grundlagen der geometrischen Datenverarbeitung. B.G. Teubner, 1992.

C. Janetopoulos, L. Ma, P. N. Devreotes, and Pablo A. Iglesias. Chemoattractant-induced phosphatidylinositol 3,4,5-triphosphate accumulation is spatially amplified and adapts, independent of the actin cytoskeleton. PNAS, 101:8951-8956, 2004.

B. Kholodenko, G. Brown, and J. Hoek. Diffusion control of protein phosphorylation in signal transduction pathways. Biochem. J., 350:901-907, 2000.

B. N. Kholodenko. Cell-signalling dynamics in time and space. Nat. Rev. Mol. Cell. Bio., 7:165-176, 2006.

M. Kirschner. The meaning of systems biology. Cell, 121:503-504, 2005.

H. Kleinert. Pfadintegrale. B.I. Wissenschaftsverlag, 1993.

P. Knabner and L. Angermann. Numerik partieller Differentialgleichungen. SpringerVerlay, 2000.

R. Kopelman. Fractal Reaction Kinetics. Science, 241:1620-1626, 1988.

L.D. Landau and J. M. Lifschitz. Lehrbuch der theoretischen Physik (Band 1) Mechanik. Harri-Deutsch Verlag, 1997.

K.W. Lee, S.E. Webb, and A.L. Miller. A wave of free cytosolic calcium traverses zebrafish eggs on activation. Dev. Biol., 214:168, 1999.

C. Lemerle, B. Di Ventura, and L. Serrano. Space as the final frontier in stochastic simulations of biological systems. FEBS Lett., 579, 2005. 
Andre Levchenko and Pablo A. Iglesias. Models of eukaryotic gradient sensing: Application to chemotaxis of amoebae adn neutrophils. Biophys. J., 82:50-63, 2002.

H. Levine, I. Aranson, L. Tsimring, and T.V. Truong. Genetic feedback governs cAMP spiral wave formation in Dictyostelium. Proc. Natl. Acad. Sci. USA, 93:6382, 1996.

H. Levine, D. A. Kessler, and W. Rappel. Directional sensing in eukaryotic chemotaxis: A balanced inactivation model. Proc. Natl. Acad. Sci. USA, 103:9761-9766, 2006.

K. Lipkow, S.S. Andrews, and D. Bray. Simulated diffusion of phosphorylated CheY through the cytoplasm of Escherichia coli. J. Bacteriol., 187:45-53, 2005.

L. Lok. The need for speed in stochastic simulation. Nat. Biotechnol., 22:964-965, 2004.

L. Ma, C. Janetoppoulos, L. Yang, P. N. Devreotes, and P. A. Iglesias. Two complementary, local excitation, global inhibition mechanisms acting in parallel can explain the chemoattractant-induced regulation of $\mathrm{PI}(3,4,5) \mathrm{P}_{3}$ response in Dictyostelium cells. Biophys. J., 87:3764-3774, 2004.

B.L. Macinnis and R.B. Campenot. Retrograde support of neuronal survival without retrograde transport of nerve growth factor. Science, 295:1536-1539, 2002.

J.M. Mato, V. Nanjundiah, and T.M. Konijn. Signal input for a chemotactic response on the cellular slime mold Dictyostelium discoideum. PNAS, 72:4991-4993, 1975.

N. Metropolis, A. Rosenbluth, M. Rosenbluth, A. Teller, and E. Teller. Equation of state calculations by fast computing machines. J. of Chem. Phys., 21:1087-1092, 1953.

I. I. Moraru and L. M. Loew. Intracellular signaling: Spatial and temporal control. Physiology, 20:169-179, 2005.

C. Morton-Firth and D. Bray. Predicting temporal fluctuations in an intracellular signaling pathway. J. Theor. Biol., 192:117-128, 1998.

L. O. Murphy, S. Smith, R. H. Chen, D. C. Fingar, and J.Blenis. Molecular interpretation of ERK signal duration by immediate early gene products. Nat. Cell Biol., 4:556-564, 2002.

J. D. Murray. Mathematical Biology. Springer Verlag, Berlin, 1993.

A. Östman and F. D. Böhmer. Regulation of tyrosine kinase signalling by protein tyrosine phosphatases. Trends Cell Biol., 11:258-266, 2001.

E. M. Ozbudak, M. Thattai, I. Kurtser, A. D. Grossman, and A. van Oudenaarden. Regulation of noise in the expression of a single gene. Nat. Genet., 31:258-266, 2002. 
L. Peliti. Some observations on kinetic walk models. J. physique, Lett., 45:925-928, 1984.

N.V. Popov. Functional integrals and collective excitations. Cambridge University Press, 1981.

M. Postma, J. Roelofs, J. Goedhart, T. W. J. Gadella, A. Visser, and P. J. M. Van Haastert. Uniform cAMP stimulation of Dictyostelium cells induces localized patches of signal transduction and pseudopodia. Mol. Biol. Cell, 14:5019-5027, 2003.

M. Postma, L. Bosgraaf, H. M. Loovers, and P. J.M. Van Haastert. Chemotaxis: signalling modules join hands at front and tail. EMBO Rep., 5:35-40, 2004.

W. H. Press, S. A. Teukolsky, W. T. Vetterling, and B. P. Flannery. Numerical Recipes in $\mathrm{C}++$ - The Art of Scientific Computing. Cambridge University Press, Cambridge, 2002.

C. V. Rao, D. M. Wolf, and A. P. Arkin. Control, exploitation and tolerance of intracellular noise. Nature, 420:231-237, 2002.

A. R. Reynolds, C. Tischer, P. J. Verveer, O. Rocks, and P. I. H. Bastiaens. EGFR activation coupled to inhibtion of tyrosine phosphatases causes lateral signal propagation. Nat. Cell Biol., 5:447-453, 2003.

J. Riordan, C. R. Doering, and D. ben-Avraham. Fluctuations and stability of Fisher waves. Phys. Rev. Lett., 75:565-568, 1995.

A. Samadani, J. Mettetal, and A. van Oudenaarden. Cellular asymmetry and individuality in directional sensing. PNAS, 103:11549-11554, 2006.

J.M. Sancho and J. Garcia-Ojalvo. Noise induced order in extended systems: a tutorial. In J. Freund and T. Pöschl, editors, Stochastic processes in physics, chemistry and biology, volume 557 of Lecture notes in physics. Springer, 2000.

M.A. Santos and J.M. Sancho. Noise induced fronts. Phys. Rev. E, 59:98-102, 1999.

A. Sawano, S. Takayama, M. Matsuda, and A. Miyawaki. Lateral propagation of EGF signaling after local stimulation is dependent on receptor density. Dev. Cell., 3:245-257, 2002.

M. J. Saxton. Anomalous Diffusion due to obstacles: a Monte Carlo study. Biophys. J., 66:394-401, 1994.

J. Schnakenberg. Algorithmen in der Quantenmechanik und Statistischen Physik. Zimmermann-Neufang, Ulmen, 1995. 
P. Schwille, U. Haupts, S. Maiti, and W. W. Webb. Molecular dynamics in living cells observed by fluorescence correlation spectroscopy with one- and two-photon excitation. Biophys. J., 77:2251-2265, 1999.

R. Sedgwick and Christopher J. Van Wyk. Algorithms in C++. Addison-Wesley, Reading, Massachusetts, 2002.

N. M. Shnerb, Y. Louzoun, E. Bettelheim, and S. Solomon. The importance of being discrete - life always wins on the surface. PNAS, 97:10322-10324, 2003.

Hang Si. Tetgen, 2006. URL http://tetgen.berlios.de.

V. Sourjik. Receptor clustering and signal processing in E. coli chemotaxis. Trends Microbiol., 12:569-576, 2004.

J. R. Stiles and T. M. Bartol. Monte Carlo Methods for Simulating Realistic Synaptic Microphysiology Using Mcell. In Erik De Schutter, editor, Computational Neuroscience: Realistic Modeling for Experimentalists, pages 87-127. CRC Press, 2001.

D. J. Struik. Lectures on classical differential geometry. Dover, 1988.

A. Stundzia and C. Lumsden. Stochastic simulation of coupled reaction-diffusion processes. J. Comp. Phys., 127:196-207, 1996.

M. Thattai and A. van Oudenaarden. Intrinsic noise in gene regulatory networks. PNAS, 98:8614-8619, 2001.

C. Tischer. Nanoscale imaging of restricted cell membrane receptor diffusion. PhD thesis, Humboldt-Universität Berlin, 2005.

J. J. Tyson, K. C. Chen, and B. Novak. Sniffers, buzzers, toggles and blinkers: dynamics of regulatory and signaling pathways in the cell. Curr Opin.Cell Biol., 15:221-231, 2003.

P. J. Verveer, F S. Wouters, A. R. Reynolds, and P. I. H. Bastiaens. Quantitative imaging of lateral ErbB1 receptor signal propagation in the plasma membrane. Science, 290: 1567-1570, 2000.

H. Steven Wiley, Stanislav Y. Shvartsman, and Douglas A. Lauffenburger. Computational modelling of the EGF-receptor system: a paradigm for systems biology. Trends Cell Biol., 13:43-50, 2003. 


\section{Acknowledgements}

First of all my thanks go to Prof. Reiner Kree for giving me the freedom to pursue my ideas. He has been a motivating and inspiring mentor over these past years. I would also like to thank Prof. Annette Zippelius for her interest and support in my work. I am grateful to Mukund Thattai for giving me the opportunity to expand my horizons. My stay at NCBS in Bangalore from January to April 2006 has been a most memorable time. Furthermore I would like to thank Gisela Mesecke and the other administrative assistants for their constant support and for reducing my bureaucratic load to a minimum.

The life at this institute would not have been half as enjoyable without my colleagues and friends Christian, Timo, Axel, Peter, Martin, Bernd and all the other people past and present. Special thanks go to Christian who was sufficiently wacko to share an office with me for more than three years. At this point, I would also like to apologise to Timo that he always had to cope with my cunning plans. As a matter of fact, most of these ideas eventually turned out to be what they were in the first place, just plain bockmist.

Finally I would like to thank all those decomposing composers, who with their music made my daily life more enjoyable. I wish I had their talent, but let's face it, the relationship between a mediocre hornist doing computational biology at a physics institute and a composer is perfectly summarised by the following lines of Wilhelm Busch:

Wenn einer, der mit Mühe kaum

Gekrochen ist auf einen Baum,

Schon meint, daß er ein Vogel wär,

So irrt sich der. 



\section{Curriculum vitae}

Born:

Parents:

Country of Citizenship:
January 26, 1978 in Henstedt-Ulzburg, Germany Heike and Gerfried Jentsch

Germany

\section{Education}

2006

Scientific visist to Mukund Thattai National Center for Biological Sciences, Bangalore, India

2003

Master of Science in Neuroscience University Göttingen

2001

Bachelor of Science in Cognitive Science University Osnabrück, Germany

$2000 / 2001$

Continuation of Studies in Gothemburg University Gothemburg, Sweden

1998

Abitur

Dahlmannschule Bad Segeberg, Germany 